\title{
MINIMAL MODELS AND ABUNDANCE FOR POSITIVE CHARACTERISTIC LOG SURFACES
}

\author{
HIROMU TANAKA
}

\begin{abstract}
We discuss the birational geometry of singular surfaces in positive characteristic. More precisely, we establish the minimal model program and the abundance theorem for $\mathbb{Q}$-factorial surfaces and for log canonical surfaces. Moreover, in the case where the base field is the algebraic closure of a finite field, we obtain the same results under much weaker assumptions.
\end{abstract}

\section{Contents}

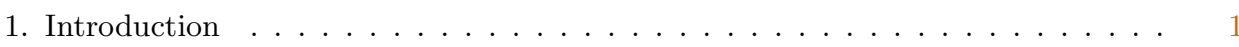

2. Notation and known results ...................... 6

3. $\mathbb{Q}$-factorial surfaces ......................... . . . . . 7

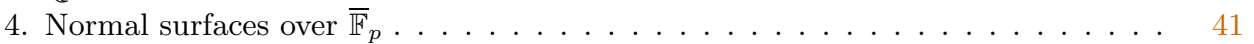

5. Log canonical surfaces . . . . . . . . . . . . . . . . . . . . . . . . . . . 49

6. Relativization . . . . . . . . . . . . . . . . . . . . . 55

Appendix A. Base-point-free theorem . . . . . . . . . . . . . . . 62

Appendix B. Rational singularities . . . . . . . . . . . . . . . . . . . 65

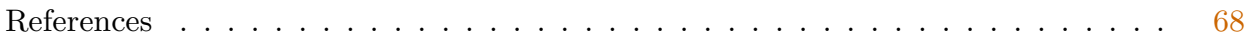

\section{$\S 1$. Introduction}

In this paper, we consider the minimal model theory for surfaces with some singularities in positive characteristic. If the singularities are $\mathbb{Q}$-factorial or $\log$ canonical, then we establish the minimal model program and the abundance theorem. In the case where the base field is the algebraic closure of a finite field, we obtain the same result under much weaker assumptions. More precisely, we prove the following two theorems in this paper.

Theorem 1.1 (Minimal model program). Let $X$ be a projective normal surface $X$, which is defined over an algebraically closed field $k$ of positive characteristic. Let $\Delta$ be an $\mathbb{R}$-divisor on $X$, and let $\Delta=\sum_{j \in J} \delta_{j} \Delta_{j}$ be its prime decomposition. Assume that one of the following conditions holds:

Received November 17, 2012. Revised June 9, 2013. Accepted August 18, 2013.

First published online September 9, 2014.

2010 Mathematics Subject Classification. Primary 14E30; Secondary $14 J 10$.

(C) 2014 by The Editorial Board of the Nagoya Mathematical Journal 
(QF) $X$ is $\mathbb{Q}$-factorial, and $0 \leq \delta_{j} \leq 1$ for all $j \in J$;

(FP) $k=\overline{\mathbb{F}}_{p}$, and $0 \leq \delta_{j}$ for all $j \in J$;

(LC) $(X, \Delta)$ is a log canonical surface.

Then, there exists a sequence of birational morphisms

$$
(X, \Delta)=:\left(X_{0}, \Delta_{0}\right) \stackrel{\phi_{0}}{\rightarrow}\left(X_{1}, \Delta_{1}\right) \stackrel{\phi_{1}}{\rightarrow} \cdots \stackrel{\phi_{s-1}}{\rightarrow}\left(X_{s}, \Delta_{s}\right)=:\left(X^{\dagger}, \Delta^{\dagger}\right)
$$

where $\left(\phi_{i-1}\right)_{*}\left(\Delta_{i-1}\right)=: \Delta_{i}$, with the following properties.

(1) Each $X_{i}$ is a projective normal surface.

(2) Each $\left(X_{i}, \Delta_{i}\right)$ satisfies $(Q F)$, (FP), or (LC) according to the above assumption.

(3) For each $i, \operatorname{Ex}\left(\phi_{i}\right)=: C_{i}$ is a proper irreducible curve such that

$$
\left(K_{X_{i}}+\Delta_{i}\right) \cdot C_{i}<0
$$

and such that $C_{i}$ generates an extremal ray.

(4) The pair $\left(X^{\dagger}, \Delta^{\dagger}\right)$ satisfies one of the following conditions:

(a) $K_{X^{\dagger}}+\Delta^{\dagger}$ is nef;

(b) there is a surjective morphism $\mu: X^{\dagger} \rightarrow Z$ to a smooth projective curve $Z$ such that $\mu_{*} \mathcal{O}_{X^{\dagger}}=\mathcal{O}_{Z},-\left(K_{X^{\dagger}}+\Delta^{\dagger}\right)$ is $\mu$-ample, and $\rho\left(X^{\dagger} / Z\right)=1$;

(c) $-\left(K_{X^{\dagger}}+\Delta^{\dagger}\right)$ is ample, and $\rho\left(X^{\dagger}\right)=1$.

In case (a), we say that $\left(X^{\dagger}, \Delta^{\dagger}\right)$ is a minimal model of $(X, \Delta)$.

In cases (b) and (c), we say that $\left(X^{\dagger}, \Delta^{\dagger}\right)$ is a Mori fiber space.

THEOREM 1.2 (Abundance theorem). Let $X$ be a projective normal surface $X$, which is defined over an algebraically closed field $k$ of positive characteristic. Let $\Delta$ be an $\mathbb{R}$-divisor on $X$, and let $\Delta=\sum_{j \in J} \delta_{j} \Delta_{j}$ be its prime decomposition. Assume that one of the following conditions holds:

(QF) $X$ is $\mathbb{Q}$-factorial, and $0 \leq \delta_{j} \leq 1$ for all $j \in J$;

(FP) $k=\overline{\mathbb{F}}_{p}$, and $0 \leq \delta_{j}$ for all $j \in J$;

(LC) $(X, \Delta)$ is a log canonical surface.

If $K_{X}+\Delta$ is nef, then $K_{X}+\Delta$ is semiample.

Note that, if $X$ is a normal surface over $\overline{\mathbb{F}}_{p}$, then $X$ is $\mathbb{Q}$-factorial (see Theorem 4.5). In particular, $K_{X}+\Delta$ is an $\mathbb{R}$-Cartier $\mathbb{R}$-divisor.

In the case where the characteristic of the base field is 0 , the above two theorems are proved by Fujino [F2]. His proofs heavily depend on the 
Kodaira vanishing theorem and its generalizations. Unfortunately, in positive characteristic, counterexamples to the Kodaira vanishing theorem exist (see $[R]$ ). To prove the above two theorems, we use a result established in $[\mathrm{Kel}]$.

In characteristic 0, the base-point-free theorem follows from the Kawamata-Viehweg vanishing theorem, which is a generalization of the Kodaira vanishing theorem (see [KoM, Theorem 3.3]). Although we cannot use the Kodaira vanishing theorem, we can show the following base-point-free theorem.

TheOREM 1.3 (Base-point-free theorem). Let $X$ be a projective normal $\mathbb{Q}$-factorial surface $X$, which is defined over an algebraically closed field $k$ of positive characteristic. Let $\Delta$ be a $\mathbb{Q}$-divisor. Let $\Delta=\sum_{j \in J} \delta_{j} \Delta_{j}$ be its prime decomposition, and assume that $0 \leq \delta_{j}<1$ for all $j \in J$. Let $D$ be a nef Cartier divisor satisfying one of the following properties:

(1) $D-\left(K_{X}+\Delta\right)$ is nef and big;

(2) $D-\left(K_{X}+\Delta\right)$ is semiample.

Then $D$ is semiample.

Although counterexamples to the Kodaira vanishing theorem exist, we can use the relative Kawamata-Viehweg vanishing theorem for birational morphisms of surfaces [KoK, Section 2.2]. Then, we obtain the following result on rational singularities.

THEOREM 1.4. Let $X$ be a projective normal surface $X$, which is defined over an algebraically closed field $k$ of positive characteristic. Let $\Delta$ be an $\mathbb{R}$-divisor on $X$. Let $\Delta=\sum_{j \in J} \delta_{j} \Delta_{j}$ be its prime decomposition, and assume that $0 \leq \delta_{j} \leq 1$ for all $j \in J$. Assume that $X$ has at worst rational singularities. Then, the following assertions hold.

(1) The surface $X$ is $\mathbb{Q}$-factorial. In particular, by Theorem 1.1, we can run a $\left(K_{X}+\Delta\right)$-minimal model program:

$$
\begin{aligned}
& (X, \Delta)=:\left(X_{0}, \Delta_{0}\right) \stackrel{\phi_{0}}{\rightarrow}\left(X_{1}, \Delta_{1}\right) \stackrel{\phi_{1}}{\rightarrow} \cdots \stackrel{\phi_{s-1}}{\rightarrow}\left(X_{s}, \Delta_{s}\right), \\
& \text { where }\left(\phi_{i-1}\right)_{*}\left(\Delta_{i-1}\right)=: \Delta_{i} .
\end{aligned}
$$

(2) Each $X_{i}$ has at worst rational singularities.

\subsection{Overview of related literature}

We summarize some literature related to this paper with respect to the surface theory, the minimal model theory, and Keel's result (Theorem 2.2). 
1.1.1. Surface theory. The Italian school established the classification theory for smooth algebraic surfaces, which was generalized by Kodaira, Shafarevich's seminar, and Bombieri and Mumford ([BM1], [BM2], [Mu2]). Theories of log surfaces and normal surfaces have been developed by Iitaka, Kawamata, Miyanishi, Sakai, and many others (see, e.g., [S], [Mi]). Fujita [Fu] established the abundance theorem for pairs $(X, \Delta)$ where $X$ is a smooth projective surface and $\Delta$ is a $\mathbb{Q}$-boundary, that is, where $\Delta$ is a $\mathbb{Q}$-divisor such that, for the prime decomposition $\Delta=\sum_{j \in J} \delta_{j} \Delta_{j}$, all the coefficients $\delta_{j}$ satisfy $0 \leq \delta_{j} \leq 1$. In characteristic 0 , Fujino [F2] generalized this result. More precisely, [F2] shows that the abundance theorem holds for pairs $(X, \Delta)$ where $X$ is a projective normal $\mathbb{Q}$-factorial surface and $\Delta$ is an $\mathbb{R}$-boundary. In this paper, we generalize this result to positive characteristic.

1.1.2. Minimal model theory. In characteristic 0, the minimal model theory has been developed by Kawamata, Kollár, Mori, Shokurov, and many others (see, e.g., [KoM], [KMM]). To establish fundamental theorems in minimal model theory, we use the Kodaira vanishing theorem and its generalizations.

However, in positive characteristic, counterexamples to the Kodaira vanishing theorem exist even in the case of dimension 2 (see $[R]$ ). In $[T]$, the author established a weak Kodaira vanishing theorem for positive characteristic surfaces and established a base-point-free theorem for klt surfaces. Kollár [Kol] established the contraction theorem for smooth 3-folds in positive characteristic. Kawamata [K] established the minimal model program for semistable 3 -folds in positive characteristic.

Fujino [F2] established the minimal model theory for $\mathbb{Q}$-factorial surfaces and $\log$ canonical surfaces in characteristic 0 . In the current article, we generalize this result to positive characteristic.

1.1.3. Keel's result. Keel's result (Theorem 2.2) is a key theorem in this paper. Thus, we summarize here some literature related to Keel's result. Theorem 2.2 is the surface version of [Ke1, Theorem 0.2]. Keel's proof depends on the Frobenius maps and the theory of the algebraic spaces. Note that Keel's result (Theorem 2.2) holds only in positive characteristic (see [Ke1, Section 3]). See [CMM] and [FT] for alternative proofs of [Ke1, Theorem 0.2] that do not depend on the theory of algebraic spaces. In [FT], Fujino and the author consider only the case of surfaces. 
The article [Ke1] also shows the base-point-free theorem for $\mathbb{Q}$-factorial 3 -folds over $\overline{\mathbb{F}}_{p}$ with nonnegative Kodaira dimension. Over $\overline{\mathbb{F}}_{p}$, we can often obtain some strong results. The reason is owing to Corollary 2.4 (see also [A], [Ke2], [M], [To]).

\subsection{Overview of contents}

Section 2 summarizes the notation and two known results: Keel's result (Theorem 2.2) and Fact 2.3, which play crucial roles in this paper.

In Section 3, we prove the case (QF) of Theorems 1.1 and 1.2. To show the case (QF) of Theorem 1.1, we establish the cone theorem and the contraction theorem. The cone theorem follows from Mori's bend-and-break lemma and the minimal resolution. We consider the bend-and-break method for proper normal surfaces in Section 3.1. The contraction theorem (Theorem 3.21) is obtained by Keel's result (Theorem 2.2).

To show the case (QF) of Theorem 1.2, we divide the argument into two cases: $k \neq \overline{\mathbb{F}}_{p}$ and $k=\overline{\mathbb{F}}_{p}$. We treat the case $k=\overline{\mathbb{F}}_{p}$ in Section 4 , so we prove Theorem 1.2 only for the case $k \neq \overline{\mathbb{F}}_{p}$. By a standard argument, we may assume that $\Delta$ is a $\mathbb{Q}$-divisor (Section 3.7). First, we prove that $\kappa:=$ $\kappa\left(X, K_{X}+\Delta\right) \geq 0$ (Theorem 3.30). This follows from the same argument as in [F2, Theorem 5.1]. Second, we consider the three cases $\kappa=0, \kappa=1$, and $\kappa=2$. If $\kappa=1$, then the assertion follows from a more general known result (Proposition 3.23). By using Keel's result and the contraction theorem, we can prove the case of $\kappa=2$ (Proposition 3.29). In the case where $\kappa=0$ (Theorem 3.34), we use the arguments in [F2] and [Fu], which depend on the classification of smooth surfaces.

In Section 4, we prove the case (FP) of Theorems 1.1 and 1.2. The case (FP) of Theorem 1.1 follows from Keel's result (Theorem 2.2) and Corollary 2.4. Proof of the case (FP) of Theorem 1.2 is almost the same as in [M, Theorem 2]. In Section 4, we also show that normal surfaces over $\overline{\mathbb{F}}_{p}$ are Q-factorial (Section 4.2).

In Section 5, we consider the case (LC) of Theorems 1.1 and 1.2. To show the case (LC) of Theorem 1.1, we describe the log canonical surface singularities by using results obtained in Section 3. This is discussed in Section 5.1. The case (LC) of Theorem 1.2 follows from a known result (see [Fu, (1.4) Main Theorem]).

In Section 6, we generalize the results in Sections 3-5 to relative situations. The relative version of Theorems 1.1 and 1.2 are Theorem 6.5 and Corollary 6.10, respectively. 
In the Appendices, we prove Theorems 1.3 and 1.4. Note that, in characteristic 0 , the base-point-free theorem holds for log canonical varieties (see [F1, Theorem 13.1]). Its proof heavily depends on the Kodaira vanishing theorem and its generalizations. Although, in positive characteristic, counterexamples to the Kodaira vanishing theorem exist (see $[R]$ ), we can establish the base-point-free theorem for surfaces (Theorem 1.3). Our proof depends on Keel's result (Theorem 2.2), the classification of smooth surfaces, and the Riemann-Roch theorem.

Theorem 1.4 follows from the relative Kawamata-Viehweg vanishing theorem for birational morphisms of surfaces.

\section{§2. Notation and known results}

\subsection{Notation}

2.1.1. Notation. We will freely use the notation and terminology in [KoM]. We will not distinguish the notation for line bundles, invertible sheaves, and Cartier divisors. For example, we will write $L+M$ for line bundles $L$ and $M$.

Throughout this article, we work over an algebraically closed field $k$, whose characteristic char $k=: p$ is positive unless otherwise mentioned.

In this paper, a variety means an integral scheme which is separated and of finite type over $k$. A curve or a surface means a variety whose dimension is 1 or 2 , respectively.

Let $D$ be an $\mathbb{R}$-divisor, and let $D=\sum_{j \in J} d_{j} D_{j}$ be its prime decomposition. For a real number $a$, we define $D \geq a$ by $d_{j} \geq a$ for all $j \in J$. We define $D \leq a, D>a$, and $D<a$ in the same way.

We say that $D$ is an $\mathbb{R}$-boundary (resp., a $\mathbb{Q}$-boundary) if $D$ is an $\mathbb{R}$-divisor (resp., a $\mathbb{Q}$-divisor) and if $0 \leq D \leq 1$.

Definition 2.1 (Semiample $\mathbb{R}$-divisors). Let $\pi: X \rightarrow S$ be a proper morphism between varieties. Let $D$ be an $\mathbb{R}$-Cartier $\mathbb{R}$-divisor. We say that $D$ is $\pi$-semiample if

$$
D=\sum_{1 \leq i \leq N} d_{i} D_{i},
$$

where $d_{i} \in \mathbb{R}_{\geq 0}$ and $D_{i}$ is a $\pi$-semiample Cartier divisor for every $i$ (for more details, see [F1, Section 4]).

\subsection{Known results}

2.2.1. Keel's result. In positive characteristic, we cannot use the Kodaira vanishing theorem, but we can use the following theorem by Keel. The 
following assertion is the surface version of the original theorem by Keel. (For an alternative proof, see [CMM] and [FT, Section 2].)

TheOREM 2.2 (Keel's result). Let $X$ be a projective normal surface over an algebraically closed field $k$ of positive characteristic. Let $L$ be a nef and big line bundle. Let $E(L)$ be the reduced subscheme whose support is the union of all the curves $C$ with $L \cdot C=0$. Then, $L$ is semiample if and only if $\left.L\right|_{E(L)}$ is semiample.

Proof. For a proof, see [Ke1, Theorem 0.2].

2.2.2. Difference between $k \neq \overline{\mathbb{F}}_{p}$ and $k=\overline{\mathbb{F}}_{p}$. In this paper, we often divide the argument into the two cases $k \neq \overline{\mathbb{F}}_{p}$ and $k=\overline{\mathbb{F}}_{p}$. The reason for this comes from the following fact.

FACT 2.3. Let $k$ be an algebraically closed field of arbitrary characteristic.

(1) If $k \neq \overline{\mathbb{F}}_{p}$, all abelian varieties over $k$ have infinite rank.

(2) If $k=\overline{\mathbb{F}}_{p}$, all group schemes of finite type over $\overline{\mathbb{F}}_{p}$ are torsion groups.

Proof. (1) For a proof, see [FJ, Theorem 10.1].

(2) Let $X$ be a group scheme of finite type over $\overline{\mathbb{F}}_{p}$. Let $P$ be a closed point of $X$. Then we see that $P$ and $X$ are defined over a finite field. Thus, we can consider $P$ as a rational point of a group scheme of finite type over a finite field. Since this group is finite, $P$ is a torsion.

As a corollary, we obtain the following information on the line bundles of varieties over $\overline{\mathbb{F}}_{p}$.

Corollary 2.4. Let $X$ be a projective variety over $\overline{\mathbb{F}}_{p}$, and let $D$ be a Cartier divisor. If $D \equiv 0$, then $D$ is a torsion in $\operatorname{Pic} X$.

Proof. Consider the Picard space of $X$, and apply Fact 2.3 (for more details, see [Ke1, Lemma 2.16]).

\section{§3. Q-factorial surfaces}

\subsection{Bend and break}

In this section, we consider Mori's bend-and-break method for proper normal surfaces. We use the following intersection theory for normal surfaces by Mumford [Mu1]. 
Definition 3.1 (Intersection theory by Mumford [Mu1]). Let $X$ be a normal surface, and let $f: X^{\prime} \rightarrow X$ be a resolution of singularities. Let $E_{1}, \ldots, E_{n}$ be the exceptional curves of $f$. Let $C$ be a proper curve in $X$, and let $D$ be an $\mathbb{R}$-divisor on $X$. Let $C^{\prime}$ and $D^{\prime}$ be their proper transforms, respectively.

(1) We define $f^{*} D:=D^{\prime}+\sum e_{i} E_{i}$, where all $e_{i}$ are real numbers uniquely determined by the linear equations $\left(D^{\prime}+\sum e_{i} E_{i}\right) \cdot E_{j}=0$ for $j=1, \ldots, n$. Note that the intersection matrix $\left(E_{i} \cdot E_{j}\right)$ is negative definite (see $[\mathrm{KoM}$, Lemma 3.40]).

(2) We define the intersection pairing by $C \cdot D:=f^{*} C \cdot f^{*} D=C^{\prime} \cdot f^{*} D$.

(3) If $X$ is proper, then we can naturally extend this intersection theory to Weil divisors with $\mathbb{Q}$ or $\mathbb{R}$ coefficients by linearity.

Definition 3.2. Let $X$ be a proper normal surface, and let $D$ and $D^{\prime}$ be $\mathbb{R}$-divisors. We denote $D \equiv_{\text {Mum }} D^{\prime}$ if $D \cdot C=D^{\prime} \cdot C$ for every curve $C$ in $X$.

Let us define the bend and break. This is the key method for the proof of the cone theorem.

Definition 3.3 (Bend and break). Let $X$ be a proper normal surface. We say that $X$ satisfies bend and break if $X$ satisfies the following two conditions.

(BB1) If $Z$ is a rational curve in $X$, then $Z \equiv_{\text {Mum }} Z_{1}+\cdots+Z_{r}$, where each $Z_{i}$ is a rational curve and $-Z_{i} \cdot K_{X} \leq 3$.

(BB2) Let $C$ be a curve in $X$ with $C \cdot K_{X}<0$. Then for an arbitrary point $c_{0} \in C \backslash \operatorname{Sing} X$, there exists a positive integer $n\left(X, C, c_{0}\right)$ with the following conditions. For an arbitrary positive integer $n$ with $n \geq n\left(X, C, c_{0}\right)$, there exist a nonnegative integer $\alpha_{n}$, a curve $C_{n}$, and an effective 1-cycle $Z_{n}$ with the following four conditions:

(a) $p^{n} C \equiv_{\text {Mum }} \alpha_{n} C_{n}+Z_{n}$;

(b) $Z_{n}=Z_{n, 1}+\cdots+Z_{n, r_{n}}$, where each $Z_{n, i}$ is a rational curve;

(c) $-\alpha_{n} C_{n} \cdot K_{X} \leq 2 g\left(C_{\text {normal }}\right)$, where $C_{\text {normal }}$ is the normalization of $C$;

(d) $c_{0} \in \operatorname{Supp} Z_{n}$.

The smooth case is the original bend and break proved by Mori, as follows.

Proposition 3.4. If $X$ is a projective smooth surface, then $X$ satisfies bend and break.

Proof. For a proof, see [Mo1, Theorems 4 and 5] and their proofs. 
Using this result, we extend the bend and break to the proper normal surfaces.

Proposition 3.5. If $X$ is a proper normal surface, then $X$ satisfies bend and break.

Proof. Let $f: X^{\prime} \rightarrow X$ be the minimal resolution, and let $K_{X^{\prime}}+\sum e_{i} E_{i}=$ $f^{*}\left(K_{X}\right)$, where all $E_{i}$ are exceptional curves and $e_{i} \geq 0$.

(BB1): Let $Z$ be a rational curve in $X$, and let $Z^{\prime}$ be its proper transform. Here, $Z^{\prime}$ is rational. Since $X^{\prime}$ is smooth, $X^{\prime}$ satisfies (BB1). Therefore, $Z^{\prime} \equiv Z_{1}^{\prime}+\cdots+Z_{r}^{\prime}$, all $Z_{i}^{\prime}$ are rational curves, and $-Z_{i}^{\prime} \cdot K_{X^{\prime}} \leq 3$. Apply $f_{*}$ to this equation. We obtain that $Z \equiv_{\text {Mum }} Z_{1}+\cdots+Z_{r}$, where $f_{*} Z_{i}^{\prime}=Z_{i}$. Note that $Z_{i}$ may be 0 . But if all of the $Z_{i}$ are 0 , then we have $Z \equiv_{\text {Mum }} 0$. This is a contradiction. Moreover, the above relation between $K_{X}$ and $K_{X^{\prime}}$ shows that $-Z_{i} \cdot K_{X} \leq 3$.

(BB2): Let $C$ be a curve in $X$ with $C \cdot K_{X}<0$, and let $C^{\prime}$ be its proper transform. We see that $C^{\prime} \cdot K_{X^{\prime}}<0$ from the above relation between canonical divisors. Let $c_{0}$ be an arbitrary element of $C \backslash \operatorname{Sing} X$, and let $c_{0}^{\prime}$ be a point of $C^{\prime}$ such that $f\left(c_{0}^{\prime}\right)=c_{0}$. Since $X^{\prime}$ is smooth, $X^{\prime}$ satisfies (BB2). Thus, we obtain $n\left(X^{\prime}, C^{\prime}, c_{0}^{\prime}\right), \alpha_{n}^{\prime}, C_{n}^{\prime}$, and $Z_{n}^{\prime}$. Let $n\left(X, C, c_{0}\right):=$ $n\left(X^{\prime}, C^{\prime}, c_{0}^{\prime}\right), \alpha_{n} C_{n}:=f_{*}\left(\alpha_{n}^{\prime} C_{n}^{\prime}\right)$, and $Z_{n}:=f_{*}\left(Z_{n}^{\prime}\right)$. It is easy to see that these satisfy (BB2).

From now on, let us generalize this result for pairs $(X, \Delta)$.

Definition $3.6\left(\left(K_{X}+\Delta\right)\right.$-bend and break). Let $X$ be a proper normal surface, and let $\Delta$ be an effective $\mathbb{R}$-divisor. Let $\Delta=\sum b_{i} B_{i}$ be its prime decomposition. We say that $(X, \Delta)$ satisfies $\left(K_{X}+\Delta\right)$-bend and break if $X$ and $\Delta$ satisfy the following two conditions.

(BB1) There exists a positive integer $L(X, \Delta)$ which satisfies both of the following conditions.

(1) If $Z$ is a rational curve in $X$, then $Z \equiv_{\text {Mum }} Z_{1}+\cdots+Z_{r}$, where all $Z_{i}$ are rational curves and $-Z_{i} \cdot\left(K_{X}+\Delta\right) \leq L(X, \Delta)$.

(2) If $B_{i}^{2}<0$, then $-B_{i} \cdot\left(K_{X}+\Delta\right) \leq L(X, \Delta)$.

(BB2) Let $C$ be a curve in $X$ with $C \cdot\left(K_{X}+\Delta\right)<0$ and $C \neq B_{i}$ for all $i$ such that $B_{i}^{2}<0$. Then, for an arbitrary point $c_{0} \in C \backslash \operatorname{Sing} X$, there exists a positive integer $n\left(X, \Delta, C, c_{0}\right)$ with the following conditions. For an arbitrary integer $n$ with $n \geq n\left(X, \Delta, C, c_{0}\right)$, there exist a nonnegative integer $\alpha_{n}$, a curve $C_{n}$, and an effective 1-cycle $Z_{n}$ with the following four conditions: 
(a) $p^{n} C \equiv_{\text {Mum }} \alpha_{n} C_{n}+Z_{n}$;

(b) $Z_{n}=Z_{n, 1}+\cdots+Z_{n, r_{n}}$, where all $Z_{n, i}$ are rational curves;

(c) $-\alpha_{n} C_{n} \cdot\left(K_{X}+\Delta\right) \leq 2 g\left(C_{\text {normal }}\right)$, where $C_{\text {normal }}$ is the normalization of $C$ or $C_{n}=B_{i}$ for some $i$ such that $B_{i}^{2}<0$;

(d) $c_{0} \in \operatorname{Supp} Z_{n}$.

We obtain the following main result in this section.

THEOREM 3.7. If $X$ is a proper normal surface, and if $\Delta$ is an effective $\mathbb{R}$-divisor, then $(X, \Delta)$ satisfies $\left(K_{X}+\Delta\right)$-bend and break.

Proof. We write the prime decomposition $\Delta=\sum b_{i} B_{i}$.

(BB1): Let

$$
L(X, \Delta):=\max \left(\{3\} \cup\left\{-\left(K_{X}+\Delta\right) \cdot B_{\mu}\right\}\right),
$$

where $B_{\mu}$ ranges over the prime components of $\Delta$ with $B_{\mu}^{2}<0$. We check conditions (1) and (2). Condition (2) is obvious. Thus, let us prove (1). Let $Z$ be a rational curve in $X$. By Proposition 3.5, we have $Z \equiv_{\text {Mum }} Z_{1}+\cdots+Z_{r}$, where any $Z_{j}$ is rational and satisfies $-Z_{j} \cdot K_{X} \leq 3$. If $Z_{j}=B_{\mu}$ with $B_{\mu}^{2}<0$, then we obtain $-Z_{j} \cdot\left(K_{X}+\Delta\right) \leq L(X, \Delta)$. If $Z_{j} \neq B_{\mu}$, then we have

$$
-Z_{j} \cdot\left(K_{X}+\Delta\right) \leq-Z_{j} \cdot K_{X} \leq 3 \leq L(X, \Delta)
$$

(BB2): Let $C$ be a curve in $X$ with $C \cdot\left(K_{X}+\Delta\right)<0$ and with $C \neq B_{i}$ for all $B_{i}$ such that $B_{i}^{2}<0$. Then we obtain the following inequalities:

$$
C \cdot K_{X} \leq C \cdot\left(K_{X}+\Delta\right)<0
$$

By Proposition 3.5, we can use the bend and break in the sense of Definition 3.3. Let

$$
n\left(X, \Delta, C, c_{0}\right):=n\left(X, C, c_{0}\right) .
$$

This satisfies the four conditions of (BB2) of Definition 3.6. Indeed, conditions (a), (b), and (d) are obvious. We consider (c). If $C_{n} \neq B_{i}$ for all $B_{i}$ such that $B_{i}^{2}<0$, then we have

$$
-\alpha_{n} C_{n} \cdot\left(K_{X}+\Delta\right) \leq-\alpha_{n} C_{n} \cdot K_{X} \leq 2 g\left(C_{\text {normal }}\right) .
$$

This completes the proof.

Let us calculate $L(X, \Delta)$ in the case where $\Delta$ is an $\mathbb{R}$-boundary. 
Proposition 3.8. Let $X$ be a proper normal surface, and let $\Delta$ be an $\mathbb{R}$ boundary. Then $(X, \Delta)$ satisfies $\left(K_{X}+\Delta\right)$-bend and break for $L(X, \Delta)=3$.

Proof. By the proof of Theorem 3.7, $(X, \Delta)$ satisfies $\left(K_{X}+\Delta\right)$-bend and break for

$$
L(X, \Delta)=\max \left(\{3\} \cup\left\{-\left(K_{X}+\Delta\right) \cdot B_{\mu}\right\}\right),
$$

where $B_{\mu}$ ranges over the prime components of $\Delta$ with $B_{\mu}^{2}<0$. Thus, the assertion follows from the following lemma.

Lemma 3.9. Let $X$ be a normal surface, and let $\Delta$ be an $\mathbb{R}$-boundary. If $C$ is a proper curve in $X$ such that $C^{2} \leq 0$, then $-\left(K_{X}+\Delta\right) \cdot C \leq 2$.

Proof. Let $f: Y \rightarrow X$ be the minimal resolution, and let $C_{Y}$ be the proper transform of $C$. We define $\Delta_{Y}$ by

$$
K_{Y}+C_{Y}+\Delta_{Y}=f^{*}\left(K_{X}+C\right)
$$

Note that $\Delta_{Y} \geq 0$ and that $C_{Y} \not \subset \operatorname{Supp} \Delta_{Y}$. Then, we see that $\left(K_{Y}+C_{Y}\right)$. $C_{Y} \geq-2$. We obtain

$$
\begin{aligned}
\left(K_{X}+\Delta\right) \cdot C & \geq\left(K_{X}+C\right) \cdot C \\
& =f^{*}\left(K_{X}+C\right) \cdot C_{Y} \\
& =\left(K_{Y}+C_{Y}+\Delta_{Y}\right) \cdot C_{Y} \\
& \geq\left(K_{Y}+C_{Y}\right) \cdot C_{Y} \\
& \geq-2 .
\end{aligned}
$$

\subsection{Cone theorem}

In this section we prove the cone theorem. We use the bend-and-break method in the sense of Definition 3.6. Thus, in this section we use the notation in Definition 3.6.

Here, let us recall the definition of the Kleiman-Mori cone.

Definition 3.10. Let $X$ be a projective variety. Then we define

$$
\begin{aligned}
N(X) & :=\left\{r_{1} Z_{1}+\cdots+r_{s} Z_{s} \mid r_{i} \in \mathbb{R} \text { and } Z_{i} \text { is a curve in } X\right\} \equiv \\
N E(X) & :=\left\{\left[r_{1} Z_{1}+\cdots+r_{s} Z_{s}\right] \mid r_{i} \geq 0, Z_{i} \text { is a curve in } X\right\} \subset N(X),
\end{aligned}
$$

where $\left[r_{1} Z_{1}+\cdots+r_{s} Z_{s}\right]$ means the equivalence class. 
Note that $N(X)$ is the quotient space by $\equiv$. Although we often use the intersection theory by Mumford, we do not take the quotient by $\equiv_{\text {Mum. }}$. The numerical equivalence $\equiv$ is induced by the intersections only with $\mathbb{R}$-Cartier divisors.

In this section, we use the following lemma repeatedly.

Lemma 3.11. Let $a, b \in \mathbb{R}$ and $c, d \in \mathbb{R}_{>0}$. Then,

$$
\frac{a+b}{c+d} \leq \max \left\{\frac{a}{c}, \frac{b}{d}\right\} .
$$

Proof. The proof is easy. Thus, we omit it.

The following lemma is key to this section.

Lemma 3.12. Let $X$ be a projective normal surface, and let $\Delta$ be an effective $\mathbb{R}$-divisor such that $K_{X}+\Delta$ is $\mathbb{R}$-Cartier. Let $\Delta=\sum b_{i} B_{i}$ be the prime decomposition. Let $H$ be an $\mathbb{R}$-Cartier ample $\mathbb{R}$-divisor. If $C$ is a curve in $X$ such that $C \cdot\left(K_{X}+\Delta\right)<0$, then there exists a curve $E$ in $X$ with the following properties:

(1) $E$ is rational or $E=B_{j}$ for some $j$ such that $B_{j}^{2}<0$;

(2) $0<-E \cdot\left(K_{X}+\Delta\right) \leq L(X, \Delta)$;

$$
\frac{-C \cdot\left(K_{X}+\Delta\right)}{C \cdot H} \leq \frac{-E \cdot\left(K_{X}+\Delta\right)}{E \cdot H} .
$$

(The following proof is very similar to that of [KoM, Theorem 1.13].)

Proof of Lemma 3.12. In this proof, we use the notation (BB1) and (BB2) in the sense of Definition 3.6. First, if $C=B_{j}$ with $B_{j}^{2}<0$, then the assertion is obvious. We may assume that $C \neq B_{j}$ for all $B_{j}$ with $B_{j}^{2}<0$. Then, we can use (BB2). However, since we do not use $c_{0}$, we fix $c_{0} \in C \backslash \operatorname{Sing} X$. Set $C_{n}^{\prime}:=\alpha_{n} C_{n}$. We consider the following number:

$$
\begin{aligned}
M & :=\frac{-C \cdot\left(K_{X}+\Delta\right)}{C \cdot H} \\
& =\frac{-p^{n} C \cdot\left(K_{X}+\Delta\right)}{p^{n} C \cdot H} \\
& =\frac{-C_{n}^{\prime} \cdot\left(K_{X}+\Delta\right)-Z_{n} \cdot\left(K_{X}+\Delta\right)}{C_{n}^{\prime} \cdot H+Z_{n} \cdot H} \\
& =\frac{a_{n}+b_{n}}{c_{n}+d_{n}}
\end{aligned}
$$


where $a_{n}, b_{n}, c_{n}$, and $d_{n}$ are defined by

$$
\begin{aligned}
a_{n} & :=-C_{n}^{\prime} \cdot\left(K_{X}+\Delta\right), \\
b_{n} & :=-Z_{n} \cdot\left(K_{X}+\Delta\right), \\
c_{n} & :=C_{n}^{\prime} \cdot H, \\
d_{n} & :=Z_{n} \cdot H .
\end{aligned}
$$

STEP 1. In this step, we reduce the proof to the case where $\alpha_{n}>0$ for all $n \gg 0$.

Assume that there is a positive integer $n$ such that $n \geq n\left(X, \Delta, C, c_{0}\right)$ and $\alpha_{n}=0$. Then we have

$$
\frac{-C \cdot\left(K_{X}+\Delta\right)}{C \cdot H}=\frac{-Z_{n} \cdot\left(K_{X}+\Delta\right)}{Z_{n} \cdot H} \leq \frac{-Z_{n, i} \cdot\left(K_{X}+\Delta\right)}{Z_{n, i} \cdot H}
$$

for some $i$ by Lemma 3.11. Moreover, by (BB1) and Lemma 3.11, we obtain the desired result.

STEP 2. In this step, we reduce the proof to the case where

$$
a_{n}=-\alpha_{n} C_{n} \cdot\left(K_{X}+\Delta\right) \leq 2 g\left(C_{\text {normal }}\right)
$$

for all $n \gg 0$.

Suppose the contrary. Then, by condition (c) of (BB2), we obtain $C_{n}=B_{j}$ for some $j$ such that $B_{j}^{2}<0$. By Lemma 3.11, we have the following equality:

$$
\begin{aligned}
\frac{-C \cdot\left(K_{X}+\Delta\right)}{C \cdot H} & =\frac{-\alpha_{n} B_{j} \cdot\left(K_{X}+\Delta\right)-Z_{n} \cdot\left(K_{X}+\Delta\right)}{\alpha_{n} B_{j} \cdot H+Z_{n} \cdot H} \\
& \leq \max \left\{\frac{-B_{j} \cdot\left(K_{X}+\Delta\right)}{B_{j} \cdot H}, \frac{-Z_{n} \cdot\left(K_{X}+\Delta\right)}{Z_{n} \cdot H}\right\} .
\end{aligned}
$$

If

$$
\frac{-C \cdot\left(K_{X}+\Delta\right)}{C \cdot H} \leq \frac{-B_{j} \cdot\left(K_{X}+\Delta\right)}{B_{j} \cdot H},
$$

then this is the desired result. If

$$
\frac{-C \cdot\left(K_{X}+\Delta\right)}{C \cdot H} \leq \frac{-Z_{n} \cdot\left(K_{X}+\Delta\right)}{Z_{n} \cdot H},
$$

then, by (BB1) and Lemma 3.11, we obtain the desired result.

From now on, we consider the asymptotic behaviors of $a_{n}, b_{n}, c_{n}$, and $d_{n}$. 
STEP 3. The sequence $a_{n}$ is bounded, and the sequence $b_{n}$ is not bounded. Indeed, the boundedness of $a_{n}$ follows from Step 2. Since $a_{n}+b_{n}=-p^{n} C$. $\left(K_{X}+\Delta\right)$ is not bounded, $b_{n}$ is not bounded.

STEP 4. In this step, we prove that for an arbitrary positive real number $\epsilon$, there exists a curve $E$ in $X$ with the following properties:

$(1)^{\prime} E$ is rational;

$(2)^{\prime} \quad 0<-E \cdot\left(K_{X}+\Delta\right) \leq L(X, \Delta)$;

$(3)^{\prime}$

$$
M-\epsilon<\frac{-E \cdot\left(K_{X}+\Delta\right)}{E \cdot H} .
$$

If $a_{n} / c_{n}<M$ for some $n \gg 0$, then we have $b_{n} / d_{n} \geq M$, which gives us the desired result by (BB1) and Lemma 3.11. Thus, we may assume that $a_{n} / c_{n} \geq M$ for all $n \gg 0$. Then, since $a_{n}$ is bounded, so is $c_{n}$ because $M$ is a positive number. Because $c_{n}+d_{n}=p^{n} C \cdot H, d_{n}$ is not bounded. Therefore, for sufficiently large $n$, we obtain

$$
\frac{b_{n}}{d_{n}}+\epsilon>\frac{a_{n}+b_{n}}{d_{n}}>\frac{a_{n}+b_{n}}{c_{n}+d_{n}}=M
$$

By (BB1) and Lemma 3.11, there exists a rational curve $E$ with the desired properties.

STEP 5. We take an arbitrary positive real number $\epsilon$ with $0<\epsilon \leq M / 2$. Then, by Step 4, we obtain

$$
E \cdot H<\frac{-E \cdot\left(K_{X}+\Delta\right)}{M-\epsilon} \leq \frac{L(X, \Delta)}{M / 2}=\frac{2 L(X, \Delta)}{M} .
$$

Since $H$ is ample, the subset in numerical classes of effective 1-cycles in $X$ with integral coefficients

$$
\left\{[E] \mid E \cdot H<\frac{2 L(X, \Delta)}{M}\right\}
$$

has only finitely many members. Therefore, the set

$$
\left\{\frac{-E \cdot\left(K_{X}+\Delta\right)}{E \cdot H} \mid E \cdot H<\frac{2 L(X, \Delta)}{M} \text { and } E \text { satisfies }(1)^{\prime},(2)^{\prime}\right\}
$$

is also a finite set, because $K_{X}+\Delta$ is $\mathbb{R}$-Cartier. Take a sufficiently small $\epsilon>0$. Then, by Step 4 , we obtain a rational curve $E$ in $X$ such that

$$
E \text { satisfies }(1)^{\prime},(2)^{\prime} \text { and } \frac{-E \cdot\left(K_{X}+\Delta\right)}{E \cdot H} \geq M .
$$

This completes the proof. 
Let us prove the cone theorem.

Theorem 3.13 (Cone theorem). Let $X$ be a projective normal surface, and let $\Delta$ be an effective $\mathbb{R}$-divisor such that $K_{X}+\Delta$ is $\mathbb{R}$-Cartier. Let $\Delta=\sum b_{i} B_{i}$ be the prime decomposition. Let $H$ be an $\mathbb{R}$-Cartier ample $\mathbb{R}$ divisor. Then the following assertions hold:

(1) $\overline{N E}(X)=\overline{N E}(X)_{K_{X}+\Delta \geq 0}+\sum \mathbb{R}_{\geq 0}\left[C_{i}\right]$;

(2) $\overline{N E}(X)=\overline{N E}(X)_{K_{X}+\Delta+H \geq 0}+\sum_{\text {finite }} \mathbb{R}_{\geq 0}\left[C_{i}\right]$;

(3) each $C_{i}$ in (1) and (2) is rational or $C_{i}=B_{j}$ for some $B_{j}$ with $B_{j}^{2}<0$;

(4) each $C_{i}$ in (1) and (2) satisfies $0<-C_{i} \cdot\left(K_{X}+\Delta\right) \leq L(X, \Delta)$.

This proof is essentially the same as that of [KoM, Theorem 1.24].

Proof of Theorem 3.13. (1) Let $W$ be the right-hand side in (1); that is,

$$
W:=\overline{N E}(X)_{\left(K_{X}+\Delta\right) \geq 0}+\sum_{C_{i} \text { satisfies }(3),(4)} \mathbb{R}_{\geq 0}\left[C_{i}\right] .
$$

Note that $W$ is a closed set by the same proof as in [Ko2, Chapter 3, Theorem 1.2]. We would like to prove that $\overline{N E}(X)=W$. The inclusion $\overline{N E}(X) \supset W$ is clear. Let us assume that $\overline{N E}(X) \supsetneq W$ and derive a contradiction. Then we can find a Cartier divisor $D$ which is positive on $W \backslash 0$ and which is negative on some element of $\overline{N E}(X)$. Let $\mu$ be a positive real number such that $H+\mu D$ is nef and $H+\mu^{\prime} D$ is ample for all positive real numbers $\mu^{\prime}$ with $\mu^{\prime}<\mu$. Then we can take a 1-cycle $Z$ with $Z \in \overline{N E}(X) \backslash\{0\}$ and $(H+\mu D) \cdot Z=0$. Since $Z \cdot H>0$ means that $Z \cdot D<0, Z$ is not in $W$. By the definition of $W$, we obtain $Z \cdot\left(K_{X}+\Delta\right)<0$. Because $Z$ is an element of $\overline{N E}(X)$, there exist effective 1-cycles $Z_{k}=\sum a_{k, j} Z_{k, j}$ such that the limit of $Z_{k}$ is $Z$. Take an arbitrary positive real number $\mu^{\prime}$ with $\mu^{\prime}<\mu$. By the ampleness of $H+\mu^{\prime} D$, we have

$$
\max _{j} \frac{-Z_{k, j} \cdot\left(K_{X}+\Delta\right)}{Z_{k, j} \cdot\left(H+\mu^{\prime} D\right)} \geq \frac{-Z_{k} \cdot\left(K_{X}+\Delta\right)}{Z_{k} \cdot\left(H+\mu^{\prime} D\right)} .
$$

We may assume that the maximum on the left-hand side occurs when $j$ is 0 . By Lemma 3.12, we obtain

$$
\frac{-E_{k} \cdot\left(K_{X}+\Delta\right)}{E_{k} \cdot\left(H+\mu^{\prime} D\right)} \geq \frac{-Z_{k, 0} \cdot\left(K_{X}+\Delta\right)}{Z_{k, 0} \cdot\left(H+\mu^{\prime} D\right)} \geq \frac{-Z_{k} \cdot\left(K_{X}+\Delta\right)}{Z_{k} \cdot\left(H+\mu^{\prime} D\right)} .
$$


Here, $E_{k}$ satisfies (3) and (4). Thus, we have $E_{k} \in W$, and this means that $E_{k} \cdot D \geq 0$. Therefore, we have

$$
\frac{-E_{k} \cdot\left(K_{X}+\Delta\right)}{E_{k} \cdot H} \geq \frac{-E_{k} \cdot\left(K_{X}+\Delta\right)}{E_{k} \cdot\left(H+\mu^{\prime} D\right)} \geq \frac{-Z_{k} \cdot\left(K_{X}+\Delta\right)}{Z_{k} \cdot\left(H+\mu^{\prime} D\right)} .
$$

Take a large positive number $r$ such that $r H+\left(K_{X}+\Delta\right)$ is ample. This shows that

$$
r>\frac{-E_{k} \cdot\left(K_{X}+\Delta\right)}{E_{k} \cdot H}
$$

Combining the inequalities, we obtain

$$
r>\frac{-Z_{k} \cdot\left(K_{X}+\Delta\right)}{Z_{k} \cdot\left(H+\mu^{\prime} D\right)}
$$

Recall that we choose $\mu^{\prime}$ as an arbitrary positive real number with $\mu^{\prime}<\mu$. By taking the limit $\mu^{\prime}$ to $\mu$, we obtain

$$
r \geq \frac{-Z_{k} \cdot\left(K_{X}+\Delta\right)}{Z_{k} \cdot(H+\mu D)}
$$

Moreover, by taking the limit $k$ to $\infty$, we obtain

$$
r \geq \lim _{k \rightarrow \infty} \frac{-Z_{k} \cdot\left(K_{X}+\Delta\right)}{\left(Z_{k} \cdot H+\mu D\right)}=\frac{(\text { positive })}{+0}=+\infty .
$$

This is a contradiction. This completes the proof of (1).

(2) If $C_{i} \cdot\left(K_{X}+\Delta+H\right)<0$, then we have

$$
C_{i} \cdot H<-C_{i} \cdot\left(K_{X}+\Delta\right) \leq L(X, \Delta)
$$

There are only finitely many numerical classes of curves like this. This shows (2). The remaining assertions (3) and (4) have already been proved in the above arguments.

REMARK 3.14. In Theorem 3.13, $L(X, \Delta)$ gives an upper bound of length of extremal rays. By the proof of Theorem 3.7,

$$
L(X, \Delta):=\max \left(\{3\} \cup\left\{-\left(K_{X}+\Delta\right) \cdot B_{\mu}\right\}\right),
$$

where $B_{\mu}$ ranges over the prime components of $\Delta$ with $B_{\mu}^{2}<0$. In the case where $\Delta$ is an $\mathbb{R}$-boundary, we can set $L(X, \Delta)=3$ by Proposition 3.8. 
Moreover, in the case where $\Delta$ is an $\mathbb{R}$-boundary, every $\left(K_{X}+\Delta\right)$-negative extremal ray is generated by a rational curve.

Proposition 3.15. Let $X$ be a projective normal surface, and let $\Delta$ be an $\mathbb{R}$-boundary such that $K_{X}+\Delta$ is $\mathbb{R}$-Cartier. If $R$ is a $\left(K_{X}+\Delta\right)$-negative extremal ray of $\overline{N E}(X)$, then $R=\mathbb{R}_{\geq 0}[C]$, where $C$ is a rational curve such that $-\left(K_{X}+\Delta\right) \cdot C \leq 3$.

Proof. By Theorem 3.13 and Remark 3.14, we can write $R=\mathbb{R}_{\geq 0}[C]$, where $C$ is a curve such that $-\left(K_{X}+\Delta\right) \cdot C \leq 3$ and such that $C$ is rational or $C^{2}<0$. Assume that $C^{2}<0$. Then, we obtain

$$
\left(K_{X}+C\right) \cdot C \leq\left(K_{X}+\Delta\right) \cdot C<0 .
$$

Thus, the assertion follows from the following lemma.

Lemma 3.16. Let $X$ be a normal surface, and let $C$ be a proper curve in $X$. If $\left(K_{X}+C\right) \cdot C<0$, then $C$ is a rational curve.

Proof. Let $f: Y \rightarrow X$ be the minimal resolution, and let $C_{Y}$ be the proper transform of $C$. We define $\Delta_{Y}$ by $K_{Y}+C_{Y}+\Delta_{Y}=f^{*}\left(K_{X}+C\right)$. Then, we see that

$$
\left(K_{Y}+C_{Y}\right) \cdot C_{Y} \leq\left(K_{Y}+C_{Y}+\Delta_{Y}\right) \cdot C_{Y}=\left(K_{X}+C\right) \cdot C<0 .
$$

Then, since $C_{Y}$ is a rational curve, so is $C$.

\subsection{Results on adjunction formulas}

In this section, we summarize results on adjunction formulas.

Proposition 3.17. Let $X$ be a projective normal surface, and let $C$ be a curve in $X$. Then, there exists an exact sequence

$$
\left.0 \rightarrow \mathcal{T} \rightarrow \omega_{X}(C)\right|_{C} \rightarrow \omega_{C} \rightarrow 0
$$

where $\mathcal{T}$ is the torsion subsheaf of $\left.\omega_{X}(C)\right|_{C}$.

Proof. For a proof, see [F2, Lemma 4.4].

Using this adjunction formula, we obtain the following result on global sections.

Lemma 3.18. Let $X$ be a projective normal surface, and let $C$ be a curve in $X$. Fix a positive integer $r \in \mathbb{Z}_{>0}$. If $H^{1}\left(C, \mathcal{O}_{C}\right) \neq 0$, then $H^{0}(C$, $\left.\left.\omega_{X}(C)^{[r]}\right|_{C}\right) \neq 0$, where $\omega_{X}(C)^{[r]}$ is the double dual of $\omega_{X}(C)^{\otimes r}$. 
Proof. We consider the exact sequence

$$
\left.0 \rightarrow \mathcal{T} \rightarrow \omega_{X}(C)\right|_{C} \rightarrow \omega_{C} \rightarrow 0
$$

Since $\mathcal{T}$ is a skyscraper sheaf, we have $H^{1}(C, \mathcal{T})=0$. By $H^{0}\left(C, \omega_{C}\right) \neq 0$, we obtain $H^{0}\left(C,\left.\omega_{X}(C)\right|_{C} / \mathcal{T}\right) \neq 0$. Thus, there exists a map

$$
\left.\mathcal{O}_{C} \rightarrow \omega_{X}(C)\right|_{C}
$$

such that this is injective on some nonempty open set. Therefore, we obtain a map

$$
\left.\mathcal{O}_{C} \rightarrow \omega_{X}(C)^{\otimes r}\right|_{C}
$$

which is injective on some nonempty open set. On the other hand, there is a natural map

$$
\left.\left.\omega_{X}(C)^{\otimes r}\right|_{C} \rightarrow \omega_{X}(C)^{[r]}\right|_{C}
$$

which is bijective on some nonempty open set. Combining these maps, we have the map

$$
\left.\mathcal{O}_{C} \rightarrow \omega_{X}(C)^{[r]}\right|_{C}
$$

which is injective on some nonempty open set. Thus, the kernel $K$ of this map is a torsion subsheaf of $\mathcal{O}_{C}$. Then, we have $K=0$. Therefore, we obtain an injection $\left.\mathcal{O}_{C} \hookrightarrow \omega_{X}(C)^{[r]}\right|_{C}$. This means that $H^{0}\left(C,\left.\omega_{X}(C)^{[r]}\right|_{C}\right) \neq 0$. $\square$

Using this lemma, we obtain the following theorem, which plays a crucial role in this paper.

Theorem 3.19. Let $X$ be a projective normal surface, and let $C$ be a curve in $X$ such that $r\left(K_{X}+C\right)$ is Cartier for some positive integer $r$.

(1) If $C \cdot\left(K_{X}+C\right)<0$, then $C \simeq \mathbb{P}^{1}$.

(2) If $C \cdot\left(K_{X}+C\right)=0$, then $C \simeq \mathbb{P}^{1}$ or $\mathcal{O}_{C}\left(\left(K_{X}+C\right)^{[r]}\right) \simeq \mathcal{O}_{C}$.

Proof. (1) Since $C \cdot\left(K_{X}+C\right)<0$ means that $H^{0}\left(C,\left.\omega_{X}(C)^{[r]}\right|_{C}\right)=0$, the curve $C$ must be $\mathbb{P}^{1}$ by Lemma 3.18 .

(2) Assume that $C \not \mathbb{P}^{1}$. Then we can apply Lemma 3.18 and obtain $H^{0}\left(C,\left.\omega_{X}(C)^{[r]}\right|_{C}\right) \neq 0$. By $C \cdot\left(K_{X}+C\right)=0$, we have $\left.\omega_{X}(C)^{[r]}\right|_{C} \simeq \mathcal{O}_{C}$. 


\subsection{Contraction theorem}

In this section, we show that extremal rays are contractible for $\mathbb{Q}$-factorial surfaces with $\mathbb{R}$-boundaries. First, we consider the following theorem, which we will use later.

THEOREM 3.20. Let $k$ be an algebraically closed field of arbitrary characteristic. Let $\pi: Y \rightarrow B$ be a surjective morphism over $k$ from a smooth projective surface $Y$ to a smooth projective irrational curve $B$. Let $f: Y \rightarrow X$ be a birational morphism to a projective normal $\mathbb{Q}$-factorial surface. If $k$ is not the algebraic closure of a finite field, then all $f$-exceptional curves are $\pi$-vertical.

This proof is essentially due to [F2, Lemma 5.2].

Proof of Theorem 3.20. We assume that $C$ is an $f$-exceptional curve with $\pi(C)=B$ and want to derive a contradiction. We may assume that $C$ is smooth by taking a sequence of blowups of singular points of $C$. We have

$$
\begin{aligned}
& \left.\pi\right|_{C}: C \longrightarrow B, \\
& \operatorname{Pic}^{0} C \stackrel{\left(\left.\pi\right|_{C}\right)^{*}}{\longleftarrow} \operatorname{Pic}^{0} B .
\end{aligned}
$$

We prove that the image $\left(\left.\pi\right|_{C}\right)^{*}\left(\mathrm{Pic}^{0} B\right)$ is an abelian group whose rank is infinite. By considering $\left(\left.\pi\right|_{C}\right)^{*}$ as a morphism between Jacobian varieties, we see that $\left(\left.\pi\right|_{C}\right)^{*}\left(\mathrm{Pic}^{0} B\right)$ is an abelian variety. Note that the dimension of $\left(\left.\pi\right|_{C}\right)^{*}\left(\operatorname{Pic}^{0} B\right)$ as a scheme is not 0 by $\left(\left.\pi\right|_{C}\right)_{*} \circ\left(\left.\pi\right|_{C}\right)^{*}=\operatorname{deg}\left(\left.\pi\right|_{C}\right)$ and by the irrationality of $B$. Thus, by Fact 2.3 , the rank of $\left(\left.\pi\right|_{C}\right)^{*}\left(\operatorname{Pic}^{0} B\right)$ is infinite. Then, we have

$$
\left(\left.\pi\right|_{C}\right)^{*}\left(\mathrm{Pic}^{0} B\right) \otimes_{\mathbb{Z}} \mathbb{Q} \backslash \sum_{i=1}^{r} \mathbb{Q}\left(\left.E_{i}\right|_{C}\right) \neq \emptyset,
$$

where $E_{1}, \ldots, E_{r}$ are the $f$-exceptional curves. Therefore, we can take a $\mathbb{Q}$-divisor $D$ on $B$ such that

$$
\left.\left(\pi^{*} D\right)\right|_{C} \notin \sum_{i=1}^{r} \mathbb{Q}\left(\left.E_{i}\right|_{C}\right) .
$$

On the other hand, since $X$ is $\mathbb{Q}$-factorial, we obtain

$$
\pi^{*} D-f^{*} f_{*} \pi^{*} D \in \sum_{i=1}^{r} \mathbb{Q} E_{i} .
$$


Restricting this relation to $C$, we have the contradiction

$$
\left.\left(\pi^{*} D\right)\right|_{C} \in \sum_{i=1}^{r} \mathbb{Q}\left(\left.E_{i}\right|_{C}\right)
$$

because $C$ is $f$-exceptional.

Originally, [F2] uses this theorem to prove the nonvanishing theorem. We use this theorem not only for the nonvanishing theorem but also for the following contraction theorem.

Theorem 3.21 (Contraction theorem). Let $X$ be a projective normal $\mathbb{Q}$-factorial surface, and let $\Delta$ be an $\mathbb{R}$-boundary. Let $R=\mathbb{R}_{\geq 0}[C]$ be a $\left(K_{X}+\right.$ $\Delta)$-negative extremal ray. Then there exists a surjective morphism $\phi_{R}: X \rightarrow$ $Y$ to a projective variety $Y$ with the following properties.

(1) Let $C^{\prime}$ be a curve on $X$. Then $\phi_{R}\left(C^{\prime}\right)$ is one point if and only if $\left[C^{\prime}\right] \in R$.

(2) We have $\left(\phi_{R}\right)_{*}\left(\mathcal{O}_{X}\right)=\mathcal{O}_{Y}$.

(3) If $L$ is an invertible sheaf with $L \cdot C=0$, then $n L=\left(\phi_{R}\right)^{*} L_{Y}$ for some invertible sheaf $L_{Y}$ on $Y$ and for some positive integer $n$.

(4) We have $\rho(Y)=\rho(X)-1$.

(5) If $\operatorname{dim} Y=2$, then $Y$ is $\mathbb{Q}$-factorial.

We divide the proof into the three cases: $C^{2}>0, C^{2}=0$, and $C^{2}<0$.

Proof of the case where $C^{2}>0$. The inequality $C^{2}>0$ shows that $C$ is a nef and big divisor. Therefore, for an arbitrary curve $C^{\prime}$, there exists an effective Cartier divisor $E$ and positive integers $n$ and $m$ such that $n C \sim$ $m C^{\prime}+E$ by Kodaira's lemma. Since $C$ generates an extremal ray, we have $C^{\prime} \equiv q C$ for some rational number $q$. Recall that we choose $C^{\prime}$ as an arbitrary curve. Thus, we obtain $\rho(X)=1$ and $-K_{X}$ is ample. Then let $Y$ be one point, and properties (1), (2), and (4) are satisfied. We want to prove (3); that is, we must show that for a $\mathbb{Q}$-divisor $D$, if $D \equiv 0$, then $D$ is a torsion. It is sufficient to prove that $\kappa(X, D) \geq 0$. Thus, we assume that $\kappa(X, D)=-\infty$ and derive a contradiction. Let $f: X^{\prime} \rightarrow X$ be the minimal resolution, let $D^{\prime}=f^{*} D$, and let $K_{X^{\prime}}+E^{\prime}=f^{*} K_{X}$, where $E^{\prime}$ is an effective $f$-exceptional $\mathbb{Q}$-divisor. Then we obtain

$$
\begin{aligned}
\kappa\left(X^{\prime}, K_{X^{\prime}}\right) & \leq \kappa\left(X^{\prime}, K_{X^{\prime}}+E^{\prime}\right) \\
& =\kappa\left(X, K_{X}\right)=-\infty .
\end{aligned}
$$


First we prove that $X^{\prime}$ is an irrational ruled surface. By Serre duality, we obtain $h^{2}\left(X^{\prime}, D^{\prime}\right)=h^{0}\left(X^{\prime}, K_{X^{\prime}}-D^{\prime}\right)$. Moreover, we get

$$
\begin{aligned}
\kappa\left(X^{\prime}, K_{X^{\prime}}-D^{\prime}\right) & \leq \kappa\left(X^{\prime}, K_{X^{\prime}}+E^{\prime}-D^{\prime}\right) \\
& =\kappa\left(X^{\prime}, f^{*}\left(K_{X}-D\right)\right) \\
& =\kappa\left(X, K_{X}-D\right)=-\infty
\end{aligned}
$$

by the antiampleness of $K_{X}-D$. Hence, $h^{2}\left(X^{\prime}, D^{\prime}\right)=0$. Then, by the Riemann-Roch theorem, we obtain

$$
-h^{1}\left(X^{\prime}, D^{\prime}\right)=\chi\left(\mathcal{O}_{X^{\prime}}\right)+\frac{1}{2} D^{\prime} \cdot\left(D^{\prime}-K_{X^{\prime}}\right)=\chi\left(\mathcal{O}_{X^{\prime}}\right)
$$

because $D^{\prime}=f^{*} D \equiv 0$. This shows that

$$
0 \geq-h^{1}\left(X^{\prime}, D^{\prime}\right)=1-h^{1}\left(X^{\prime}, \mathcal{O}_{X^{\prime}}\right) .
$$

Thus, we obtain $h^{1}\left(X^{\prime}, \mathcal{O}_{X}^{\prime}\right) \geq 1$, and this means that $X^{\prime}$ is an irrational ruled surface. Let $\pi: X^{\prime} \rightarrow B$ be its ruling. Here, if $k=\overline{\mathbb{F}}_{p}$, then $D$ is a torsion by Corollary 2.4. Hence, we consider the case $k \neq \overline{\mathbb{F}}_{p}$. Then we can apply Theorem 3.20, and all $f$-exceptional curves are $\pi$-vertical. This shows that $\pi$ factors through $X^{\prime} \rightarrow X \rightarrow B$. A curve in a fiber has nonpositive self-intersection number. But this is a contradiction because each curve in $X$ is ample. This completes the proof of the case $C^{2}>0$.

Proof of the case where $C^{2}=0$. First let us prove that $\rho(X)=2$. It is sufficient to show that, for an arbitrary divisor $F$, if $F \cdot C=F \cdot\left(K_{X}+\Delta\right)=0$, then $F \equiv 0$. We need the following lemma.

Lemma 3.22. If $D_{1}, D_{2} \in C^{\perp}=\{D \mid D$ is a divisor and $D \cdot C=0\}$, then $D_{1} \cdot D_{2}=0$.

This proof is essentially due to [Mo2, Lemma 3.29].

Proof of Lemma 3.22. We consider the quadratic form $Q: C_{\mathbb{R}}^{\perp} \rightarrow \mathbb{R}$. Here we consider $C_{\mathbb{R}}^{\perp}$ as a subvector space of the numerical equivalence classes of $\mathbb{R}$-divisors, and $Q$ is defined by the self-intersection. We want to prove that $Q$ is identically 0 . Take a nef divisor $G$ such that $\overline{N E}(X) \cap G^{\perp}=\mathbb{R}_{\geq 0}[C]$. By the nefness of $G$, we obtain $G^{2} \geq 0$. But $G^{2}$ must be 0 because $G^{2}>0$ shows that $G$ is nef and big. Then, by $G \cdot C=0$, we obtain $C^{2}<0$, a contradiction. This shows that $Q$ is 0 in a nonempty dense subset of an open subset in $C_{\mathbb{R}}^{\perp}$ by the cone theorem. Therefore, $Q$ must be identically 0 . 
Since $F \in C^{\perp}$, we obtain $D \cdot F=0$ for any divisor $D \in C^{\perp}$. The $\mathbb{R}$ subvector-space $C_{\mathbb{R}}^{\perp}$ in numerical classes of divisors has codimension 1 . Take its basis $D_{1}, \ldots, D_{\rho-1}$. Then we get the basis $D_{1}, \ldots, D_{\rho-1}, K_{X}+\Delta$ of the whole space. Indeed, by $C \cdot\left(K_{X}+\Delta\right) \neq 0$, these vectors are linearly independent. Since $F \cdot D_{1}=\cdots=F \cdot D_{\rho-1}=F \cdot\left(K_{X}+\Delta\right)=0$, we get $F \equiv 0$. Thus, we obtain $\rho(X)=2$.

Next, let us prove that the divisor $C$ is semiample. By $C^{2}=0$ and $\left(K_{X}+\right.$ $\Delta) \cdot C<0$, we obtain $K_{X} \cdot C<0$. Let $f: X^{\prime} \rightarrow X$ be a resolution. By

$$
\begin{aligned}
\left(f^{*} C\right)^{2} & =f_{*}\left(f^{*} C\right) \cdot C=C \cdot C=0 \quad \text { and } \\
K_{X^{\prime}} \cdot f^{*} C & =f_{*}\left(K_{X^{\prime}}\right) \cdot C=K_{X} \cdot C<0,
\end{aligned}
$$

the Riemann-Roch theorem shows that $\kappa\left(X^{\prime}, f^{*} C\right) \geq 1$. Note that $h^{2}\left(X^{\prime}\right.$, $\left.n f^{*} C\right)=h^{0}\left(X^{\prime}, K_{X^{\prime}}-n f^{*} C\right)=0$ for all $n \gg 0$. Therefore, we get $\kappa(X, C)=$ $\kappa\left(X^{\prime}, f^{*} C\right) \geq 1$. Because $C^{2}=0$ implies that $\kappa(X, C)=1$, then by the following proposition, $C$ is a semiample divisor.

Proposition 3.23. Let $X$ be a projective normal surface, and let $L$ be a nef line bundle. If $\kappa(X, L)=1$, then $L$ is semiample.

Proof. For a proof, see [Fu, Theorem 4.1].

Hence, the complete linear system $|m C|$ induces a morphism $\phi_{R}: X \rightarrow Y$ to a smooth projective curve $Y$. This morphism satisfies (1), (2), and (4). We would like to show (3). Take a line bundle $L$ with $L \cdot C=0$. Since $\rho(X)=2$, we have $L \equiv q C$ for some rational number $q$. We take a large positive integer $s$ such that $q+s$ is positive. Then we have

$$
L+s C \equiv(q+s) C .
$$

By the same argument as above, we see that $L+s C$ is semiample. Then a sufficiently large multiple of $L+s C$ induces a morphism $\psi: X \rightarrow Z$ to a smooth projective curve $Z$. Moreover, since this morphism satisfies condition (1), we obtain the factorization

$$
\psi: X \stackrel{\phi_{R}}{\rightarrow} Y \stackrel{\sigma}{\rightarrow} Z
$$

with $\sigma_{*} \mathcal{O}_{Y}=\mathcal{O}_{Z}$. Since $Y$ and $Z$ are smooth projective curves, $\sigma$ must be an isomorphism. Then $n(L+s C)$ is a pullback of a line bundle on $Y$ for some positive integer $n$. Thus, we have (3). 
Before stating the proof of the case where $C^{2}<0$, we state a proposition on the contraction of $\mathbb{P}^{1}$.

Proposition 3.24. Let $X$ be a projective normal surface, and let $C$ be a curve in $X$ isomorphic to $\mathbb{P}^{1}$. Assume that $G$ is a nef and big line bundle on $X$ such that, for every curve $C^{\prime}$ in $X, G \cdot C^{\prime}=0$ if and only if $C^{\prime}=C$. Then, $G$ is semiample.

Proof. By Keel's result (Theorem 2.2), if $\left.G\right|_{C}$ is semiample, then $G$ is semiample. But, by $C=\mathbb{P}^{1}$, this is obvious.

Proof of the case where $C^{2}<0$. By $C \cdot\left(K_{X}+\Delta\right)<0$, we have $C \cdot\left(K_{X}+\right.$ $C)<0$. Therefore, by Theorem 3.19, we see that $C \simeq \mathbb{P}^{1}$. Let $G$ be a nef and big divisor such that for any curve $C^{\prime}, G \cdot C^{\prime}=0$ if and only if $C^{\prime}=C$. (The way to construct such a divisor $G$ is to let $H$ be an ample divisor and to let $G$ be the divisor such that $G=H+q C$ and $G \cdot C=0$ for rational number q.) Then, by Proposition 3.24, there exists $\phi_{R}$ satisfying (1) and (2). The remaining assertions (3), (4), and (5) hold from the following propositions.

First we prove (3). We generalize the setting a little for a later use.

Proposition 3.25 (Proof of (3)). Let $f: X \rightarrow Y$ be a proper birational morphism from a normal $\mathbb{Q}$-factorial surface $X$ to a normal surface $Y$. Assume that $C:=\operatorname{Ex}(f)$ is a proper irreducible curve and that $f(C)$ is one point. Let $L$ be a Cartier divisor on $X$ with $L \cdot C=0$. If $\left.L\right|_{C}$ is a torsion, then $n L=f^{*}\left(L_{Y}\right)$ for some Cartier divisor $L_{Y}$ on $Y$ and for some positive integer $n$.

Proof. We have the following.

STEP 1. In this step, we assume that $X$ and $Y$ are projective, and we prove the assertion.

Let $G$ be the pullback of an ample divisor. By Kodaira's lemma, $G=$ $A+E$, where $A$ is an ample $\mathbb{Q}$-divisor and $E$ is an effective $\mathbb{Q}$-divisor. By replacing $G$ by its suitable multiple, it is easy to see that we may assume that $E=q C$ for some $q \in \mathbb{Q}_{>0}$. Consider the divisor

$$
G^{\prime}=m G+L=(m A+L)+m q C
$$

for $m \gg 0$. Since $m A+L$ is ample for $m \gg 0$, we see that $G^{\prime} \cdot C^{\prime}>0$ for every curve $C^{\prime} \neq C$. On the other hand, we have

$$
G^{\prime} \cdot C=(m G+L) \cdot C=0 .
$$


Thus, for a sufficiently large integer $m \gg 0$, the Cartier divisor $G^{\prime}=m G+L$ is nef and big such that $G^{\prime} \cdot C^{\prime}=0$ if and only if $C^{\prime}=C$ for every curve $C^{\prime}$. Since $\left.L\right|_{C}$ is a torsion, $G^{\prime}=m G+L$ is semiample by Keel's result (Theorem 2.2). By Zariski's main theorem, $\left|n G^{\prime}\right|$ induces the same morphism as $f$ for some $n \in \mathbb{Z}_{>0}$. Thus, $n G^{\prime}=n m G+n L$ is a pullback of some line bundle on $Y$, and so is the difference $n L=n G^{\prime}-n m G$.

STEP 2. In this step, we assume that $Y$ is quasiprojective, and we prove the assertion.

Take a compactification $Y \subset \bar{Y}$ such that $\bar{Y}$ is projective and is smooth on $\bar{Y} \backslash Y$. We define $\bar{X}$ by patching $X$ and $\bar{Y}$ along $X \backslash C \simeq Y \backslash\{f(C)\}$. Then, $\bar{X}$ is projective because $\bar{X}$ is proper and $\mathbb{Q}$-factorial (see [F2, Lemma 2.2]). Thus, by Step 1, we obtain the required assertion.

STEP 3. In this step, we prove the assertion.

Let $f(C) \in Y_{0} \subset Y$ be an affine open subset, and let $X_{0}:=f^{-1}\left(Y_{0}\right)$. Let $\left.f\right|_{X_{0}}=: f_{0}$. Then, by Step 2, we obtain $\left.n L\right|_{X_{0}}=\left(f_{0}\right)^{*} L_{Y_{0}}$. Let $L_{Y}$ be the $\mathbb{Z}$-divisor on $Y$ such that $\left.L_{Y}\right|_{Y_{0}}=L_{Y_{0}}$ and such that $L_{Y}$ has no prime component contained in $Y \backslash Y_{0}$. Then, $L_{Y}$ is $\mathbb{Q}$-Cartier. Consider the prime decomposition

$$
L=\sum l_{i} C_{i}=\sum_{C_{i} \subset X \backslash X_{0}} l_{i} C_{i}+\sum_{C_{j} \not \subset X \backslash X_{0}} l_{j} C_{j} .
$$

We see that $n f^{*} L_{Y}=\sum_{C_{j} \not \subset X \backslash X_{0}} l_{j} C_{j}$. Since $\sum_{C_{j} \subset X \backslash X_{0}} l_{j} C_{j}$ is the pullback of some $\mathbb{Q}$-Cartier divisor, we obtain the assertion.

The condition (4) is an immediate corollary from (3). Thus, we prove (5).

Proposition 3.26 (Proof of (5)). Let $f: X \rightarrow Y$ be a proper birational morphism from a normal $\mathbb{Q}$-factorial surface $X$ to a normal surface $Y$. Assume that $C:=\operatorname{Ex}(f)$ is a proper irreducible curve and that $f(C)$ is one point. Assume the following condition.

(3) If $L$ is a Cartier divisor with $L \cdot C=0$, then $n L=\left(\phi_{R}\right)^{*} L_{Y}$ for some Cartier divisor $L_{Y}$ on $Y$ and for some positive integer $n$.

Then, $Y$ is $\mathbb{Q}$-factorial.

Proof. Let $E$ be a prime divisor on $Y$, and let $D$ be its proper transform. Since $C^{2}<0$, there exists a rational number $q$ such that $(D+q C) \cdot C=0$. By $(3)$, we have $n(D+q C)=f^{*}\left(L_{Y}\right)$ for some Cartier divisor $L_{Y}$. By operating $f_{*}$, we obtain the equality $n E=L_{Y}$ as Weil divisors. Therefore, $E$ is Q-Cartier. 
Since we have the cone theorem and the contraction theorem, we obtain the minimal model program for $\mathbb{Q}$-factorial surfaces with boundaries.

Theorem 3.27 (Minimal model program). Let $X$ be a projective normal $\mathbb{Q}$-factorial surface, and let $\Delta$ be an $\mathbb{R}$-boundary. Then, there exists a sequence of projective birational morphisms

$$
(X, \Delta)=:\left(X_{0}, \Delta_{0}\right) \stackrel{\phi_{0}}{\rightarrow}\left(X_{1}, \Delta_{1}\right) \stackrel{\phi_{1}}{\rightarrow} \cdots \stackrel{\phi_{s-1}}{\rightarrow}\left(X_{s}, \Delta_{s}\right)=:\left(X^{\dagger}, \Delta^{\dagger}\right)
$$

where $\left(\phi_{i-1}\right)_{*}\left(\Delta_{i-1}\right)=: \Delta_{i}$, with the following properties.

(1) Each $X_{i}$ is a projective normal $\mathbb{Q}$-factorial surface.

(2) Each $\Delta_{i}$ is an $\mathbb{R}$-boundary.

(3) For each $i, \operatorname{Ex}\left(\phi_{i}\right)=: C_{i}$ is an irreducible curve such that

$$
\left(K_{X_{i}}+\Delta_{i}\right) \cdot C_{i}<0
$$

and such that $C_{i}$ generates an extremal ray.

(4) The pair $\left(X^{\dagger}, \Delta^{\dagger}\right)$ satisfies one of the following conditions:

(a) $K_{X^{\dagger}}+\Delta^{\dagger}$ is nef;

(b) there is a projective surjective morphism $\mu: X^{\dagger} \rightarrow Z$ to a smooth projective curve $Z$ such that $\mu_{*} \mathcal{O}_{X^{\dagger}}=\mathcal{O}_{Z},-\left(K_{X}^{\dagger}+\Delta^{\dagger}\right)$ is $\mu$-ample, and $\rho\left(X^{\dagger}\right)=2$;

(c) $-\left(K_{X^{\dagger}}+\Delta^{\dagger}\right)$ is ample, and $\rho\left(X^{\dagger}\right)=1$.

\subsection{Finite generation of canonical rings}

It is important to consider the finite generation of canonical rings, which is closely related to the minimal model program. In this section, we prove the following theorem.

THEOREM 3.28 (Finite generation theorem). Let $X$ be a projective normal $\mathbb{Q}$-factorial surface over $k$, and let $\Delta$ be a $\mathbb{Q}$-boundary. Then $R\left(X, K_{X}+\right.$ $\Delta):=\bigoplus_{m \geq 0} H^{0}\left(X,\left\llcorner m\left(K_{X}+\Delta\right)\right\lrcorner\right)$ is a finitely generated $k$-algebra.

Proof. Let us consider the Kodaira dimension $\kappa:=\kappa\left(X, K_{X}+\Delta\right)$. It is obvious for the case $\kappa=-\infty$ and the case $\kappa=0$. In particular, we may assume that $K_{X}+\Delta$ is effective. Then, by Theorem 3.27, we may assume that $K_{X}+\Delta$ is nef. The case $\kappa=1$ follows from Proposition 3.23. Therefore, we may assume that $\kappa=2$, that is, that $K_{X}+\Delta$ is nef and big. This case follows from Proposition 3.29. 
Proposition 3.29. Let $X$ be a projective normal $\mathbb{Q}$-factorial surface, and let $\Delta$ be a $\mathbb{Q}$-boundary. If $K_{X}+\Delta$ is nef and big, then $K_{X}+\Delta$ is semiample.

Proof. By Keel's result (Theorem 2.2), it is sufficient to prove that if

$$
E:=\bigcup_{C \cdot\left(K_{X}+\Delta\right)=0} C=C_{1} \cup \cdots \cup C_{r},
$$

then $\left.\left(K_{X}+\Delta\right)\right|_{E}$ is semiample. Let $C \subset E$. Then we have

$$
\left(K_{X}+C\right) \cdot C \leq\left(K_{X}+\Delta\right) \cdot C=0 .
$$

STEP 1. In this step, we reduce the proof to the case where, if $C \subset E$, then $\left(K_{X}+C\right) \cdot C=0$.

Assume that $C \subset E$ and that $\left(K_{X}+C\right) \cdot C<0$. Then $C$ is a $\left(K_{X}+\right.$ $C)$-negative extremal curve. Thus, by Theorem 3.21, we can contract $C$. Let $f: X \rightarrow Y$ be its contraction, and let $\Delta_{Y}:=f_{*}(\Delta)$. Then since $K_{X}+$ $\Delta=f^{*}\left(K_{Y}+\Delta_{Y}\right)$ and $Y$ is $\mathbb{Q}$-factorial, if we can prove that $K_{Y}+\Delta_{Y}$ is semiample, then $K_{X}+\Delta$ is semiample. We can repeat this procedure and obtain the desired reduction.

STEP 2. In this step, we prove that $E$ is a disjoint union of irreducible curves and that if $C \subset E$, then $\left.\left(K_{X}+\Delta\right)\right|_{C}=\left.\left(K_{X}+C\right)\right|_{C}$.

Let $C \subset E$. By Step 1, we have $\left(K_{X}+C\right) \cdot C=0$. Then, the inequality over Step 1 is an equality. Thus, $C \subset \operatorname{Supp} \Delta$, and $C$ is disjoint from any other component of $\Delta$.

By Step 2, it is sufficient to prove that, if $\left(K_{X}+C\right) \cdot C=0$, then $\left(K_{X}+\right.$ $C)\left.\right|_{C}$ is semiample. This is satisfied by Theorem 3.19 .

\subsection{Abundance theorem $\left(k \neq \overline{\mathbb{F}}_{p}\right)$}

In this section, we prove the abundance theorem for $\mathbb{Q}$-factorial surfaces with $\mathbb{Q}$-boundary over $k \neq \overline{\mathbb{F}}_{p}$. The case where $k=\overline{\mathbb{F}}_{p}$ will be treated in Section 4 . In the case where $\kappa\left(X, K_{X}+\Delta\right)=0$, we give a proof of the abundance theorem which does not depend on the characteristic of the base field $k$ (see Theorem 3.34).

First we present the following nonvanishing theorem.

THEOREM 3.30 (Nonvanishing theorem). Let $k$ be an algebraically closed field of arbitrary characteristic. Let $X$ be a projective normal $\mathbb{Q}$-factorial surface over $k$, and let $\Delta$ be a $\mathbb{Q}$-boundary. If $k$ is not the algebraic closure of a finite field and if $K_{X}+\Delta$ is pseudoeffective, then $\kappa\left(X, K_{X}+\Delta\right) \geq 0$. 
Proof. For a proof, see [F2, Theorem 5.1, Lemma 5.2]. Note that in this article, instead of [F2, Lemma 5.2], we use Theorem 3.20 of this paper.

Before the proof of the abundance theorem, we present the definition of indecomposable curves of canonical type in the sense of [Mu2, p. 330].

DEFINITION 3.31. Let $k$ be an algebraically closed field of arbitrary characteristic. Let $X$ be a smooth projective surface over $k$, and let $Y=\sum n_{i} E_{i}$ be an effective divisor with $n_{i} \in \mathbb{Z}_{>0}$. We say that $Y$ is an indecomposable curve of canonical type if $Y \neq 0, K_{X} \cdot E_{i}=Y \cdot E_{i}=0$ for all $i, \operatorname{Supp} Y$ is connected, and $\operatorname{gcd}\left(n_{i}\right)=1$.

We present criteria for the movability of indecomposable curves of canonical type.

Proposition 3.32. Let $k$ be an algebraically closed field of arbitrary characteristic. Let $X$ be a smooth projective surface over $k$, and let $Y$ be an indecomposable curve of canonical type in $X$. Assume that one of the following assertions holds:

(1) $\operatorname{char} k=p>0$;

(2) $H^{1}\left(X, \mathcal{O}_{X}\right)=0$.

If $\mathcal{O}_{Y}(Y)$ is a torsion, then $\kappa(X, Y)=1$.

The proof of (2) is very similar to that of [To, Theorem 2.1].

Proof of Proposition 3.32. (1) For a proof, see [M, lemma on p. 682].

(2) Assume that $H^{1}\left(X, \mathcal{O}_{X}\right)=0$. Let $m$ be the order of $\mathcal{O}_{Y}(Y)$, and let $a$ be an integer with $1 \leq a \leq m-1$. The case where $\mathcal{O}_{Y}(Y)=\mathcal{O}_{Y}$ is easy, so we exclude this case and can assume that $m \geq 2$. Let us consider the following exact sequence:

$$
H^{0}\left(Y, \mathcal{O}_{Y}(a Y)\right) \rightarrow H^{1}(X,(a-1) Y) \rightarrow H^{1}(X, a Y) \rightarrow H^{1}\left(Y, \mathcal{O}_{Y}(a Y)\right)
$$

By Serre duality and [Mu2, Corollary 1 on p. 333], we obtain

$$
h^{1}\left(Y, \mathcal{O}_{Y}(a Y)\right)=h^{0}\left(Y, \mathcal{O}_{Y}\left(K_{Y}-a Y\right)\right)=h^{0}\left(Y, \mathcal{O}_{Y}(-a Y)\right) .
$$

The choice of $a$ shows that

$$
h^{0}\left(Y, \mathcal{O}_{Y}(a Y)\right)=h^{0}\left(Y, \mathcal{O}_{Y}(-a Y)\right)=0
$$


Indeed, suppose the contrary; for example, suppose that $h^{0}\left(Y,\left.a Y\right|_{Y}\right) \neq 0$. Then we have $\mathcal{O}_{Y}(a Y)=\mathcal{O}_{Y}$ by [Mu2, lemma on p. 332]. This is a contradiction. Therefore, we get

$$
0=h^{1}\left(X, \mathcal{O}_{X}\right)=h^{1}(X, Y)=\cdots=h^{1}(X,(m-1) Y) .
$$

This leads to the following exact sequence:

$$
0 \rightarrow H^{0}(X,(m-1) Y) \rightarrow H^{0}(X, m Y) \rightarrow H^{0}\left(Y, \mathcal{O}_{Y}\right) \rightarrow 0
$$

Thus, $Y$ is an effective semiample divisor on $X$, and $Y^{2}=0$. This shows that $\kappa(X, Y)=1$.

Now, we prove the abundance theorem.

THEOREM 3.33 (Abundance theorem). Let $k$ be an algebraically closed field of positive characteristic. Let $X$ be a projective normal $\mathbb{Q}$-factorial surface over $k$, and let $\Delta$ be a $\mathbb{Q}$-boundary. If $k$ is not the algebraic closure of a finite field and $K_{X}+\Delta$ is nef, then $K_{X}+\Delta$ is semiample.

Proof. By Theorem 3.30, we may assume that $\kappa\left(K_{X}+\Delta\right) \geq 0$. Moreover, we may assume that $\kappa\left(K_{X}+\Delta\right)=0$ by Propositions 3.23 and 3.29. Thus, it is sufficient to prove the following theorem.

THEOREM 3.34. Let $k$ be an algebraically closed field of arbitrary characteristic. Let $X$ be a projective normal $\mathbb{Q}$-factorial surface over $k$, and let $\Delta$ be a $\mathbb{Q}$-boundary. If $k$ is not the algebraic closure of a finite field, $K_{X}+\Delta$ is nef, and $\kappa\left(X, K_{X}+\Delta\right)=0$, then $K_{X}+\Delta \sim_{\mathbb{Q}} 0$.

This proof is very similar to that of [F2, Theorem 6.1] and uses many of the techniques in [Fu, Section 5].

Proof of Theorem 3.34. Let $f: V \rightarrow X$ be the minimal resolution. We set $K_{V}+\Delta_{V}=f^{*}\left(K_{X}+\Delta\right)$. We note that $\Delta_{V}$ is effective. It is sufficient to see that $K_{V}+\Delta_{V} \sim_{\mathbb{Q}} 0$. Let

$$
\varphi: V=: V_{0} \stackrel{\varphi_{0}}{\rightarrow} V_{1} \stackrel{\varphi_{1}}{\rightarrow} \cdots \stackrel{\varphi_{k-1}}{\rightarrow} V_{k}=: S
$$

be a sequence of blowdowns such that

(1) $\varphi_{i}$ is a blowdown of a $(-1)$-curve $C_{i}$ on $V_{i}$,

(2) $\Delta_{V_{i+1}}=\varphi_{i *} \Delta_{V_{i}}$, and

(3) $\left(K_{V_{i}}+\Delta_{V_{i}}\right) \cdot C_{i}=0$, 
for every $i$. We can assume that there are no $(-1)$-curves $C$ on $S$ with $\left(K_{S}+\Delta_{S}\right) \cdot C=0$. We note that $K_{V}+\Delta_{V}=\varphi^{*}\left(K_{S}+\Delta_{S}\right)$. It is sufficient to show that $K_{S}+\Delta_{S} \sim_{\mathbb{Q}} 0$. Since $\kappa\left(S, K_{S}+\Delta_{S}\right)=0$, there is a member $Z$ of $\left|m\left(K_{S}+\Delta_{S}\right)\right|$ for some positive integer $m$. Then, for every positive integer $t, t Z$ is the unique member of $\left|\operatorname{tm}\left(K_{S}+\Delta_{S}\right)\right|$. We will derive a contradiction assuming that $Z \neq 0$.

STEP 1. In this step, we prove that for each prime component $Z_{i}$ of $Z$, we have

$$
K_{S} \cdot Z_{i}=\Delta_{S} \cdot Z_{i}=Z \cdot Z_{i}=0
$$

Since $\left(K_{S}+\Delta_{S}\right) \cdot Z=m\left(K_{S}+\Delta_{S}\right)^{2}=0$ and $\left(K_{S}+\Delta_{S}\right)$ is nef, $\left(K_{S}+\Delta_{S}\right)$. $Z_{i}=0$ for all $i$. This means that

$$
Z \cdot Z_{i}=0
$$

and that $Z_{i}^{2} \leq 0$. Now, we prove that $K_{S} \cdot Z_{i} \geq 0$ for every $i$. If $K_{S} \cdot Z_{i}<0$, then we obtain $\left(K_{S}+Z_{i}\right) \cdot Z_{i}<0$ and $Z_{i} \cong \mathbb{P}^{1}$. If $Z_{i}^{2} \geq 0$, then we obtain $\kappa(S, Z) \geq \kappa\left(S, Z_{i}\right)>0$. This contradicts $\kappa\left(S, K_{S}+\Delta_{S}\right)=\kappa(S, Z)=0$. If $Z_{i}^{2}<0$, then $Z_{i}$ is a $(-1)$-curve with $\left(K_{S}+\Delta_{S}\right) \cdot Z_{i}=0$. This contradicts the definition of $S$. Regardless, we have $K_{S} \cdot Z_{i} \geq 0$ for every $i$. This implies that $K_{S} \cdot Z=K_{S} \cdot m\left(K_{S}+\Delta_{S}\right) \geq 0$. The nefness of $K_{S}+\Delta_{S}$ shows that $\left(K_{S}+\Delta_{S}\right) \cdot \Delta_{S} \geq 0$. By $\left(K_{S}+\Delta_{S}\right)^{2}=0$, we see that $\left(K_{S}+\Delta_{S}\right) \cdot K_{S}=\left(K_{S}+\right.$ $\left.\Delta_{S}\right) \cdot \Delta_{S}=0$. This is equivalent to $Z \cdot K_{S}=Z \cdot \Delta_{S}=0$. Since $K_{S} \cdot Z_{i} \geq 0$, we see that

$$
K_{S} \cdot Z_{i}=\Delta_{S} \cdot Z_{i}=0
$$

SteP 2. We can decompose $Z$ into the connected components as follows:

$$
Z=\sum_{i=1}^{r} \mu_{i} Y_{i}
$$

where $\mu_{i} Y_{i}$ is a connected component of $Z$ such that $\mu_{i}$ is the greatest common divisor of the coefficients of prime components of $Y_{i}$ in $Z$ for every $i$. Then we see that, for every $i$, each $Y_{i}$ is an indecomposable curve of canonical type by Step 1 . We obtain $\omega_{Y_{i}} \simeq \mathcal{O}_{Y_{i}}$ by [Mu2, Corollary 1 on p. 333].

STEP 3. In this step, we assume that $\kappa\left(S, K_{S}\right) \geq 0$ and prove the assertion. Since

$$
0 \leq \kappa\left(S, K_{S}\right) \leq \kappa\left(S, K_{S}+\Delta_{S}\right)=0
$$

we obtain $\kappa\left(S, K_{S}\right)=0$. Let us prove that $S$ is a minimal surface. 
Suppose the contrary; that is, suppose that there exists a $(-1)$-curve $E$. Then we have the contraction $g: S \rightarrow S^{\prime}$ of $E$, and we obtain a morphism

$$
S \stackrel{g}{\rightarrow} S^{\prime} \stackrel{h}{\rightarrow} S_{\min }
$$

to a minimal surface $S_{\min }$. Since $0=\kappa\left(S, K_{S}\right)=\kappa\left(S_{\min }, K_{S_{\min }}\right)$, we see that $K_{S_{\min }} \sim_{\mathbb{Q}} 0$. Because

$$
K_{S}=g^{*} K_{S^{\prime}}+E=g^{*}\left(h^{*}\left(K_{S_{\min }}\right)+(\text { effective divisor })\right)+E,
$$

we see that $K_{S} \sim_{\mathbb{Q}}($ effective divisor $)+E$. This means that

$$
n Z \sim n m\left(K_{S}+\Delta_{S}\right) \sim(\text { effective divisor })+n m E+n m \Delta_{S}
$$

for some $n \in \mathbb{Z}_{>0}$. Since $\kappa(S, Z)=0$,

$$
n Z=(\text { effective divisor })+n m E+n m \Delta_{S}
$$

as Weil divisors. In particular, we have $E \subset \operatorname{Supp} Z$ and $E=Z_{i}$ for some $i$. This implies that $Z_{i} \cdot\left(K_{S}+\Delta_{S}\right)=0$ and that $Z_{i}$ is a $(-1)$-curve, a contradiction to the construction of $S$. Therefore, $S$ is minimal.

Then we obtain the contradiction

$$
\kappa\left(S, K_{S}+\Delta_{S}\right)=\kappa(S, Z) \geq \kappa\left(S, Y_{i}\right) \geq 1
$$

from the known result $\kappa\left(S, Y_{i}\right) \geq 1$ (see, e.g., [Bă1, Theorem 7.11]).

SteP 4. By Step 3, we may assume that $\kappa\left(S, K_{S}\right)=-\infty$. In Steps 5 and 6 , we assume that $S$ is rational and prove the assertion. In Steps 712 , we assume that $S$ is irrational and we prove the assertion. Note that since $\kappa(X, Z)=0$, in order to derive a contradiction, we want to prove that $\kappa\left(X, Y_{i}\right) \geq 1$ for some $i$.

We assume that $S$ is rational.

STEP 5. In this step, we prove that

$$
\Delta_{S}=\sum y_{i} Y_{i} \quad \text { and } \quad y_{i}>1
$$

We fix $i$. By $H^{1}\left(S, \mathcal{O}_{S}\left(K_{S}\right)\right)=0$ and the exact sequence

$$
0 \rightarrow \mathcal{O}_{S}\left(K_{S}\right) \rightarrow \mathcal{O}_{S}\left(K_{S}+Y_{i}\right) \rightarrow \omega_{Y_{i}} \rightarrow 0
$$


we obtain the surjection

$$
H^{0}\left(S, \mathcal{O}_{S}\left(K_{S}+Y_{i}\right)\right) \rightarrow H^{0}\left(Y_{i}, \omega_{Y_{i}}\right) \simeq H^{0}\left(Y_{i}, \mathcal{O}_{Y_{i}}\right) .
$$

Thus, there exists $W_{i} \in\left|K_{S}+Y_{i}\right|$ such that $W_{i}$ has no components of $Y_{i}$. For $\tilde{Z}_{i}=Z-\mu_{i} Y_{i}$, we obtain the equation

$$
m \mu_{i} W_{i}+m \mu_{i} \Delta_{S}+m \tilde{Z}_{i}=\left(\mu_{i}+m\right) Z .
$$

Note that this equality holds as Weil divisors because $\kappa(S, Z)=0$. From this equation, Supp $\Delta_{S} \subset \operatorname{Supp} Z$. Since $W_{i}$ and $\tilde{Z}_{i}$ are free from the components of $Y_{i}$, we have $\Delta_{S}=\sum\left(\left(\mu_{i}+m\right) / m\right) Y_{i}$. We set $y_{i}:=\left(\mu_{i}+m\right) / m>1$.

We fix $i$, and we denote $Y$ instead of $Y_{i}$.

SteP 6. In this step, we prove the desired assertion. By Proposition 3.32(2), it is sufficient to prove that $\mathcal{O}_{Y}(a Y) \simeq \mathcal{O}_{Y}$ for some positive integer $a$. We set $Y_{(k)}:=Y$ and construct $Y_{(j)}$ inductively. It is easy to see that $\varphi_{j}: V_{j} \rightarrow V_{j+1}$ is the blowup at $P_{j+1}$ with mult $P_{j+1} \Delta_{V_{j+1}} \geq 1$ for every $j$ since $\Delta_{V_{j}}$ is effective. If mult $_{P_{j+1}} Y_{(j+1)}=0$, then we set $Y_{(j)}=\varphi_{j}^{*} Y_{(j+1)}$. If $\operatorname{mult}_{P_{j+1}} Y_{(j+1)}>$ 0 , then we set $Y_{(j)}=\varphi_{j}^{*} Y_{(j+1)}-C_{j}$, where $C_{j}$ is the exceptional curve of $\varphi_{j}$. Thus, we obtain $Y_{(0)}$ on $V_{0}=V$. Note that $\operatorname{mult}_{P} \Delta_{V_{j+1}}>\operatorname{mult}_{P} Y_{(j+1)}$ for every $P \in \operatorname{Supp} Y_{(j+1)}$ by Step 5 and the above inductive construction. Moreover, since mult $_{P} Y_{(j+1)} \in \mathbb{Z}$, we see that $Y_{(0)}$ is effective and that $\operatorname{Supp} Y_{(0)} \subset$ $\operatorname{Supp} \Delta_{V}^{>1}$ where, for the prime decomposition $\Delta_{V}=\sum \delta_{l} \Delta_{V, l}$, we define $\Delta_{V}^{>1}:=\sum_{\delta_{l}>1} \delta_{l} \Delta_{V, l}$. Then, we have $\varphi_{j *} \mathcal{O}_{Y_{(j)}} \simeq \mathcal{O}_{Y_{(j+1)}}$ for every $j$. Indeed, $\varphi_{j *} \mathcal{O}_{V_{j}}\left(-Y_{(j)}\right) \simeq \mathcal{O}_{V_{j+1}}\left(-Y_{(j+1)}\right)$ and $R^{1} \varphi_{j *} \mathcal{O}_{V_{j}}\left(-Y_{(j)}\right)=0$ for every $j$. See the following commutative diagram:

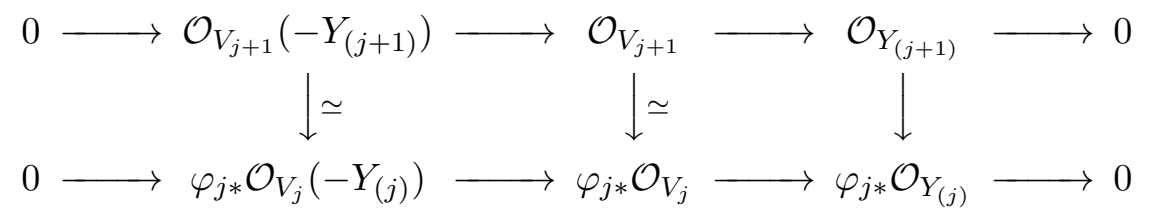

Therefore, we obtain $\varphi_{*} \mathcal{O}_{Y_{(0)}} \simeq \mathcal{O}_{Y}$. Since $\operatorname{Supp} Y_{(0)} \subset \operatorname{Supp} \Delta_{V}^{>1}$, we see that $Y_{(0)}$ is $f$-exceptional. Since $K_{V}+\Delta_{V}=f^{*}\left(K_{X}+\Delta\right)$, we obtain $\mathcal{O}_{Y_{(0)}}\left(b\left(K_{V}+\right.\right.$ $\left.\left.\Delta_{V}\right)\right) \simeq \mathcal{O}_{Y_{(0)}}$ for some positive divisible integer $b$. Thus,

$$
\mathcal{O}_{Y}\left(b\left(K_{S}+\Delta_{S}\right)\right) \simeq \varphi_{*} \mathcal{O}_{Y_{(0)}}\left(b\left(K_{V}+\Delta_{V}\right)\right) \simeq \varphi_{*} \mathcal{O}_{Y_{(0)}} \simeq \mathcal{O}_{Y}
$$

This means that

$$
\mathcal{O}_{Y}(\mu b Y) \simeq \mathcal{O}_{Y}(b Z) \simeq \mathcal{O}_{Y}\left(b m\left(K_{S}+\Delta_{S}\right)\right) \simeq \mathcal{O}_{Y}
$$

This completes the proof of the rational case. 
We assume that $S$ is an irrational ruled surface. Let $\pi: S \rightarrow B$ be its ruling, and let $F$ be one of its smooth fibers.

STEP 7. In this step, we prove that each connected component $\mu Y$ of $Z$ satisfies $F \cdot Y>0$.

We assume that $F \cdot Y=0$ and derive a contradiction. Since $Y$ is connected, $Y$ is contained in some fiber $F_{0}$. Then we have the equality

$$
F_{0}=y Y+Y^{\prime}
$$

for some effective $\mathbb{Q}$-divisor $Y^{\prime}$ with $\operatorname{Supp} Y \not \subset \operatorname{Supp} Y^{\prime}$ and for some positive rational number $y$. By $K_{S} \cdot F_{0}=-2$ and $K_{S} \cdot Y=0$, it is sufficient to prove that $Y^{\prime}=0$. Thus, assume that $Y^{\prime} \neq 0$. Take a prime component $Y_{(1)}$ of $Y$ which is not a component of $Y^{\prime}$. The equalities $F_{0} \cdot Y_{(1)}=Y \cdot Y_{(1)}=$ 0 show that $Y^{\prime} \cdot Y_{(1)}=0$. Thus, if $Y_{(2)}$ is a prime component of $Y$ such that $Y_{(1)} \cap Y_{(2)} \neq \emptyset$, then $Y_{(2)}$ is not a component of $Y^{\prime}$. By repeating this procedure, we see that $Y_{(i)}$ is not a prime component of $Y^{\prime}$ for each prime component $Y_{(i)}$ of $Y$. Since $Y^{\prime} \neq 0$, there exists a prime component $Y_{(j)}$ of $Y$ with $Y_{(j)} \cap Y^{\prime} \neq \emptyset$. This leads to the contradiction

$$
F_{0} \cdot Y_{(j)}=Y \cdot Y_{(j)}=0 \quad \text { and } \quad Y^{\prime} \cdot Y_{(j)} \neq 0 .
$$

STEP 8. In this step, we prove that both $B$ and $Y$ are elliptic curves. In particular, $Y^{2}=0$.

By Step 7, $Y$ has a prime component $Y_{(0)}$ with $\pi\left(Y_{(0)}\right)=B$. Because $\left(K_{S}+Y_{(0)}\right) \cdot Y_{(0)} \leq 0$ by Step $1, Y_{(0)}$ is a rational curve or an elliptic curve. But, since $B$ is irrational and $\pi\left(Y_{(0)}\right)=B, Y_{(0)}$ must be an elliptic curve. Then, $B$ is also an elliptic curve. Moreover, if an indecomposable curve of canonical type $Y$ is reducible, then every prime component of $Y$ must be $\mathbb{P}^{1}$.

Indeed, for every prime component $Y_{(i)}$, we have $\left(K_{S}+Y_{(i)}\right) \cdot Y_{(i)} \leq 0$. Assume that a prime component $Y_{(0)}$ satisfies $\left(K_{S}+Y_{(0)}\right) \cdot Y_{(0)}=0$. Then, by $K_{S} \cdot Y_{(0)}=0$, we have $Y_{(0)}^{2}=0$. Since $Y \cdot Y_{(0)}=0$, we obtain $Y_{(i)} \cdot Y_{(0)}=0$ for every prime component $Y_{(i)}$. Because $Y$ is connected, $Y$ must be irreducible.

STEP 9. In this step, we prove that the coefficient $\delta$ of $Y$ in $\Delta_{S}$ satisfies $0 \leq \delta \leq 1$

Assume the contrary; that is, we assume that $\delta>1$ and derive a contradiction. Take the proper transform $Y_{V}$ of $Y$ in $V$. We see that the coefficient of $Y_{V}$ in $\Delta_{V}$ is $\delta$. Then $Y_{V}$ is contracted by $f$ because of the assumption of the boundary. Since $X$ is $\mathbb{Q}$-factorial, we can apply Theorem 3.20, and this is a contradiction. 
STEP 10. In this step, we prove that $\mathcal{O}_{Y}(Y)$ is a torsion.

By Step 1, we have $Y \cdot\left(\Delta_{S}-\delta Y\right)=0$. This means that $\operatorname{Supp} Y \cap$ $\operatorname{Supp}\left(\Delta_{S}-\delta Y\right)=\emptyset$. Thus, in $\operatorname{Pic} Y$, we obtain

$$
\mu Y=Z=m\left(K_{S}+\Delta_{S}\right)=m\left(K_{S}+\delta Y\right)=m(-Y+\delta Y) .
$$

Therefore, we have $(m(1-\delta)+\mu) Y=0$ in Pic $Y$. By $m(1-\delta)+\mu>m(1-$ $\delta) \geq 0, \mathcal{O}_{Y}(Y)$ must be a torsion.

STEP 11. Let $r$ be the order of the torsion $\mathcal{O}_{Y}(Y)$. In this step, we prove that

$$
H^{1}\left(S, \mathcal{O}_{S}\left(K_{S}+t Y\right)\right)=0
$$

for $1 \leq t \leq r$ by induction.

Let us consider the exact sequence

$$
0 \rightarrow \mathcal{O}_{S}\left(K_{S}\right) \rightarrow \mathcal{O}_{S}\left(K_{S}+Y\right) \rightarrow \omega_{Y} \rightarrow 0
$$

If the induced map

$$
H^{0}\left(S, \mathcal{O}_{S}\left(K_{S}+Y\right)\right) \rightarrow H^{0}\left(Y, \omega_{Y}\right)=k
$$

is surjective, we get a contradiction by the same argument as in Step 5 . Therefore, this map is 0 . Then, the injective map

$$
k=H^{0}\left(Y, \omega_{Y}\right) \rightarrow H^{1}\left(S, \mathcal{O}_{S}\left(K_{S}\right)\right)=k
$$

is bijective. This means that the map

$$
H^{1}\left(S, \mathcal{O}_{S}\left(K_{S}+Y\right)\right) \rightarrow H^{1}\left(Y, \omega_{Y}\right)
$$

is injective. On the other hand, we have $h^{2}\left(S, \mathcal{O}_{S}\left(K_{S}+Y\right)\right)=0$ by Serre duality. Then we obtain the surjective map

$$
H^{1}\left(Y, \omega_{Y}\right) \rightarrow H^{2}\left(S, \mathcal{O}_{S}\left(K_{S}\right)\right)
$$

But this is bijective by Serre duality. Therefore, we obtain

$$
H^{1}\left(S, \mathcal{O}_{S}\left(K_{S}+Y\right)\right)=0
$$

and this proves the case where $t=1$. When $1<t \leq r$, we have the exact sequence

$$
H^{1}\left(S, K_{S}+(t-1) Y\right) \rightarrow H^{1}\left(S, K_{S}+t Y\right) \rightarrow H^{1}\left(Y, K_{S}+t Y\right) .
$$

By the induction hypothesis, we have $H^{1}\left(S, K_{S}+(t-1) Y\right)=0$. Moreover, we obtain $h^{1}\left(Y, \mathcal{O}_{Y}\left(K_{S}+t Y\right)\right)=h^{0}\left(Y, \mathcal{O}_{Y}(-(t-1) Y)\right)=0$ since $r$ is the order of $\mathcal{O}_{Y}(Y)$. Thus, we see that $H^{1}\left(S, K_{S}+t Y\right)=0$ for $1 \leq t \leq r$. 
SteP 12. By Step 11, we obtain a surjection

$$
H^{0}\left(S, \mathcal{O}_{S}\left(K_{S}+(r+1) Y\right)\right) \rightarrow H^{0}\left(Y, \mathcal{O}_{Y}\left(K_{S}+(r+1) Y\right)\right)
$$

By $\mathcal{O}_{Y}\left(K_{S}+(r+1) Y\right)=\mathcal{O}_{Y}\left(K_{Y}+r Y\right)=\mathcal{O}_{Y}$, there exists an effective member $W \in\left|K_{S}+(r+1) Y\right|$ free from $Y$. Set $\tilde{Z}:=Z-\mu Y$. We obtain the equation

$$
\begin{aligned}
\mu Z+(r+1) m Z & =\mu m\left(K_{S}+\Delta_{S}\right)+(r+1) m(\mu Y+\tilde{Z}) \\
& =\mu m\left(K_{S}+(r+1) Y\right)+\mu m \Delta_{S}+(r+1) m \tilde{Z} \\
& =\mu m W+\mu m \Delta_{S}+(r+1) m \tilde{Z}
\end{aligned}
$$

as Weil divisors. By considering the coefficients of $Y$ in both sides, we obtain

$$
(\mu+(r+1) m) \mu=\mu m \delta .
$$

But these two numbers are different by $0 \leq \delta \leq 1$. This is a contradiction.

REMARK 3.35. If char $k>0$, then we do not need Steps 11 and 12 in the proof of Theorem 3.34 by using Proposition 3.32(1).

\subsection{Abundance theorem for $\mathbb{R}$-divisors $\left(k \neq \overline{\mathbb{F}}_{p}\right)$}

In this section, we establish the abundance theorem in the case where $\Delta$ is an $\mathbb{R}$-boundary. We fix the following notation.

Notation 3.36. Let $X$ be a projective normal $\mathbb{Q}$-factorial surface. We fix prime divisors $\Lambda_{1}, \ldots, \Lambda_{s}$ and a positive integer $\lambda \in \mathbb{Z}_{>0}$. Let

$$
\mathcal{L}:=\left\{B \in \sum \mathbb{R} \Lambda_{i} \mid 0 \leq B \leq \lambda \sum \Lambda_{i}\right\} .
$$

Let

$$
M(X, \mathcal{L}):=\max \left(\{3\} \cup\left\{-\left(K_{X}+\lambda \Lambda_{i}\right) \cdot \Lambda_{i}\right\}\right),
$$

where $i$ ranges over $1 \leq i \leq s$.

Lemma 3.37 and Proposition 3.39 play key roles in this section. The arguments are extracted from [Bi, Section 3].

LEMMA 3.37. If $R$ is an extremal ray of $\overline{N E}(X)$ spanned by a curve, then there exists a curve $C$ such that $R=\mathbb{R}_{\geq 0}[C]$ and $-\left(K_{X}+B\right) \cdot C \leq M(X, \mathcal{L})$ for all $B \in \mathcal{L}$. 
Proof. Let $H$ be an ample line bundle on $X$. Take a curve $C$ with

$$
R=\mathbb{R}_{\geq 0}[C] \quad \text { and } \quad H \cdot C=\min \{H \cdot D\},
$$

where $D$ ranges over curves generating $R$. We want to prove that $C$ satisfies the desired condition. Set $B \in \mathcal{L}$. If $-\left(K_{X}+B\right) \cdot C \leq 0$, then there is nothing to prove. Thus, we may assume that $-\left(K_{X}+B\right) \cdot C>0$. This means that $R$ is a $\left(K_{X}+B\right)$-negative extremal ray. Then, by Theorem 3.13 and Remark 3.14, there exists a curve $C^{\prime}$ such that $R=\mathbb{R}_{\geq 0}\left[C^{\prime}\right]$ and

$$
-\left(K_{X}+B\right) \cdot C^{\prime} \leq L(X, B)=\max \left(\{3\} \cup\left\{-\left(K_{X}+B\right) \cdot \Lambda_{\mu}\right\}\right),
$$

where $\Lambda_{\mu}$ ranges over the prime components $\Lambda_{\mu}$ of $B$ with $\Lambda_{\mu}^{2}<0$. Here, by the definition of $M(X, \mathcal{L})$, we have $L(X, B) \leq M(X, \mathcal{L})$. Thus, we obtain

$$
-\left(K_{X}+B\right) \cdot C^{\prime} \leq M(X, \mathcal{L}) .
$$

By

$$
\frac{-\left(K_{X}+B\right) \cdot C}{H \cdot C}=\frac{-\left(K_{X}+B\right) \cdot C^{\prime}}{H \cdot C^{\prime}}
$$

we have

$$
\begin{aligned}
-\left(K_{X}+B\right) \cdot C & =\left(-\left(K_{X}+B\right) \cdot C^{\prime}\right) \frac{H \cdot C}{H \cdot C^{\prime}} \\
& \leq-\left(K_{X}+B\right) \cdot C^{\prime} \\
& \leq M(X, \mathcal{L}) .
\end{aligned}
$$

This completes the proof.

Definition 3.38. For an $\mathbb{R}$-divisor $B \in \sum_{i=1}^{s} \mathbb{R} \Lambda_{i}$ and for its prime decomposition $B=\sum r_{i} \Lambda_{i}$, we define

$$
\|B\|:=\left(\sum\left|r_{i}\right|^{2}\right)^{1 / 2}
$$

where $\left|r_{i}\right|$ is the absolute value of $r_{i}$.

Proposition 3.39. Let $\Gamma$ be a $\mathbb{Q}$-divisor on $X$. Let $M$ be a positive real number.

(1) Let $\Delta \in \mathcal{L}$. Then there exists a positive real number $\epsilon$ depending on $X, \mathcal{L}, \Delta, \Gamma$, and $M$, which satisfies the following property: let $C$ be a curve on $X$ such that $-\left(K_{X}+\Gamma+B\right) \cdot C \leq M$ for all $B \in \mathcal{L}$. If $\left(K_{X}+\Gamma+\Delta\right) \cdot C>0$, then $\left(K_{X}+\Gamma+\Delta\right) \cdot C>\epsilon$. 
(2) Let $\Delta \in \mathcal{L}$. Then, there exists a positive real number $\delta$, depending on $X$, $\mathcal{L}, \Delta, \Gamma$, and $M$, which satisfies the following property: if a curve $C^{\prime}$ in $X$ and an $\mathbb{R}$-divisor $B_{0} \in \mathcal{L}$ satisfy $\left\|B_{0}-\Delta\right\|<\delta,\left(K_{X}+\Gamma+B_{0}\right) \cdot C^{\prime} \leq 0$, and $-\left(K_{X}+\Gamma+B\right) \cdot C^{\prime} \leq M$ for all $B \in \mathcal{L}$, then $\left(K_{X}+\Gamma+\Delta\right) \cdot C^{\prime} \leq 0$.

(3) Let $\left\{C_{t}\right\}_{t \in T}$ be a set of curves such that $-\left(K_{X}+\Gamma+B\right) \cdot C_{t} \leq M$ for all $B \in \mathcal{L}$. Then, the set

$$
\mathcal{N}_{T}(\Gamma):=\left\{B \in \mathcal{L} \mid\left(K_{X}+\Gamma+B\right) \cdot C_{t} \geq 0 \text { for any } t \in T\right\}
$$

is a rational polytope.

Proof. Note that, for every $B \in \mathcal{L}$, we obtain the irreducible decomposition

$$
B=\sum_{i=1}^{s} l_{i} \Lambda_{i}
$$

for some real numbers $l_{i}$ with $0 \leq l_{i} \leq \lambda$.

(1) We can write $\Delta:=\sum l_{i} \Lambda_{i}$ as above. Then we have

$$
\left(K_{X}+\Gamma+\Delta\right) \cdot C=\sum l_{i}\left(K_{X}+\Gamma+\Lambda_{i}\right) \cdot C
$$

Suppose that $\left(K_{X}+\Gamma+\Delta\right) \cdot C<1$. Then we have

$$
\begin{aligned}
l_{i}\left(K_{X}+\Gamma+\Lambda_{i}\right) \cdot C & <1-\sum_{j \neq i} l_{j}\left(K_{X}+\Gamma+\Lambda_{j}\right) \cdot C \\
& \leq 1+\sum_{j \neq i} l_{j} M \\
& \leq 1+(s-1) \lambda M .
\end{aligned}
$$

Thus, if $l_{i} \neq 0$, then we obtain

$$
-M \leq\left(K_{X}+\Gamma+\Lambda_{i}\right) \cdot C<\frac{1}{l_{i}}(1+(s-1) \lambda M) .
$$

Since $X$ is $\mathbb{Q}$-factorial, the $\mathbb{Q}$-divisor $K_{X}+\Gamma+\Lambda_{i}$ is $\mathbb{Q}$-Cartier. This means that there are only finitely many possibilities for the number $\left(K_{X}+\Gamma+\right.$ $\left.\Lambda_{i}\right) \cdot C$.

Thus, if $\left(K_{X}+\Gamma+\Delta\right) \cdot C<1$, then there are only finitely many possibilities for the number $\left(K_{X}+\Gamma+\Delta\right) \cdot C=\sum l_{i}\left(K_{X}+\Gamma+\Lambda_{i}\right) \cdot C$. Therefore, we can find the desired number $\epsilon$. 
(2) Suppose that the statement is not true. Then, for an arbitrary positive real number $\delta$, there exist a curve $C^{\prime}$ and an $\mathbb{R}$-divisor $B_{0} \in \mathcal{L}$ which satisfy $\left\|B_{0}-\Delta\right\|<\delta,\left(K_{X}+\Gamma+B_{0}\right) \cdot C^{\prime} \leq 0,-\left(K_{X}+\Gamma+B\right) \cdot C^{\prime} \leq M$ for all $B \in \mathcal{L}$ and $\left(K_{X}+\Gamma+\Delta\right) \cdot C^{\prime}>0$. Set $\delta:=1 / m$ for any $m \in \mathbb{Z}_{>0}$. Then we obtain an infinite sequence of curves $C_{m}$ and $B_{m} \in \mathcal{L}$ which satisfy

$$
\begin{aligned}
& \left(K_{X}+\Gamma+B_{m}\right) \cdot C_{m} \leq 0, \\
& -\left(K_{X}+\Gamma+B\right) \cdot C_{m} \leq M \quad \text { for all } B \in \mathcal{L}, \quad \text { and } \\
& \quad\left(K_{X}+\Gamma+\Delta\right) \cdot C_{m}>0,
\end{aligned}
$$

and $\left\|B_{m}-\Delta\right\|$ converges to 0 . Let $\Delta=\sum l_{i} \Lambda_{i}$, and let $B_{m}=\sum l_{i, m} \Lambda_{i}$ as above. Then we see that $l_{i}=\lim l_{i, m}$. Here, for each $j$, the set $\left\{\left(K_{X}+\Gamma+\right.\right.$ $\left.\left.\Lambda_{j}\right) \cdot C_{m}\right\}_{m}$ has a lower bound $-M$.

We show that, if $l_{j} \neq 0$, then the set $\left\{\left(K_{X}+\Gamma+\Lambda_{j}\right) \cdot C_{m}\right\}_{m}$ has an upper bound. Since $0<l_{j}=\lim l_{j, m}$, we may assume that $l_{j, m}>0$ for all $m$ by replacing the sequence with a suitable subsequence. By the inequality

$$
0 \geq\left(K_{X}+\Gamma+B_{m}\right) \cdot C_{m}=\sum l_{i, m}\left(K_{X}+\Gamma+\Lambda_{i}\right) \cdot C_{m}
$$

we have

$$
\begin{aligned}
\left(K_{X}+\Gamma+\Lambda_{j}\right) \cdot C_{m} & \leq \frac{1}{l_{j, m}}\left(-\sum_{i \neq j} l_{i, m}\left(K_{X}+\Gamma+\Lambda_{i}\right) \cdot C_{m}\right) \\
& \leq \frac{1}{l_{j, m}}\left(\sum_{i \neq j} l_{i, m} M\right) \\
& \leq \frac{1}{l_{j, m}}(s-1) \lambda M .
\end{aligned}
$$

Since the set $\left\{1 / l_{j, m}\right\}_{m}$ has an upper bound, the set $\left\{\left(K_{X}+\Gamma+\Lambda_{j}\right) \cdot C_{m}\right\}_{m}$ also has an upper bound. This is what we want to show.

Then, for $m \gg 0$, we have

$$
\begin{aligned}
( & \left.K_{X}+\Gamma+B_{m}\right) \cdot C_{m} \\
& =\left(K_{X}+\Gamma+\Delta\right) \cdot C_{m}+\sum\left(l_{i, m}-l_{i}\right)\left(K_{X}+\Gamma+\Lambda_{i}\right) \cdot C_{m} \\
& >\epsilon+\sum\left(l_{i, m}-l_{i}\right)\left(K_{X}+\Gamma+\Lambda_{i}\right) \cdot C_{m} \\
& =\epsilon+\sum_{l_{i} \neq 0}\left(l_{i, m}-l_{i}\right)\left(K_{X}+\Gamma+\Lambda_{i}\right) \cdot C_{m}+\sum_{l_{i}=0} l_{i, m}\left(K_{X}+\Gamma+\Lambda_{i}\right) \cdot C_{m}
\end{aligned}
$$




$$
\begin{aligned}
& \geq \epsilon+\sum_{l_{i} \neq 0}\left(l_{i, m}-l_{i}\right)\left(K_{X}+\Gamma+\Lambda_{i}\right) \cdot C_{m}+\sum_{l_{i}=0} l_{i, m}(-M) \\
& >0 .
\end{aligned}
$$

The first inequality follows from (1). The third inequality follows when $m \gg 0$. Note that, if $l_{i} \neq 0$, then the set $\left\{\left(K_{X}+\Gamma+\Lambda_{i}\right) \cdot C_{m}\right\}_{i}$ is bounded from both sides. This is a contradiction.

(3) We show the assertion by the induction on $\operatorname{dim} \mathcal{L}$. If $\operatorname{dim} \mathcal{L}=0$, then there is nothing to show. Thus, we assume that $\operatorname{dim} \mathcal{L}>0$. We may assume that for each $t \in T$ there exists $B \in \mathcal{L}$ with $\left(K_{X}+\Gamma+B\right) \cdot C_{t}<0$.

We see that $\mathcal{N}_{T}(\Gamma)$ is a compact set. Then, by (2) and by the compactness of $\mathcal{N}_{T}(\Gamma)$, there exist $\mathbb{R}$-divisors $\Delta_{1}, \ldots, \Delta_{n} \in \mathcal{N}_{T}(\Gamma)$ and positive real numbers $\delta_{1}>0, \ldots, \delta_{n}>0$ such that $\mathcal{N}_{T}(\Gamma)$ is covered by $\mathcal{B}_{i}:=\{B \in \mathcal{L} \mid$ $\left.\left\|B-\Delta_{i}\right\|<\delta_{i}\right\}$ and such that if $B \in \mathcal{B}_{i}$ with $\left(K_{X}+\Gamma+B\right) \cdot C_{t}<0$ for some $t \in T$, then $\left(K_{X}+\Gamma+\Delta_{i}\right) \cdot C_{t}=0$. Set

$$
T_{i}:=\left\{t \in T \mid\left(K_{X}+\Gamma+B\right) \cdot C_{t}<0 \text { for some } B \in \mathcal{B}_{i}\right\}
$$

Then, for every $t \in T_{i}$, we have $\left(K_{X}+\Gamma+\Delta_{i}\right) \cdot C_{t}=0$. In particular, $\Delta_{i}$ is a $\mathbb{Q}$-divisor.

Here, we prove that

$$
\mathcal{N}_{T}(\Gamma)=\bigcap \mathcal{N}_{T_{i}}(\Gamma)
$$

The inclusion $\mathcal{N}_{T}(\Gamma) \subset \bigcap \mathcal{N}_{T_{i}}(\Gamma)$ is obvious. Thus, we want to prove that $\mathcal{N}_{T}(\Gamma) \supset \bigcap \mathcal{N}_{T_{i}}(\Gamma)$. Let $B \notin \mathcal{N}_{T}(\Gamma)$. Since $\mathcal{N}_{T}(\Gamma)$ is compact, we can find an element $B^{\prime} \in \mathcal{N}_{T}(\Gamma)$ with

$$
\left\|B^{\prime}-B\right\|=\min \left\{\left\|B^{*}-B\right\| \mid B^{*} \in \mathcal{N}_{T}(\Gamma)\right\} .
$$

Here we have $B^{\prime} \in \mathcal{B}_{i}$ for some $i$. Since $\mathcal{B}_{i} \cap \overline{B B^{\prime}}$ is an open subset of $\overline{B B^{\prime}}$ where $\overline{B B^{\prime}}$ is the line segment, we have an element $B^{\prime \prime}$ such that $B^{\prime \prime} \in$ $\mathcal{B}_{i} \cap \overline{B B^{\prime}}, B^{\prime \prime} \neq B$, and $B^{\prime \prime} \neq B^{\prime}$. This means that there is a real number $\beta$ with $0<\beta<1$ such that

$$
\beta B+(1-\beta) B^{\prime}=B^{\prime \prime}
$$

We obtain

$$
\beta\left(K_{X}+\Gamma+B\right)+(1-\beta)\left(K_{X}+\Gamma+B^{\prime}\right)=K_{X}+\Gamma+B^{\prime \prime} .
$$


Moreover, we see that $B^{\prime \prime} \notin \mathcal{N}_{T}(\Gamma)$. Here, since $B^{\prime \prime} \in \mathcal{B}_{i} \backslash \mathcal{N}_{T}(\Gamma)$, we have $\left(K_{X}+\Gamma+B^{\prime \prime}\right) \cdot C_{t}<0$ for some $t \in T_{i}$. Thus, we obtain the inequality

$$
\begin{aligned}
\beta\left(K_{X}+\Gamma+B\right) \cdot C_{t} & =\left(K_{X}+\Gamma+B^{\prime \prime}\right) \cdot C_{t}-(1-\beta)\left(K_{X}+\Gamma+B^{\prime}\right) \cdot C_{t} \\
& <-(1-\beta)\left(K_{X}+\Gamma+B^{\prime}\right) \cdot C_{t} \\
& \leq 0 .
\end{aligned}
$$

Therefore, we have $\left(K_{X}+\Gamma+B\right) \cdot C_{t}<0$. This means that $B \notin \mathcal{N}_{T_{i}}(\Gamma)$.

Therefore, it is enough to prove that each $\mathcal{N}_{T_{i}}(\Gamma)$ is a rational polytope. By replacing $T$ with $T_{i}$, we may assume that there exists a $\mathbb{Q}$-divisor $\Delta_{0} \in$ $\mathcal{N}_{T}(\Gamma)$ such that $\left(K_{X}+\Gamma+\Delta_{0}\right) \cdot C_{t}=0$ for every $t \in T$. Let $\mathcal{L}^{1}, \ldots, \mathcal{L}^{u}$ be the proper faces of $\mathcal{L}$ with codimension 1 . Note that, for every $1 \leq u^{\prime} \leq u$, there exists a positive integer $i^{\prime}$ such that

$$
\mathcal{L}^{u^{\prime}}=\left\{B \in \sum_{i \neq i^{\prime}} \mathbb{R} \Lambda_{i} \mid 0 \leq B \leq \lambda \sum_{i \neq i^{\prime}} \Lambda_{i}\right\}
$$

or such that

$$
\mathcal{L}^{u^{\prime}}=\lambda \Lambda_{i^{\prime}}+\left\{B \in \sum_{i \neq i^{\prime}} \mathbb{R} \Lambda_{i} \mid 0 \leq B \leq \lambda \sum_{i \neq i^{\prime}} \Lambda_{i}\right\}
$$

Let us prove that each $\mathcal{N}_{T}^{u^{\prime}}(\Gamma):=\mathcal{N}_{T}(\Gamma) \cap \mathcal{L}^{u^{\prime}}$ is a rational polytope. If $\mathcal{L}^{u^{\prime}}$ satisfies equation (I), then we see that

$$
\mathcal{N}_{T}^{u^{\prime}}(\Gamma)=\left\{B \in \mathcal{L}^{u^{\prime}} \mid\left(K_{X}+\Gamma+B\right) \cdot C_{t} \geq 0 \text { for any } t \in T\right\} .
$$

Hence, $\mathcal{N}_{T}^{u^{\prime}}(\Gamma)$ is a rational polytope by the induction hypothesis. Thus, assume that $\mathcal{L}^{u^{\prime}}$ satisfies equation (II). Set $\mathcal{L}_{0}^{u^{\prime}}:=\left\{B \in \sum_{i \neq i^{\prime}} \mathbb{R} \Lambda_{i} \mid 0 \leq B \leq\right.$ $\left.\lambda \sum_{i \neq i^{\prime}} \Lambda_{i}\right\}$. Equation (II) implies that

$$
\mathcal{L}^{u^{\prime}}=\lambda \Lambda_{i^{\prime}}+\mathcal{L}_{0}^{u^{\prime}}
$$

Then we see that

$$
\begin{aligned}
\mathcal{N}_{T}^{u^{\prime}}(\Gamma) & =\left\{B \in \mathcal{L}^{u^{\prime}} \mid\left(K_{X}+\Gamma+B\right) \cdot C_{t} \geq 0 \text { for any } t \in T\right\} \\
& =\lambda \Lambda_{i^{\prime}}+\left\{B_{0} \in \mathcal{L}_{0}^{u^{\prime}} \mid\left(K_{X}+\Gamma+\lambda \Lambda_{i^{\prime}}+B_{0}\right) \cdot C_{t} \geq 0 \text { for any } t \in T\right\} .
\end{aligned}
$$

For all $B_{0} \in \mathcal{L}_{0}^{u^{\prime}}$, we have the inequality

$$
-\left(K_{X}+\Gamma+\lambda \Lambda_{i^{\prime}}+B_{0}\right) \cdot C_{t} \leq M
$$


Thus, the set

$$
\begin{aligned}
& \mathcal{N}_{T}\left(\mathcal{L}_{0}^{u^{\prime}}, \Gamma+\lambda \Lambda_{i^{\prime}}\right) \\
& \quad:=\left\{B_{0} \in \mathcal{L}_{0}^{u^{\prime}} \mid\left(K_{X}+\Gamma+\lambda \Lambda_{i^{\prime}}+B_{0}\right) \cdot C_{t} \geq 0 \text { for any } t \in T\right\}
\end{aligned}
$$

is a rational polytope by the induction hypothesis. Therefore, $\mathcal{N}_{T}^{u^{\prime}}(\Gamma)$ is also a rational polytope, and this is what we want to show.

Here, take an arbitrary element $B \in \mathcal{N}_{T}(\Gamma)$ with $B \neq \Delta_{0}$. Then we can find $B^{\prime} \in \mathcal{L}^{u^{\prime}}$ for some $1 \leq u^{\prime} \leq u$ such that $B$ is on the line segment defined by $\Delta_{0}$ and $B^{\prime}$. Since $\left(K_{X}+\Gamma+\Delta_{0}\right) \cdot C_{t}=0$ for all $t \in T$, we have $B^{\prime} \in$ $\mathcal{N}_{T}^{u^{\prime}}(\Gamma)$. Thus, we see that $\mathcal{N}_{T}(\Gamma)$ is the convex hull of $\Delta_{0}$ and all the $\mathcal{N}_{T}^{u^{\prime}}(\Gamma)$. Hence, $\mathcal{N}_{T}(\Gamma)$ is a rational polytope.

Corollary 3.40. Let $\left\{R_{t}\right\}_{t \in T}$ be a family of extremal rays of $\overline{N E}(X)$ spanned by curves. Then the set

$$
\mathcal{N}_{T}:=\left\{B \in \mathcal{L} \mid\left(K_{X}+B\right) \cdot R_{t} \geq 0 \text { for any } t \in T\right\}
$$

is a rational polytope.

Proof. By Lemma 3.37, for every $t \in T$ there exists a curve $C_{t}$ such that $R_{t}=\mathbb{R}_{\geq 0}\left[C_{t}\right]$ and $-\left(K_{X}+B\right) \cdot C_{t} \leq M(X, \mathcal{L})$ for all $B \in \mathcal{L}$. Let $\Gamma:=0$, and let $M:=M(X, \mathcal{L})$. Then we can apply Proposition 3.39. Therefore, the set $\mathcal{N}_{T}=\mathcal{N}_{T}(0)$ is a rational polytope.

Now, we prove the abundance theorem with $\mathbb{R}$-coefficients.

TheOREM 3.41. Let $X$ be a projective normal $\mathbb{Q}$-factorial surface over $k$, and let $\Delta$ be an $\mathbb{R}$-boundary. If $k$ is not the algebraic closure of a finite field and $K_{X}+\Delta$ is nef, then $K_{X}+\Delta$ is semiample.

Proof. Let $\left\{R_{t}\right\}_{t \in T}$ be the set of all the extremal rays of $\overline{N E}(X)$ spanned by curves. Then

$$
\mathcal{N}_{T}:=\left\{B \in \mathcal{L} \mid\left(K_{X}+B\right) \cdot R_{t} \geq 0 \text { for every } t \in T\right\}
$$

is a rational polytope by Corollary 3.40. Moreover, by Theorem 3.13, we see that

$$
\begin{aligned}
\mathcal{N}_{T} & =\left\{B \in \mathcal{L} \mid\left(K_{X}+B\right) \cdot R_{t} \geq 0 \text { for every } t \in T\right\} \\
& =\left\{B \in \mathcal{L} \mid K_{X}+B \text { is nef }\right\}
\end{aligned}
$$


Since $\Delta \in \mathcal{N}_{T}$, we can find $\mathbb{Q}$-divisors $\Delta_{1}, \ldots, \Delta_{l}$ such that $\Delta_{i} \in \mathcal{N}_{T}$ for all $i$ and such that $\sum r_{i} \Delta_{i}=\Delta$, where positive real numbers $r_{i}$ satisfy $\sum r_{i}=1$. Thus, we have

$$
K_{X}+\Delta=\sum r_{i}\left(K_{X}+\Delta_{i}\right),
$$

and $K_{X}+\Delta_{i}$ is nef. By Theorem 3.33, $K_{X}+\Delta_{i}$ is semiample.

\section{$\S 4$. Normal surfaces over $\overline{\mathbb{F}}_{p}$}

\subsection{Contraction problem}

In this section, let $k$ be an arbitrary algebraically closed field, and let char $k=p \geq 0$. As the introduction of this part, we consider the following question.

QUESTION 4.1 (Contraction problem). Let $X$ be a smooth projective surface over $k$, and let $C$ be a curve in $X$. If $C^{2}<0$, then is $C$ contractible? That is, does there exist a birational morphism $f: X \rightarrow Y$ to an algebraic surface $Y$ such that $f\left(C^{\prime}\right)$ is one point if and only if $C^{\prime}=C$ for every curve $C^{\prime} ?$

ANSwer 4.2. If $k \neq \overline{\mathbb{F}}_{p}$, then the answer to Question 4.1 is no in general.

We only recall the method of its construction. For more details, see $[\mathrm{H}$, Example 5.7.3].

Construction. If we obtain an elliptic curve $C_{0}$ in $\mathbb{P}^{2}$ with rank at least 10 , then we can construct a counterexample as follows. There are 10 points in $C_{0}$ which are linearly independent. Blow up $\mathbb{P}^{2}$ at these 10 points. The proper transform $C$ of $C_{0}$ is not contractible.

By Fact 2.3, if $k \neq \overline{\mathbb{F}}_{p}$, then we can use this construction. On the other hand, if $k=\overline{\mathbb{F}}_{p}$, then we have the opposite answer.

Answer 4.3 ([A, Theorem 2.9]). If $k=\overline{\mathbb{F}}_{p}$, then the answer to Question 4.1 is yes.

To see this answer and its mechanism of this proof, we divide the verification into small pieces and prove the more general following result.

Proposition 4.4. Let $X$ be a projective normal $\mathbb{Q}$-factorial surface over $k$, and let $C$ be a curve in $X$.

(1) If $C^{2}<0$, then there exists a nef and big divisor $G$ such that $G \cdot C^{\prime}=0$ if and only if $C^{\prime}=C$ for any curve $C^{\prime}$ in $X$. 
(2) If the restriction $\left.G\right|_{C}$ of the divisor $G$ in (1) is a torsion, and if char $k=$ $p>0$, then $G$ is semiample.

(3) If $k=\overline{\mathbb{F}}_{p}$, then $\left.G\right|_{C}$ is torsion.

Proof. (1) Let $H$ be an ample divisor on $X$. We define a $\mathbb{Q}$-divisor $G$ and $q \in \mathbb{Q}_{>0}$ by $G=H+q C$ and $G \cdot C=0$. It is easy to check that $G$ satisfies the above conditions.

(2) Since $p>0$, we can use Keel's result (Theorem 2.2). Therefore, the semiampleness of $G$ is equivalent to the semiampleness of $\left.G\right|_{C}$. But $\left.G\right|_{C}$ is a torsion by the assumption. Thus, $G$ is semiample.

(3) This is an immediate consequence of Corollary 2.4.

For results related to this section, see [A] and [Bă2].

\section{2. $\mathbb{Q}$-factoriality}

In this section, we prove the following two theorems.

TheOREM 4.5. If $X$ is a normal surface over $\overline{\mathbb{F}}_{p}$, then $X$ is $\mathbb{Q}$-factorial.

TheOREM 4.6. Let $f: X \rightarrow Y$ be a proper birational morphism between normal surfaces over $\overline{\mathbb{F}}_{p}$. Then $f$ factors into contractions of one curve. More precisely, there exist proper birational morphisms such that each $g_{i}$ : $X_{i} \rightarrow X_{i+1}$ is a proper birational morphism between normal surfaces such that $\operatorname{Ex}\left(g_{i}\right)$ is an irreducible curve.

The following lemma plays a key role in this section.

Lemma 4.7. Let $f: X \rightarrow Y$ be a proper birational morphism over $\overline{\mathbb{F}}_{p}$ from a normal $\mathbb{Q}$-factorial surface $X$ to a normal surface $Y$. Let $\operatorname{Ex}(f)=$ $C_{1} \cup \cdots \cup C_{r}$.

(1) There exists a proper birational morphism $g: X \rightarrow Z$ to a normal surface $Z$ such that $\operatorname{Ex}(g)=C_{1}$.

(2) The morphism $f$ factors through $Z$.

(3) The surface $Z$ is $\mathbb{Q}$-factorial.

Proof. (1) If $X$ and $Y$ are proper, then the assertion follows from Proposition 4.4. Note that proper $\mathbb{Q}$-factorial surfaces are projective (see [F2, Lemma 2.2]). In the general case, take the Nagata compactification. Note that normality and $\mathbb{Q}$-factoriality may break up by compactification. However, by taking the normalization and the resolution of the locus of $\bar{X} \backslash X$, we may make these assumptions.

(2) This is obvious. 
(3) The assertion immediately follows from Propositions 3.25, 3.26, and 4.4 .

Corollary 4.8. Let $f: X \rightarrow Y$ be a proper birational morphism over $\overline{\mathbb{F}}_{p}$ from a normal $\mathbb{Q}$-factorial surface $X$ to a normal surface $Y$. Then $Y$ is $\mathbb{Q}$-factorial.

Proof. By using Lemma 4.7 repeatedly, $f$ is factored into contractions of one curve, and $\mathbb{Q}$-factoriality of $X$ descends to $Y$.

By the same argument, Theorem 4.6 follows from Theorem 4.5. Thus, we prove only Theorem 4.5 .

Proof of Theorem 4.5. Let $f: X^{\prime} \rightarrow X$ be the resolution of singularities. Of course, $X^{\prime}$ is $\mathbb{Q}$-factorial. Therefore, $X$ is also $\mathbb{Q}$-factorial by Corollary 4.8 .

REMARK 4.9. Theorem 4.5 follows from [Bă1, Corollary 14.22] and [Ma, (24.E)].

\subsection{Theorems in Section 3}

In this section, we establish the theorems, which we discussed in Section 3, over $\overline{\mathbb{F}}_{p}$ under much weaker assumptions.

TheOREM 4.10 (Contraction theorem). Let $X$ be a projective normal surface over $\overline{\mathbb{F}}_{p}$, and let $\Delta$ be an effective $\mathbb{R}$-divisor. Let $R=\mathbb{R}_{\geq 0}[C]$ be a $\left(K_{X}+\Delta\right)$-negative extremal ray. Then there exists a surjective morphism $\phi_{R}: X \rightarrow Y$ to a projective variety $Y$ with the following properties:

(1) let $C^{\prime}$ be a curve on $X$, and then $\phi_{R}\left(C^{\prime}\right)$ is one point if and only if $\left[C^{\prime}\right] \in R$

(2) $\left(\phi_{R}\right)_{*}\left(\mathcal{O}_{X}\right)=\mathcal{O}_{Y}$;

(3) if $L$ is an invertible sheaf with $L \cdot C=0$, then $n L=\left(\phi_{R}\right)^{*} L_{Y}$ for some invertible sheaf $L_{Y}$ on $Y$ and for some positive integer $n$;

(4) $\rho(Y)=\rho(X)-1$.

Proof. If $C^{2} \geq 0$, then we have

$$
K_{X} \cdot C \leq\left(K_{X}+\Delta\right) \cdot C<0
$$

Then we can apply Theorem 3.21. Thus, we may assume that $C^{2}<0$. But this curve is contractible, and the proofs of the remaining properties are the same as those of Theorem 3.21. 
The following theorem is a known result (see [Bă1, Corollary 14.29]). We give a minimal model-theoretic proof.

THEOREM 4.11 (Finite generation theorem). Let $X$ be a projective normal surface over $\overline{\mathbb{F}}_{p}$, and let $D$ be a $\mathbb{Q}$-divisor. Then $R(X, D)=\bigoplus_{m \geq 0} H^{0}(X$, $\llcorner m D\lrcorner)$ is a finitely generated $\overline{\mathbb{F}}_{p}$-algebra.

Proof. We may assume that $\kappa(X, D) \geq 1$. Then, in particular, $D$ is effective. If there is a curve with $D \cdot C<0$, then $C^{2}<0$ and $C$ is contractible. Let $f: X \rightarrow Y$ be the contraction of $C$. Note that we obtain $D=f^{*} f_{*} D+q C$, for a positive rational number $q$. Therefore, we may assume that $D$ is nef. If $\kappa(X, D)=1$, then $D$ is semiample by Proposition 3.23. If $\kappa(X, D)=2$, then $D$ is semiample by the following proposition.

Proposition 4.12. Let $X$ be a projective normal surface over $\overline{\mathbb{F}}_{p}$. If $D$ is a nef and big $\mathbb{Q}$-divisor, then $D$ is semiample.

Proof. If there is a curve $C$ such that $D \cdot C=0$, then $C^{2}<0$ and $C$ is contractible. Let $f: X \rightarrow Y$ be its contraction, and let $f^{*} D_{Y}=D$. It is sufficient to prove that $D_{Y}$ is semiample. Repeating the same procedure, we see that $D$ is a pullback of an ample divisor.

Theorem 4.13 (Nonvanishing theorem). Let $X$ be a projective normal surface over $\overline{\mathbb{F}}_{p}$, and let $\Delta$ be an effective $\mathbb{Q}$-divisor. If $K_{X}+\Delta$ is nef, then $\kappa\left(X, K_{X}+\Delta\right) \geq 0$.

The proof of this theorem depends heavily on the argument in [M, Theorem 2].

Proof of Theorem 4.13. We may assume that $X$ is smooth by replacing it with its minimal resolution.

STEP 1. If $\kappa\left(X, K_{X}\right) \geq 0$, then $\kappa\left(X, K_{X}+\Delta\right) \geq \kappa\left(X, K_{X}\right) \geq 0$. Thus, we may assume that $\kappa\left(X, K_{X}\right)=-\infty$.

STEP 2. In this step, we show that we may assume that $K_{X}+\Delta$ is not numerically trivial and that $h^{2}\left(X, m\left(K_{X}+\Delta\right)\right)=0$ for $m \gg 0$.

If $K_{X}+\Delta$ is numerically trivial, then $K_{X}+\Delta$ is a torsion by Fact 2.3. Thus, we obtain $n\left(K_{X}+\Delta\right) \sim 0$ for some integer $n$ and $\kappa\left(X, K_{X}+\Delta\right)=0$. Therefore, we may assume that $K_{X}+\Delta$ is not numerically trivial. Then we obtain $h^{2}\left(X, m\left(K_{X}+\Delta\right)\right)=h^{0}\left(X, K_{X}-m\left(K_{X}+\Delta\right)\right)=0$ for $m \gg 0$. (We have $\left(K_{X}+\Delta\right) \cdot C>0$ for some curve. Then there exist an ample divisor $A$ and an effective divisor $E$ such that $A=C+E$. By the nefness of $K_{X}+\Delta$, 
we obtain $\left(K_{X}+\Delta\right) \cdot A>0$. Then since $\left(K_{X}-m\left(K_{X}+\Delta\right)\right) \cdot A<0$ for sufficiently large integer $m$, we obtain $h^{0}\left(X, K_{X}-m\left(K_{X}+\Delta\right)\right)=0$.)

STEP 3. In this step we show that we may assume that $\left(K_{X}+\Delta\right)^{2}=0$.

Suppose the contrary; that is, suppose that $\left(K_{X}+\Delta\right)^{2}>0$. Then $K_{X}+\Delta$ is nef and big. Then we obtain $h^{0}\left(X, m\left(K_{X}+\Delta\right)\right)>0$ for some positive integer $m$, and $\kappa\left(X, K_{X}+\Delta\right) \geq 0$.

We consider the two cases: $X$ is rational or irrational.

SteP 4. In this step, we prove the assertion when $X$ is rational.

Now $\chi\left(\mathcal{O}_{X}\right)=1$ because $X$ is rational. Then, the Riemann-Roch theorem shows that

$$
\begin{aligned}
& h^{0}\left(X, m\left(K_{X}+\Delta\right)\right) \\
& \quad=h^{1}\left(X, m\left(K_{X}+\Delta\right)\right)+1+\frac{1}{2} m\left(K_{X}+\Delta\right) \cdot\left(m\left(K_{X}+\Delta\right)-K_{X}\right),
\end{aligned}
$$

where $m \gg 0$. The right-hand side is positive because

$$
\begin{aligned}
& m\left(K_{X}+\Delta\right) \cdot\left(m\left(K_{X}+\Delta\right)-K_{X}\right) \\
& \quad=m\left(K_{X}+\Delta\right) \cdot\left((m-1)\left(K_{X}+\Delta\right)+\Delta\right) \geq 0
\end{aligned}
$$

by the nefness of $K_{X}+\Delta$. This is what we want to show.

Thus, we may assume that $X$ is an irrational ruled surface. We divide the proof into three cases: $\left(K_{X}+\Delta\right) \cdot K_{X}<0,\left(K_{X}+\Delta\right) \cdot K_{X}>0$, and $\left(K_{X}+\Delta\right) \cdot K_{X}=0$.

SteP 5. We assume that $X$ is irrational and that $\left(K_{X}+\Delta\right) \cdot K_{X}<0$.

By Steps 2 and 3 and the Riemann-Roch theorem, $h^{0}\left(X, m\left(K_{X}+\Delta\right)\right)>0$ for some large integer $m$. This is what we want to show.

STEP 6. We assume that $X$ is irrational and that $\left(K_{X}+\Delta\right) \cdot K_{X}>0$.

Since $\left(K_{X}+\Delta\right)^{2}=0$ and $\left(K_{X}+\Delta\right) \cdot K_{X}>0$, we obtain $\left(K_{X}+\Delta\right) \cdot \Delta<0$. This contradicts the nefness of $K_{X}+\Delta$.

STEP 7. We assume that $X$ is irrational and that $\left(K_{X}+\Delta\right) \cdot K_{X}=0$.

We assume that $\kappa\left(X, K_{X}+\Delta\right)=-\infty$ and derive a contradiction. By $\left(K_{X}+\Delta\right) \cdot K_{X}=0$ and $\left(K_{X}+\Delta\right)^{2}=0$, we obtain $\left(K_{X}+\Delta\right) \cdot \Delta=0$. Let $C$ be an arbitrary prime component of $\Delta$. Since $\Delta \neq 0$, we can take such a curve. (Indeed, if $\Delta=0$, then $K_{X}$ is nef. This contradicts that $X$ is 
a ruled surface.) By $\left(K_{X}+\Delta\right) \cdot \Delta=0$ and the nefness of $K_{X}+\Delta$, we have $\left(K_{X}+\Delta\right) \cdot C=0$. By Fact 2.3 , we obtain $\left.n_{1}\left(K_{X}+\Delta\right)\right|_{C} \sim 0$ for some $n_{1} \in \mathbb{Z}_{>0}$. Then we get the exact sequence

$$
0 \rightarrow \mathcal{O}_{X}\left(n_{1} n_{2}\left(K_{X}+\Delta\right)-C\right) \rightarrow \mathcal{O}_{X}\left(n_{1} n_{2}\left(K_{X}+\Delta\right)\right) \rightarrow \mathcal{O}_{C} \rightarrow 0
$$

for every $n_{2} \in \mathbb{Z}_{>0}$. Here we want to prove that, for every $n_{2} \gg 0$,

$$
h^{2}\left(X, n_{1} n_{2}\left(K_{X}+\Delta\right)-C\right)=0 .
$$

By Serre duality, we obtain $h^{2}\left(X, n_{1} n_{2}\left(K_{X}+\Delta\right)-C\right)=h^{0}\left(X, K_{X}+C-\right.$ $\left.n_{1} n_{2}\left(K_{X}+\Delta\right)\right)$. This is 0 , by the same argument as in Step 2 .

Fix $n_{2} \gg 0$, and let $n:=n_{1} n_{2}$. By $h^{2}\left(X, n\left(K_{X}+\Delta\right)-C\right)=0$, we have a surjection $H^{1}\left(X, n\left(K_{X}+\Delta\right)\right) \rightarrow H^{1}\left(C, \mathcal{O}_{C}\right)$. This means that

$$
h^{1}\left(X, n\left(K_{X}+\Delta\right)\right) \geq h^{1}\left(C, \mathcal{O}_{C}\right)
$$

On the other hand, by $h^{0}\left(X, n\left(K_{X}+\Delta\right)\right)=h^{2}\left(X, n\left(K_{X}+\Delta\right)\right)=0$ and the Riemann-Roch theorem,

$$
\begin{aligned}
-h^{1}\left(X, n\left(K_{X}+\Delta\right)\right) & =\chi\left(\mathcal{O}_{X}\right)+\frac{1}{2} n\left(K_{X}+\Delta\right) \cdot\left\{n\left(K_{X}+\Delta\right)-K_{X}\right\} \\
& =\chi\left(\mathcal{O}_{X}\right)=1-h^{1}\left(B, \mathcal{O}_{B}\right),
\end{aligned}
$$

where $\pi: X \rightarrow B$ is the ruling. Hence, we have

$$
h^{1}\left(B, \mathcal{O}_{B}\right)-1=h^{1}\left(X, n\left(K_{X}+\Delta\right)\right) \geq h^{1}\left(C, \mathcal{O}_{C}\right)
$$

This shows that $C$ is in some fiber of $\pi$. In particular, for a smooth fiber $F$, we have $C \cdot F=0$. Recall that $C$ is an arbitrary prime component of $\Delta$; then we obtain $\Delta \cdot F=0$. Thus, we have

$$
0 \leq\left(K_{X}+\Delta\right) \cdot F=K_{X} \cdot F=-2
$$

This is a contradiction.

THEOREM 4.14 (Abundance theorem). Let $X$ be a projective normal surface over $\overline{\mathbb{F}}_{p}$, and let $\Delta$ be an effective $\mathbb{R}$-divisor. If $K_{X}+\Delta$ is nef, then $K_{X}+\Delta$ is semiample. 
Proof. By the same proof as Theorem 3.41, we may assume that $\Delta$ is a $\mathbb{Q}$-divisor. By Theorem 4.13, we have $\kappa\left(X, K_{X}+\Delta\right) \geq 0$. By Propositions 3.23 and 4.12 , we may assume that $\kappa\left(X, K_{X}+\Delta\right)=0$. Then we can apply the argument of Steps 1 and 2 in Theorem 3.34. By Proposition 3.32(1), we have $\kappa(S, Y)=1$ for indecomposable curves of canonical type $Y$ in $S$ over $\overline{\mathbb{F}}_{p}$. This contradicts $Z \neq 0$ and $\kappa(S, Z)=0$.

As an immediate corollary, we obtain the following base-point-free theorem.

THEOREM 4.15 (Base-point-free theorem). Let $X$ be a projective normal surface over $\overline{\mathbb{F}}_{p}$, and let $D$ be a nef divisor. If $\kappa\left(X, q D-K_{X}\right) \geq 0$ for some positive rational number $q$, then $D$ is semiample.

Proof. Take $q D-K_{X} \sim_{\mathbb{Q}} \Delta$. We obtain $q D \sim_{\mathbb{Q}} K_{X}+\Delta$ and can apply the abundance theorem.

\subsection{Examples}

In this section, let $k$ be an algebraically closed field of arbitrary characteristic. We want to see the difference between $k=\overline{\mathbb{F}}_{p}$ and $k \neq \overline{\mathbb{F}}_{p}$ by looking at some examples.

EXAmple 4.16 (See Theorems 3.21 and 4.10). If $k \neq \overline{\mathbb{F}}_{p}$, then there exist a smooth projective surface $X$ over $k$ and an elliptic curve $C$ in $X$ such that, for an arbitrary positive real number $\epsilon,\left(K_{X}+(1+\epsilon) C\right) \cdot C<0, C^{2}<0$, and $C$ is not contractible.

Construction. Consider Answer 4.2 and its construction. There exist a smooth projective surface $X$ and an elliptic curve $C$ in $X$ such that $C^{2}=-1$ and $C$ is not contractible. Moreover, we have

$$
\begin{aligned}
\left(K_{X}+(1+\epsilon) C\right) \cdot C & =\left(K_{X}+C\right) \cdot C+\epsilon C \cdot C \\
& =\epsilon C \cdot C<0 .
\end{aligned}
$$

This is what we want to show.

ExAMPlE 4.17 (See Theorems 3.30 and 4.13). If $k \neq \overline{\mathbb{F}}_{p}$, then there exist a smooth projective surface $X$ over $k$ and curves $C_{1}$ and $C_{2}$ in $X$ such that

$$
\begin{aligned}
K_{X}+(1+\epsilon) C_{1}+(1-\epsilon) C_{2} & \equiv 0 \quad \text { and } \\
\kappa\left(X, K_{X}+(1+\epsilon) C_{1}+(1-\epsilon) C_{2}\right) & =-\infty
\end{aligned}
$$

for an arbitrary positive rational number $\epsilon$. 
Construction. Let $P:=\mathbb{P}^{1}$, and let $E$ be an arbitrary elliptic curve. Set $X_{0}:=P \times E$. We construct $X$ by applying the elementary transform to the $\mathbb{P}^{1}$-bundle $X_{0}$ at two appropriate points. Let $e_{1}$ and $e_{2}$ be points in $E$ which are linearly independent. Fix two different points $p_{1}$ and $p_{2}$ in $P$, and set $S_{1}:=\left\{p_{1}\right\} \times E$ and $S_{2}:=\left\{p_{2}\right\} \times E$. Then we see that

$$
K_{X_{0}} \sim_{\mathbb{Q}}-(1+\epsilon) S_{1}-(1-\epsilon) S_{2}
$$

for an arbitrary rational number $\epsilon$. Let $x_{1}:=\left(p_{1}, e_{1}\right)$ and let $x_{2}:=\left(p_{2}, e_{2}\right)$. We take the elementary transform of $X_{0}$ at $x_{1}$ and $x_{2}$ and obtain $X$. (First, blow up at $x_{1}$. Then the proper transform of the fiber through $x_{1}$ is a $(-1)$ curve. Second, contract this $(-1)$-curve and get another $\mathbb{P}^{1}$-bundle. Repeat the same thing at $x_{2}$.) Let $C_{1}$ and $C_{2}$ be the proper transforms of $S_{1}$ and $S_{2}$, respectively, and let $F_{1}$ and $F_{2}$ be the fibers corresponding to $x_{1}$ and $x_{2}$, respectively. Then we see that

$$
K_{X} \sim_{\mathbb{Q}}-(1+\epsilon) C_{1}-(1-\epsilon) C_{2}-\epsilon F_{1}+\epsilon F_{2},
$$

which implies that

$$
K_{X}+(1+\epsilon) C_{1}+(1-\epsilon) C_{2} \sim_{\mathbb{Q}} \epsilon\left(-F_{1}+F_{2}\right)
$$

This divisor is numerically trivial. Here we want to show that $\kappa\left(X,-F_{1}+\right.$ $\left.F_{2}\right)=-\infty$, that is, that $-F_{1}+F_{2}$ is not a torsion. Consider the ruling $\pi: X \rightarrow E$ and one of its sections $\sigma: E \rightarrow X$. Then we have $F_{1}=\pi^{*} e_{1}$ and $F_{2}=\pi^{*} e_{2}$. Linear independence of $e_{1}$ and $e_{2}$ shows that

$$
\mathcal{O}_{X}\left(n\left(-F_{1}+F_{2}\right)\right) \not \mathcal{O}_{X}
$$

Indeed, if $\mathcal{O}_{X}\left(n\left(-F_{1}+F_{2}\right)\right) \simeq \mathcal{O}_{X}$, then we have $\pi^{*} \mathcal{O}_{E}\left(n\left(-e_{1}+e_{2}\right)\right) \simeq \mathcal{O}_{X}$ Then, we obtain

$$
\mathcal{O}_{E}\left(n\left(-e_{1}+e_{2}\right)\right) \simeq \sigma^{*} \pi^{*} \mathcal{O}_{E}\left(n\left(-e_{1}+e_{2}\right)\right) \simeq \sigma^{*} \mathcal{O}_{X} \simeq \mathcal{O}_{E}
$$

This is a contradiction.

EXAMPLE 4.18 (See Theorems 3.33 and 4.14). If $k \neq \overline{\mathbb{F}}_{p}$, then there exist a projective smooth surface $X$ over $k$ and an elliptic curve $C$ in $X$ such that, for an arbitrary positive rational number $\epsilon, K_{X}+(1+\epsilon) C$ is nef, $\kappa\left(X, K_{X}+(1+\epsilon) C\right) \geq 0$, and $K_{X}+(1+\epsilon) C$ is not semiample. 
Construction. Set $X_{0}:=\mathbb{P}^{2}$. Let $C_{0}$ be an arbitrary elliptic curve in $X_{0}$, and let $P_{1}, \ldots, P_{9}$ be points in $C_{0}$ which are linearly independent. Blow up at these nine points; then we obtain the surface $X$. Let $C$ be the proper transform of $C_{0}$. By $K_{X_{0}}=-C_{0}$, we have $K_{X}=-C$. Then

$$
K_{X}+(1+\epsilon) C=\epsilon C
$$

is nef by $C^{2}=0$. It is obvious that $\kappa\left(X, K_{X}+(1+\epsilon) C\right) \geq 0$. We prove that $K_{X}+(1+\epsilon) C$ is not semiample. It is sufficient to prove that $\kappa(X, C)=0$. Suppose the contrary; that is, suppose that $\kappa(X, C) \geq 1$. Then we obtain $n C \sim D$ for some nonzero effective divisor $D$ with $C \not \subset \operatorname{Supp} D$. Since $C$. $D=0, \operatorname{Supp}\left(\left.f_{*}(D)\right|_{C_{0}}\right)$ must be contained in $P_{1}, \ldots, P_{9}$. This means that $n_{1} P_{1}+\cdots+n_{9} P_{9}:=\left.\left.f_{*}(D)\right|_{C_{0}} \sim 3 n L\right|_{C_{0}}$. Here $L$ is a line in $X_{0}$. But this means that $n_{1} P_{1}+\cdots+n_{9} P_{9}=0$ in the group structure of $C_{0}$. This is a contradiction.

\section{$\S 5$. Log canonical surfaces}

\subsection{Log canonical singularities}

In this section, we describe the log canonical singularities in surfaces by using the contraction theorem (Theorem 3.21).

Definition 5.1. We say that a pair $(X, \Delta)$ is a $\log$ canonical surface if a normal surface $X$ and an $\mathbb{R}$-divisor $\Delta$ satisfy the following properties:

(1) $K_{X}+\Delta$ is $\mathbb{R}$-Cartier;

(2) for an arbitrary proper birational morphism $f: Y \rightarrow X$ and the divisor $\Delta_{Y}$ defined by

$$
K_{Y}+\Delta_{Y}=f^{*}\left(K_{X}+\Delta\right)
$$

the inequality $\Delta_{Y} \leq 1$ holds;

(3) $\Delta$ is effective.

First, we pay attention to only one singular point.

Definition 5.2. We say that $(X, \Delta)$ is a local situation of a log canonical surface if it satisfies the following properties.

(1) The pair $(X, \Delta)$ is a log canonical surface.

(2) There exists only one singular point $x \in X$.

(3) All prime components of $\Delta$ contain $x$. 
THEOREM 5.3. Let $(X, \Delta)$ be a local situation of a log canonical surface, and let $f: Y \rightarrow X$ be the minimal resolution of $X$. Then, there exists a sequence of proper birational morphisms

$$
f: Y=: Y_{0} \stackrel{f_{0}}{\rightarrow} Y_{1} \stackrel{f_{1}}{\rightarrow} \cdots \stackrel{f_{m-1}}{\rightarrow} Y_{m}=: Z \stackrel{g}{\rightarrow} X
$$

with the following properties.

(1) Each $Y_{i}$ is a normal $\mathbb{Q}$-factorial surface.

(2) Each $f_{i}$ is a proper birational morphism, and $E_{i}:=\operatorname{Ex}\left(f_{i}\right)$ is an irreducible curve.

(3) Each $E_{i}$ satisfies $\left(K_{Y_{i}}+E_{i}\right) \cdot E_{i}<0$.

(4) Either (a) or (b) holds:

(a) $g$ is an isomorphism;

(b) $\Delta=0$, and $E:=\operatorname{Ex}(g)$ is an irreducible curve such that $\left(K_{Y}+E\right)$. $E=0$.

Proof. We assume that we obtain

$$
f: Y=: Y_{0} \stackrel{f_{0}}{\rightarrow} Y_{1} \stackrel{f_{1}}{\rightarrow} \cdots \stackrel{f_{j-1}}{\rightarrow} Y_{j} \stackrel{G}{\rightarrow} X
$$

such that each $Y_{i}$ (as well as each $f_{i}$ ) satisfies (1), (2), and (3).

We prove that, if we can find a $G$-exceptional proper curve $E_{j}$ such that $\left(K_{Y_{j}}+E_{j}\right) \cdot E_{j}<0$, then we obtain a contraction of $E_{j}$

$$
f_{j}: Y_{j} \rightarrow Y_{j+1}
$$

to a $\mathbb{Q}$-factorial surface $Y_{j+1}$. If $X$ and $Y_{j}$ are proper, then we obtain the required morphism $f_{j}$ by Theorem 3.19 and Propositions 3.24, 3.25, and 3.26. Note that a proper $\mathbb{Q}$-factorial surface is projective (see $[\mathrm{F} 2$, Lemma 2.2]). For the general case, take compactifications as follows. Let $\bar{X}$ be a proper normal surface, and let $\bar{\Delta}$ be an $\mathbb{R}$-divisor on $\bar{X}$ such that $X \hookrightarrow \bar{X}$ is an open immersion, $(\bar{X}, \bar{\Delta})$ is a local situation of log canonical surface, and $\left.\bar{\Delta}\right|_{X}=\Delta$. We define $\overline{Y_{j}}$ by patching $Y_{j}$ and $\bar{X}$ along $Y_{j} \backslash \operatorname{Ex}(G) \simeq X \backslash\{x\}$. Then, $\overline{Y_{j}}$ is $\mathbb{Q}$-factorial. Thus, we can reduce the problem to the case where $X$ and $Y_{j}$ are proper.

If $G$ is an isomorphism, then we obtain (a). Thus, we may assume that $G$ is not an isomorphism. Then, we can take a $G$-exceptional curve $E_{j}$. We obtain

$$
\left(K_{Y_{j}}+E_{j}\right) \cdot E_{j} \leq\left(K_{Y_{j}}+\Delta_{j}\right) \cdot E_{j}=G^{*}\left(K_{X}+\Delta\right) \cdot E_{j}=0
$$


where $\Delta_{j}$ is defined by $K_{Y_{j}}+\Delta_{j}=G^{*}\left(K_{X}+\Delta\right)$. We may assume that $\left(K_{Y_{j}}+E_{j}\right) \cdot E_{j}=0$. In this case, the coefficient of $E_{j}$ in $\Delta_{j}$ is 1 .

First, assume that $\operatorname{Ex}(G)$ is reducible. Then, there exists a $G$-exceptional curve $E_{j}^{\prime}$ such that $E_{j} \cap E_{j}^{\prime} \neq \emptyset$. Then, we have

$$
\left(K_{Y_{j}}+E_{j}^{\prime}\right) \cdot E_{j}^{\prime}<\left(K_{Y_{j}}+\Delta_{j}\right) \cdot E_{j}^{\prime}=G^{*}\left(K_{X}+\Delta_{j}\right) \cdot E_{j}^{\prime}=0
$$

This is what we want to show.

Second, assume that $E:=\operatorname{Ex}(G)$ is irreducible. Since $\left(K_{Y_{j}}+E\right) \cdot E \leq$ 0 , we consider the two cases $\left(K_{Y_{j}}+E\right) \cdot E<0$ and $\left(K_{Y_{j}}+E\right) \cdot E=0$. If $\left(K_{Y_{j}}+E\right) \cdot E<0$, then this means (a). Assume that $\left(K_{Y_{j}}+E\right) \cdot E=0$. We show that $\Delta=0$. If $\Delta \neq 0$, then we have

$$
\left(K_{Y_{j}}+E\right) \cdot E<\left(K_{Y_{j}}+\Delta_{j}\right) \cdot E=0
$$

This means (b).

This theorem teaches us that non- $\mathbb{Q}$-factorial log canonical singularities are made by the case (b). Applying the same argument as above, we obtain the global version as follows.

Theorem 5.4. Let $(X, \Delta)$ be a log canonical surface, and let $f: Y \rightarrow X$ be the minimal resolution of $X$. Then, there exists a sequence of proper birational morphisms

$$
f: Y=: Y_{0} \stackrel{f_{0}}{\rightarrow} Y_{1} \stackrel{f_{1}}{\rightarrow} \cdots \stackrel{f_{m-1}}{\rightarrow} Y_{m}=: Z \stackrel{g}{\rightarrow} X
$$

with the following properties.

(1) Each $Y_{i}$ is a normal $\mathbb{Q}$-factorial surface.

(2) Each $f_{i}$ is a proper birational morphism, and $E_{i}:=\operatorname{Ex}\left(f_{i}\right)$ is an irreducible curve.

(3) Each $E_{i}$ satisfies $\left(K_{Y_{i}}+E_{i}\right) \cdot E_{i}<0$.

(4) Either (a) or (b) holds:

(a) $g$ is an isomorphism;

(b) $g(\operatorname{Ex}(g)) \cap \operatorname{Supp} \Delta=\emptyset$, and, for every point $Q \in g(\operatorname{Ex}(g)), g^{-1}(Q)=$ : $E$ is a proper irreducible curve such that $\left(K_{Y}+E\right) \cdot E=0$.

In particular, $K_{X}$ and all prime components of $\Delta$ are $\mathbb{Q}$-Cartier.

Proof. This follows from the same argument as for Theorem 5.3.

REMARK 5.5. By Theorem B.4, we see that $Z$ has, at worst, rational singularities. But we do not use this fact in this paper. 


\subsection{Minimal model theory for log canonical surfaces}

In this section, we consider the minimal model theory for log canonical surfaces. We have already proved the cone theorem in Section 3.2. Thus, let us consider the contraction theorem.

ThEOREM 5.6 (Contraction theorem). Let $(X, \Delta)$ be a projective log canonical surface, and let $R=\mathbb{R}_{\geq 0}[C]$ be a $\left(K_{X}+\Delta\right)$-negative extremal ray. Then there exists a morphism $\phi_{R}: X \rightarrow Y$ to a projective variety $Y$ with the following properties:

(1) let $C^{\prime}$ be a curve on $X$, and then $\phi_{R}\left(C^{\prime}\right)$ is one point if and only if $\left[C^{\prime}\right] \in R$;

(2) $\phi_{*}\left(\mathcal{O}_{X}\right)=\mathcal{O}_{Y}$

(3) if $L$ is a line bundle with $L \cdot C=0$, then $n L=\left(\phi_{R}\right)^{*} L_{Y}$ for some line bundle $L_{Y}$ on $Y$ and for some positive integer $n$;

(4) $\rho(Y)=\rho(X)-1$;

(5) $\left(Y,\left(\phi_{R}\right)_{*}(\Delta)\right)$ is a log canonical surface if $\operatorname{dim} Y=2$.

Proof of the case where $C^{2}>0$. First, we prove that there exists a curve $D$ in $X$ such that $D$ is Cartier, $D$ is ample, and $\mathbb{R}_{\geq 0}[C]=\mathbb{R}_{\geq 0}[D]$. Since $X$ is a projective normal surface, we can apply Bertini's theorem. Then the complete linear system of a very ample divisor has a smooth member $D$ such that $D \cap \operatorname{Sing}(X)=\emptyset$. Note that $D$ is a Cartier divisor. Let $f: X^{\prime} \rightarrow X$ be the minimal resolution, and let $D^{\prime}$ be the proper transform of $D$. Since $f^{*}(C)$ is a nef and big divisor, we obtain

$$
n f^{*}(C) \sim D^{\prime}+E
$$

for some effective divisor $E$ and some positive integer $n$. By sending this equation by $f_{*}$, we obtain

$$
n C \sim D+f_{*}(E)
$$

Since $\mathbb{R}_{\geq 0}[C]$ is extremal, we have $\mathbb{R}_{\geq 0}[C]=\mathbb{R}_{\geq 0}[D]$. Thus, we obtain $\rho(X)=1$, because we can apply the same argument as the one in the proof of Theorem 3.21. Set $Y:=\operatorname{Spec} k$. Then $\phi_{R}: X \rightarrow Y$ satisfies (1), (2), and (4). We want to prove (3). This follows from Lemma 5.7 because $K_{X}+\Delta$ is antiample.

LEMMA 5.7. Let $(X, \Delta)$ be a projective log canonical surface. Let $L$ be a nef line bundle such that $L-\left(K_{X}+\Delta\right)$ is ample. Then, $L$ is semiample. 
Proof. By Bertini's theorem, there exists a smooth curve $C$ such that

$$
n L-n\left(K_{X}+\Delta\right) \sim C
$$

$C \cap \operatorname{Sing}(X)=\emptyset$, and $C$ is not a component of $\Delta$. Let $f: X^{\prime} \rightarrow X$ be the minimal resolution, and let $C^{\prime}$ be the proper transform of $C$. Then we obtain

$$
n f^{*}(L)-n f^{*}\left(K_{X}+\Delta\right) \sim f^{*}(C)
$$

Since $f^{*}(C)=C^{\prime}$, we have

$$
f^{*}(L) \sim_{\mathbb{Q}} K_{X^{\prime}}+\Delta^{\prime}+\frac{1}{n} C^{\prime}
$$

where $\Delta^{\prime}$ is defined by $K_{X^{\prime}}+\Delta^{\prime}=f^{*}\left(K_{X}+\Delta\right)$. Since $\Delta^{\prime}+(1 / n) C^{\prime}$ is a boundary, $f^{*}(L)$ is semiample by Theorems 3.41 and 4.14 . Therefore, so is $L$.

In the proof of the case where $C^{2} \leq 0$ in Theorem 3.21, we use only the assumption of $\mathbb{Q}$-factoriality in the form that $K_{X}$ and $C$ are $\mathbb{Q}$-Cartier and $K_{X}+\Delta$ is $\mathbb{R}$-Cartier. Since $K_{X}$ is $\mathbb{Q}$-Cartier and $K_{X}+\Delta$ is $\mathbb{R}$-Cartier by Theorem 5.4, it is sufficient to prove that $C$ is $\mathbb{Q}$-Cartier.

Proof of the case where $C^{2}=0$. It is sufficient to prove that $\mathbb{R}_{\geq 0}[C]=$ $\mathbb{R}_{\geq 0}[D]$ for some $\mathbb{Q}$-Cartier curve $D$. Let $f: X^{\prime} \rightarrow X$ be the minimal resolution. Since $f^{*}(C)^{2}=0$ and $f^{*}(C) \cdot K_{X^{\prime}}<0$, we obtain $\kappa\left(X^{\prime}, f^{*}(C)\right)=1$. Therefore, $f^{*}(C)$ is semiample by Proposition 3.23. We consider the fibration $\pi: X^{\prime} \rightarrow B$ obtained by the complete linear system $\left|n f^{*}(C)\right|$ for some $n \gg 0$. For an arbitrary $f$-exceptional curve $E$, we have $E \cdot f^{*}(C)=0$. This means that an arbitrary exceptional curve is in some fiber of $\pi$. Thus, there exists an integral fiber $D^{\prime}$ of $\pi$ with $D^{\prime} \cap \operatorname{Ex}(f)=\emptyset$ by Proposition 5.8. This means that $f\left(D^{\prime}\right)=D$ is Cartier and that $n C \equiv D$. This is what we want to show.

Proposition 5.8. Let $\pi: X \rightarrow S$ be a dominant morphism from a normal surface $X$ to a curve $S$ with $\pi_{*} \mathcal{O}_{X}=\mathcal{O}_{S}$. Then there exists a nonempty open subset $S^{\prime}$ in $S$ such that all scheme-theoretic fibers of $\left.\pi\right|_{\pi^{-1}\left(S^{\prime}\right)}: \pi^{-1}\left(S^{\prime}\right) \rightarrow S^{\prime}$ are integral.

Proof. See, for example, [Bă1, Corollary 7.3]. 
For the proof of the case where $C^{2}<0$, we consider the relation between the non- $\mathbb{Q}$-factorial log canonical singularities and extremal curves $C$ with $C^{2}<0$. Since we want to prove that $C$ is $\mathbb{Q}$-Cartier, it is necessary to consider the case where $C$ passes through the singular points of (b) in Theorem 5.3. The following lemma demonstrates that these singularities are actually $\mathbb{Q}$-factorial.

Lemma 5.9. Let $(X, \Delta=0)$ be a local situation of a log canonical surface, and let $x$ be the singular point of $X$. Assume that this singularity is (b) in Theorem 5.3. If a proper curve $C$ in $X$ satisfies $C \cdot K_{X}<0, C^{2}<0$, and $x \in C$, then $X$ is $\mathbb{Q}$-factorial.

Proof. We use the notation in (b) of Theorem 5.4. It is sufficient to prove that $E \simeq \mathbb{P}^{1}$ by Propositions 3.25 and 3.26. Let $C_{Z}$ be the proper transform of $C$. Then, we obtain

$$
\begin{gathered}
C_{Z}^{2} \leq C_{Z} \cdot g^{*}(C)=C^{2}<0 \\
C_{Z} \cdot K_{Z} \leq C_{Z} \cdot\left(K_{Z}+E\right)=C_{Z} \cdot g^{*}\left(K_{X}\right)=C \cdot K_{X}<0 .
\end{gathered}
$$

Thus, we obtain $C_{Z} \simeq \mathbb{P}^{1}$, and $C_{Z}$ is a curve generating a $K_{Z}$-negative extremal ray. Let $\phi: Z \rightarrow Z^{\prime}$ be the contraction of $C_{Z}$. Since $\phi: E \rightarrow \phi(E)=$ : $E^{\prime}$ is a birational morphism, it is sufficient to prove that $E^{\prime} \simeq \mathbb{P}^{1}$. We would like to prove that

$$
\left(K_{Z^{\prime}}+E^{\prime}\right) \cdot E^{\prime}<0 .
$$

Let us consider the discrepancy $d$ defined by

$$
K_{Z}+E=\phi^{*}\left(K_{Z^{\prime}}+E^{\prime}\right)+d C_{Z} .
$$

Here, by taking the intersection with $E$, we obtain

$$
0=\left(K_{Z^{\prime}}+E^{\prime}\right) \cdot E^{\prime}+d C_{Z} \cdot E
$$

by $\left(K_{Z}+E\right) \cdot E=0$. By $x \in C$, we see that $C_{Z} \cdot E$ is a positive number. Thus, it is sufficient to prove that $d$ is a positive number. The inequality

$$
0>K_{X} \cdot C=g^{*}\left(K_{X}\right) \cdot C_{Z}=\left(K_{Z}+E\right) \cdot C_{Z}=d C_{Z}^{2}
$$

shows that $d$ is positive.

Proposition 5.10. Let $(X, \Delta)$ be a log canonical surface. If a proper curve $C$ in $X$ satisfies $C \cdot\left(K_{X}+\Delta\right)<0$ and $C^{2}<0$, then $C$ is $\mathbb{Q}$-Cartier. 
Proof. By Theorem 5.3 and Lemma 5.9, $C$ passes through only $\mathbb{Q}$-factorial points.

Thus, we complete the proof of the Theorem 5.6. Next, we consider the abundance theorem. But this immediately follows from the $\mathbb{Q}$-factorial case.

Theorem 5.11. Let $(X, \Delta)$ be a proper log canonical surface. If $K_{X}+\Delta$ is nef, then $K_{X}+\Delta$ is semiample.

Proof. Take the minimal resolution and apply Theorems 3.41 and 4.14.

\section{§6. Relativization}

\subsection{Relative cone theorem}

In this section, we consider the relativization of the cone theorem. But this is not difficult by the following proposition.

Proposition 6.1. Let $\pi: X \rightarrow S$ be a proper morphism from a normal surface $X$ to a variety $S$. If $\operatorname{dim} \pi(X) \geq 1$, where $\pi(X)$ is the schemetheoretic image of $\pi$, then we have

$$
\overline{N E}(X / S)=N E(X / S)=\sum_{\text {finite }} \mathbb{R}_{\geq 0}\left[C_{i}\right]
$$

Moreover, the Stein factorization $\pi: X \stackrel{\theta}{\rightarrow} T \rightarrow S$ satisfies one of the following assertions.

(1-irr) If $\operatorname{dim} \pi(X)=1$ and all fibers of $\theta$ are irreducible, then $N E(X / S)=$ $\mathbb{R}_{\geq 0}[C]$ and $C^{2}=0$. In particular, $\rho(X / S)=1$.

(1-red) If $\operatorname{dim} \pi(X)=1$ and $\theta$ has at least one reducible fiber, then each $C_{i}$ has negative self-intersection number.

(2) If $\operatorname{dim} \pi(X)=2$, then each $C_{i}$ has negative self-intersection number.

Proof. Note that $\operatorname{dim} \pi(X)=\operatorname{dim} T$ and that $N E(X / S)=N E(X / T)$.

(1-irr) All fibers are numerically equivalent. This is what we want to show.

(1-red) By Proposition 5.8, general fibers of $\theta$ are irreducible. Therefore, there are only finitely many reducible fibers. Since all fibers are numerically equivalent, $N E(X / S)$ is generated by the curves in the reducible fibers. Because all fibers of $\theta$ are connected, curves in reducible fibers have a negative self-intersection number.

(2) By $\theta_{*} \mathcal{O}_{X}=\mathcal{O}_{T}$, we see that $\theta$ is birational. Since the exceptional locus is a closed set, only finitely many curves are contracted by $\theta$. Each contracted curve has negative self-intersection number. 
Using this proposition, we obtain the following relative cone theorem.

TheOREM 6.2. Let $\pi: X \rightarrow S$ be a projective morphism from a normal surface $X$ to a variety $S$. Let $\Delta$ be an effective $\mathbb{R}$-divisor such that $K_{X}+\Delta$ is $\mathbb{R}$-Cartier. Let $\Delta=\sum b_{i} B_{i}$ be the prime decomposition. Let $H$ be an $\mathbb{R}$ Cartier $\pi$-ample $\mathbb{R}$-divisor on $X$. Then the following assertions hold:

(1) $\overline{N E}(X / S)=\overline{N E}(X / S)_{K_{X}+\Delta \geq 0}+\sum \mathbb{R}_{\geq 0}\left[C_{i}\right]$;

(2) $\overline{N E}(X / S)=\overline{N E}(X / S)_{K_{X}+\Delta+H \geq 0}+\sum_{\text {finite }} \mathbb{R}_{\geq 0}\left[C_{i}\right]$;

(3) each $C_{i}$ in (1) and (2) is rational or $C_{i}=B_{j}$ for some $B_{j}$ with $B_{j}^{2}<0$;

(4) there exists a positive integer $L(X, S, \Delta)$ such that each $C_{i}$ in (1) and (2) satisfies $0<-C_{i} \cdot\left(K_{X}+\Delta\right) \leq L(X, S, \Delta)$.

Proof. If $\operatorname{dim} \pi(X)=0$, then the assertion follows from Theorem 3.13. If $\operatorname{dim} \pi(X) \geq 1$, then assertions (1), (2), and (4) immediately follow from Proposition 6.1. We prove (3). Let $C$ be a $\left(K_{X}+\Delta\right)$-negative proper curve which generates an extremal ray, and $\pi(C)$ is one point. We may assume that $C \neq B_{j}$ for all $B_{j}$ with $B_{j}^{2}<0$. Take the Stein factorization of $\pi$ :

$$
\pi: X \stackrel{\theta}{\rightarrow} T \rightarrow S
$$

Let us take the Nagata compactification of $T$ and its normalization $\bar{T}$. Moreover, take the normalization $\bar{X}$ of a compactification of $X \rightarrow \bar{T}$. We obtain the following commutative diagram:

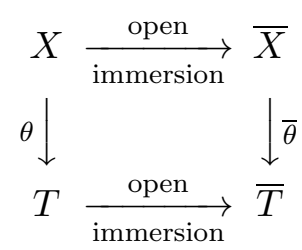

In $\bar{X}$, we can apply (BB2) in the sense of Definition 3.6 to $C$. Then we obtain

$$
p^{n} C \equiv_{\mathrm{Mum}} \alpha C^{\prime}+Z
$$

for a positive integer $n$, a nonnegative integer $\alpha$, a curve $C^{\prime}$, and a sum of rational curves $Z$. We consider the two cases $\operatorname{dim} T=1$ and $\operatorname{dim} T=2$.

Assume that $\operatorname{dim} T=1$. Take an ample divisor $A$ on $\bar{T}$. Since $C \cdot \bar{\theta}^{*} A=0$, the prime components of $Z$ must be $\bar{\theta}$-vertical. In advance, let $c_{0} \in C$ be a point, in the notation of Definition 3.6, such that $c_{0}$ is not contained in any curve $C^{\prime \prime} \neq C$ which is contained in the fiber containing $C$. Then, there 
exists a prime component $Z_{j}$ of $Z$ with $c_{0} \in Z_{j}$. Here $Z_{j}$ must be $C$. In particular, $C$ is rational, and this is what we want to show.

Assume that $\operatorname{dim} T=2$. Then, $\bar{T}$ is a proper normal surface. Since $\bar{\theta}_{*}\left(\alpha C^{\prime}+Z\right) \equiv_{\text {Mum }} 0$, each prime component of $Z$ is $\bar{\theta}$-exceptional. The remaining proof is the same as the case of $\operatorname{dim} T=1$.

We give an upper bound $L(X, S, \Delta)$ in the case where $\Delta$ is an $\mathbb{R}$-boundary.

Proposition 6.3. Let $\pi: X \rightarrow S$ be a projective morphism from a normal surface $X$ to a variety $S$. Let $\Delta$ be an $\mathbb{R}$-boundary such that $K_{X}+\Delta$ is $\mathbb{R}$-Cartier. If $R$ is a $\left(K_{X}+\Delta\right)$-negative extremal ray of $\overline{N E}(X / S)$, then $R=\mathbb{R}_{\geq 0}[C]$, where $C$ is a rational curve such that $-\left(K_{X}+\Delta\right) \cdot C \leq 3$.

Proof. If $\operatorname{dim} \pi(X)=0$, then the assertion follows from Proposition 3.15. Thus, we assume that $\operatorname{dim} \pi(X) \geq 1$. We can write $R=\mathbb{R}_{\geq 0}[C]$ for some curve $C$. We show that $C$ satisfies the desired properties. By $\operatorname{dim} \pi(X) \geq 1$ and Proposition 6.1, we see that $C^{2} \leq 0$. Then, by Lemma 3.9, we have

$$
-\left(K_{X}+\Delta\right) \cdot C \leq 2
$$

Since

$$
\left(K_{X}+C\right) \cdot C \leq\left(K_{X}+\Delta\right) \cdot C<0
$$

by Lemma 3.16, we see that $C$ is rational.

\subsection{Relative contraction theorem}

In this section, we consider the relativization of the contraction theorem.

Theorem 6.4. Let $\pi: X \rightarrow S$ be a projective morphism from a normal surface $X$ to a variety $S$. Let $\Delta$ be an $\mathbb{R}$-divisor. Moreover, one of the following conditions holds:

(QF) $X$ is $\mathbb{Q}$-factorial, and $\Delta$ is an $\mathbb{R}$-boundary;

(FP) $k=\overline{\mathbb{F}}_{p}$, and $\Delta$ is an effective $\mathbb{R}$-divisor;

(LC) $(X, \Delta)$ is a log canonical surface.

Let $R=\mathbb{R}_{\geq 0}[C]$ be a $\left(K_{X}+\Delta\right)$-negative extremal ray in $\overline{N E}(X / S)$. Then there exists a surjective $S$-morphism $\phi_{R}: X \rightarrow Y$ to a variety $Y$ projective over $S$ with the following properties:

(1) let $C^{\prime}$ be a curve on $X$, and then $\phi_{R}\left(C^{\prime}\right)$ is one point if and only if $\left[C^{\prime}\right] \in R$

(2) $\left(\phi_{R}\right)_{*}\left(\mathcal{O}_{X}\right)=\mathcal{O}_{Y} ;$ 
(3) if $L$ is an invertible sheaf with $L \cdot C=0$, then $n L=\left(\phi_{R}\right)^{*} L_{Y}$ for some invertible sheaf $L_{Y}$ on $Y$ and for some positive integer $n$;

(4) $\rho(Y / S)=\rho(X / S)-1$;

(5) if $\operatorname{dim} Y=2$, then $Y$ is $\mathbb{Q}$-factorial (resp., $\left(Y,\left(\phi_{R}\right)_{*}(\Delta)\right)$ is log canonical) in the case of $(Q F)$ (resp., $(L C)$ ).

These three proofs of (QF), (FP), and (LC) are the same essentially. Thus, we prove only the case when $(\mathrm{QF})$.

Proof of Theorem 6.4. Let $\theta: X \rightarrow T$ be the Stein factorization of $\pi$. We see that $\operatorname{dim} T=0, \operatorname{dim} T=1$, or $\operatorname{dim} T=2$. But the case $\operatorname{dim} T=0$ follows from Theorem 3.21. Thus, we may assume that $\operatorname{dim} T=1$ or $\operatorname{dim} T=2$.

Now let us take the compactification. First, take the Nagata compactification of $T$ and its normalization $\bar{T}$. Second, take the compactification $\bar{X}$ of $X \rightarrow \bar{T}$. Moreover, if necessary, replace it by its normalization and a resolution of the singular locus in $\bar{X} \backslash X$. We obtain the following commutative diagram:

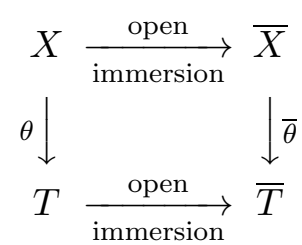

Then, $\bar{X}$ is projective normal $\mathbb{Q}$-factorial and $\bar{T}$ is proper normal. Let $\bar{\Delta}$ be the $\mathbb{R}$-boundary such that its restriction to $X$ is $\Delta$ and $\bar{\Delta}$ has no prime components contained in $\bar{X} \backslash X$.

Assume that $C^{2}<0$. This follows from Theorem 3.21 because $C$ is a $\left(K_{\bar{X}}+\bar{\Delta}\right)$-negative extremal curve in the cone of the absolute case $\overline{N E}(\bar{X})$.

Assume that $C^{2} \geq 0$. Then, by Proposition 6.1 , we see that $\rho(X / T)=1$ and that $\operatorname{dim} T=1$. Set $Y:=T$. The assertions (1), (2), and (4) are trivial. We want to prove (3). Note that all fibers of $\theta$ are irreducible but that the compactification $\bar{\theta}$ may have reducible fiber $G=\sum G_{i}$. Then, by

$$
0>\left(K_{\bar{X}}+\bar{\Delta}\right) \cdot G=\left(K_{\bar{X}}+\bar{\Delta}\right) \cdot \sum G_{i},
$$

we obtain $0>\left(K_{\bar{X}}+\bar{\Delta}\right) \cdot G_{i}$ for some irreducible component $G_{i}$ of the fiber $G$. Thus, by Theorem 3.21, we may assume that all fibers of $\bar{\theta}$ are irreducible. Therefore, each fiber $F$ of $\bar{\theta}$ is $\left(K_{\bar{X}}+\bar{\Delta}\right)$-negative. It is sufficient to prove that $F$ generates an extremal ray of $\overline{N E}(\bar{X})$. By Theorem 3.13, we have

$$
F \equiv D+\sum r_{i} C_{i}
$$


where $D \in \overline{N E}(\bar{X})_{K_{\bar{X}}+\bar{\Delta} \geq 0}, r_{i} \in \mathbb{R}_{\geq 0}$, and each $C_{i}$ generates a $\left(K_{\bar{X}}+\bar{\Delta}\right)$ negative extremal ray. Since $F$ is nef, we have $F \cdot D=F \cdot C_{i}=0$ for all $i$. Here recall that all fibers of $\bar{\theta}$ are irreducible. This means that $C_{i}$ is some fiber with the reduced structure. Thus, we obtain $F \equiv q C_{i}$ for some positive number $q$, and $F$ generates an extremal ray.

Then, we obtain the minimal model program in full generality.

Theorem 6.5 (Minimal model program). Let $\pi: X \rightarrow S$ be a projective morphism from a normal surface $X$ to a variety $S$. Let $\Delta$ be an $\mathbb{R}$-divisor on $X$. Assume that one of the following conditions holds:

(QF) $X$ is $\mathbb{Q}$-factorial, and $0 \leq \Delta \leq 1$;

(FP) $k=\overline{\mathbb{F}}_{p}$, and $0 \leq \Delta$;

(LC) $(X, \Delta)$ is a log canonical surface.

Then, there exists a sequence of proper birational morphisms

$$
\begin{aligned}
& (X, \Delta)=:\left(X_{0}, \Delta_{0}\right) \stackrel{\phi_{0}}{\rightarrow}\left(X_{1}, \Delta_{1}\right) \stackrel{\phi_{1}}{\rightarrow} \ldots \stackrel{\phi_{s-1}}{\rightarrow}\left(X_{s}, \Delta_{s}\right)=:\left(X^{\dagger}, \Delta^{\dagger}\right), \\
& \quad \text { where }\left(\phi_{i-1}\right)_{*}\left(\Delta_{i-1}\right)=: \Delta_{i},
\end{aligned}
$$

with the following properties.

(1) Each $X_{i}$ is a normal surface, which is projective over $S$.

(2) Each $\left(X_{i}, \Delta_{i}\right)$ satisfies (QF), (FP), or (LC) according to the above assumption.

(3) For each $i, \operatorname{Ex}\left(\phi_{i}\right)=: C_{i}$ is a proper irreducible curve such that

$$
\left(K_{X_{i}}+\Delta_{i}\right) \cdot C_{i}<0
$$

and such that $C_{i}$ generates an extremal ray of $\overline{N E}(X / S)$.

(4) Let $\pi^{\dagger}: X^{\dagger} \rightarrow S$ be the $S$-scheme structure morphism; $\left(X^{\dagger}, \Delta^{\dagger}\right)$ satisfies one of the following conditions:

(a) $K_{X^{\dagger}}+\Delta^{\dagger}$ is $\pi^{\dagger}$-nef;

(b) there is a projective surjective $S$-morphism $\mu: X^{\dagger} \rightarrow Z$ to a smooth curve $Z$ such that $Z$ is projective over $S, \mu_{*} \mathcal{O}_{X^{\dagger}}=\mathcal{O}_{Z},-\left(K_{X}^{\dagger}+\Delta^{\dagger}\right)$ is $\mu$-ample, and $\rho\left(X^{\dagger} / Z\right)=1$;

(c) $X^{\dagger}$ is a projective surface, $-\left(K_{X^{\dagger}}+\Delta^{\dagger}\right)$ is ample, and $\rho\left(X^{\dagger}\right)=1$.

In case (a), we say that $\left(X^{\dagger}, \Delta^{\dagger}\right)$ is a minimal model of $(X, \Delta)$ over $S$.

In cases (b) and (c), we say that $\left(X^{\dagger}, \Delta^{\dagger}\right)$ is a Mori fiber space over $S$. 


\subsection{Relative abundance theorem}

In this section, we consider the relativization of the abundance theorem. To restate the problem from the absolute case to the relative case, let us consider the following lemma.

Lemma 6.6. Let $\pi: X \rightarrow S$ be a morphism from a projective normal $\mathbb{Q}$ factorial surface $X$ to a projective variety $S$. Let $\Delta$ be an $\mathbb{R}$-boundary on $X$. If $K_{X}+\Delta$ is $\pi$-nef, then there exists an ample line bundle $F$ on $S$ such that $\Delta+\pi^{*}(F) \sim_{\mathbb{R}} \Delta^{\prime}$ for some $\mathbb{R}$-boundary $\Delta^{\prime}$ and $K_{X}+\Delta^{\prime}$ is nef.

Proof. Take the Stein factorization of $\pi$

$$
\pi: X \stackrel{\theta}{\rightarrow} T \stackrel{\sigma}{\rightarrow} S
$$

Take an arbitrary ample line bundle $H$ on $S$. Since $\sigma$ is a finite morphism, $\sigma^{*}(H)$ is also ample. We may assume that $\sigma^{*}(H)$ is very ample by replacing $H$ with its multiple. Note that $\sigma^{*}(4 H)$ is very ample. We want to prove that $F:=4 H$ satisfies the assertion. If $\operatorname{dim} T=0$, then the assertion is obvious. Thus, we can consider the following two cases: (1) $\operatorname{dim} T=1$ and (2) $\operatorname{dim} T=2$.

(1) Assume that $\operatorname{dim} T=1$. In this case, $T$ is a smooth projective curve, and general fibers of $\theta$ are integral by Proposition 5.8. Thus, we can take a hyperplane section

$$
P_{1}+\cdots+P_{n}=G \in\left|\sigma^{*}(4 H)\right|
$$

such that $P_{i} \neq P_{j}$ for all $i \neq j, \theta^{-1}\left(P_{i}\right)$ is integral for each $i$, and $\theta^{-1}\left(P_{i}\right)$ is not a component of $\Delta$ for each $i$. Therefore, for an $\mathbb{R}$-boundary $\Delta^{\prime}$ defined by

$$
\Delta^{\prime}:=\Delta+\theta^{*}(G),
$$

$K_{X}+\Delta^{\prime}$ is nef by Theorem 3.13 and Proposition 3.15.

(2) Assume that $\operatorname{dim} T=2$. In this case, $T$ is a normal projective surface, and $\theta$ is birational. By Bertini's theorem, we can take an irreducible smooth hyperplane section $G \in\left|\sigma^{*}(4 H)\right|$ such that $\operatorname{Supp} G \cap \theta(\operatorname{Ex}(\theta))=\emptyset$ and $G$ is not a component of $\theta_{*}(\Delta)$. Then, $\Delta^{\prime}:=\Delta+\theta^{*}(G)$ is an $\mathbb{R}$-boundary, and $K_{X}+\Delta^{\prime}$ is nef by Theorem 3.13 and Proposition 3.15.

We can prove the relative abundance theorem for $\mathbb{Q}$-factorial surfaces with $\mathbb{R}$-boundary. 
TheOREM 6.7. Let $\pi: X \rightarrow S$ be a projective morphism from a normal $\mathbb{Q}$-factorial surface $X$ to a variety $S$. Let $\Delta$ be an $\mathbb{R}$-boundary. If $K_{X}+\Delta$ is $\pi$-nef, then $K_{X}+\Delta$ is $\pi$-semiample.

Proof. We may assume that $S$ is affine. Moreover, by taking Nagata's compactification, we may assume that $S$ is projective and that $X$ is projective $\mathbb{Q}$-factorial. Note that the hypothesis of $\pi$-nefness may break up by taking the compactification. But, by running a $\left(K_{X}+\Delta\right)$-minimal model program over $S$, we may assume this hypothesis. Thus, we can apply Lemma 6.6. Note that $F$ and $\Delta^{\prime}$ are the same notation as Lemma 6.6. Since $K_{X}+\Delta^{\prime}$ is nef, $K_{X}+\Delta^{\prime}$ is semiample by the abundance theorem of the absolute case. By $K_{X}+\Delta^{\prime} \sim_{\mathbb{R}} K_{X}+\Delta+\pi^{*}(F), K_{X}+\Delta$ is $\pi$-semiample.

We obtain the following theorem by applying the same argument.

Theorem 6.8. Let $\pi: X \rightarrow S$ be a projective morphism from a normal surface $X$ to a variety $S$, defined over $\overline{\mathbb{F}}_{p}$. Let $\Delta$ be an effective $\mathbb{R}$-divisor. If $K_{X}+\Delta$ is $\pi$-nef, then $K_{X}+\Delta$ is $\pi$-semiample.

Proof. We can apply the same proof as the one for Theorem 6.7.

The $\log$ canonical case immediately follows from the $\mathbb{Q}$-factorial case.

TheOREm 6.9. Let $\pi: X \rightarrow S$ be a projective morphism from a log canonical surface $(X, \Delta)$ to a variety $S$. If $K_{X}+\Delta$ is $\pi$-nef, then $K_{X}+\Delta$ is $\pi$-semiample.

Proof. Take the minimal resolution, and apply Theorem 6.7.

We summarize the results obtained in this section.

Corollary 6.10. Let $\pi: X \rightarrow S$ be a projective morphism from a normal surface $X$ to a variety $S$. Let $\Delta$ be an $\mathbb{R}$-divisor on $X$. Assume that one of the following conditions holds:

(QF) $X$ is $\mathbb{Q}$-factorial, and $0 \leq \Delta \leq 1$;

(FP) $k=\overline{\mathbb{F}}_{p}$, and $0 \leq \Delta$;

(LC) $(X, \Delta)$ is a log canonical surface.

If $K_{X}+\Delta$ is $\pi$-nef, then $K_{X}+\Delta$ is $\pi$-semiample. 


\section{Appendix A. Base-point-free theorem}

In this section, we consider the base-point-free theorem. First, we prove the following nonvanishing theorem.

TheOREm A.1. Let $X$ be a projective normal $\mathbb{Q}$-factorial surface, and let $\Delta$ be a $\mathbb{Q}$-boundary. Let $D$ be a nef Cartier divisor. Assume that $D-$ $\left(K_{X}+\Delta\right)$ is nef and big and that $\left(D-\left(K_{X}+\Delta\right)\right) \cdot C>0$ for every curve $C \subset \operatorname{Supp}\llcorner\Delta\lrcorner$. Then $\kappa(X, D) \geq 0$.

Proof. If $k=\overline{\mathbb{F}}_{p}$, then the assertion follows from Theorem 4.15. Thus, we may assume that $k \neq \overline{\mathbb{F}}_{p}$.

Assume that $\kappa(X, D)=-\infty$, and we derive a contradiction. Let $f: X^{\prime} \rightarrow$ $X$ be the minimal resolution, let $K_{X^{\prime}}+\Delta^{\prime}=f^{*}\left(K_{X}+\Delta\right)$, and let $D^{\prime}=f^{*} D$.

SteP 1. We may assume that $\kappa\left(X^{\prime}, K_{X^{\prime}}\right)=-\infty$.

Indeed, we have $\kappa\left(X^{\prime}, K_{X^{\prime}}\right) \leq \kappa\left(X^{\prime}, K_{X^{\prime}}+\Delta^{\prime}\right)=\kappa\left(X, K_{X}+\Delta\right)=-\infty$. Note that, if $\kappa\left(X, K_{X}+\Delta\right) \geq 0$, then we have $\kappa(X, D)=\kappa\left(X, D-\left(K_{X}+\right.\right.$ $\left.\Delta)+\left(K_{X}+\Delta\right)\right) \geq 0$. This is what we want to show.

Step 2. In this step, we show that $h^{2}\left(X^{\prime}, D^{\prime}\right)=0$.

By Serre duality, we have

$$
\begin{aligned}
h^{2}\left(X^{\prime}, D^{\prime}\right) & =h^{0}\left(X^{\prime}, K_{X^{\prime}}-D^{\prime}\right) \quad \text { and } \\
\kappa\left(X^{\prime}, K_{X^{\prime}}-D^{\prime}\right) & \leq \kappa\left(X^{\prime}, K_{X^{\prime}}+\Delta^{\prime}-D^{\prime}\right)=\kappa\left(X, K_{X}+\Delta-D\right)=-\infty
\end{aligned}
$$

because $-\left(K_{X}+\Delta-D\right)$ is nef and big.

SteP 3. In this step, we prove that $X^{\prime}$ is an irrational ruled surface.

It is sufficient to prove that $\chi\left(\mathcal{O}_{X^{\prime}}\right) \leq 0$. Since $h^{0}\left(X^{\prime}, D^{\prime}\right)=h^{2}\left(X^{\prime}, D^{\prime}\right)=$ 0 , by the Riemann-Roch theorem, we obtain

$$
-h^{1}\left(X^{\prime}, D^{\prime}\right)=\chi\left(\mathcal{O}_{X^{\prime}}\right)+\frac{1}{2} D^{\prime} \cdot\left(D^{\prime}-K_{X^{\prime}}\right) .
$$

Since

$$
\begin{aligned}
& D^{\prime} \cdot\left(D^{\prime}-K_{X^{\prime}}\right)=D \cdot\left(D-K_{X}\right) \quad \text { and } \\
& \kappa\left(X, D-K_{X}\right) \geq \kappa\left(X, D-\left(K_{X}+\Delta\right)\right)=2,
\end{aligned}
$$

we have $D^{\prime} \cdot\left(D^{\prime}-K_{X^{\prime}}\right) \geq 0$ by the nefness of $D$. Therefore, we get $0 \geq$ $-h^{1}\left(X^{\prime}, D^{\prime}\right) \geq \chi\left(\mathcal{O}_{X^{\prime}}\right)$. 
Let $\pi: X^{\prime} \rightarrow Z$ be its ruling. By Theorem 3.20, $\pi$ factors through $X$.

STEP 4. We reduce the proof to the case where there is no curve $C$ in $X$ such that $D \cdot C=0$ and $C^{2}<0$.

Let $C$ be such a curve. We have $\left(K_{X}+C\right) \cdot C<0$ by the assumption. (Indeed, if $C \subset \operatorname{Supp}\llcorner\Delta\lrcorner$, then

$$
-\left(K_{X}+C\right) \cdot C \geq-\left(K_{X}+\Delta\right) \cdot C=\left(D-\left(K_{X}+\Delta\right)\right) \cdot C>0 .
$$

If $C \not \subset \operatorname{Supp}\llcorner\Delta\lrcorner$, then

$$
\left.-\left(K_{X}+C\right) \cdot C>-\left(K_{X}+\Delta\right) \cdot C=\left(D-\left(K_{X}+\Delta\right)\right) \cdot C \geq 0 .\right)
$$

This shows that $C=\mathbb{P}^{1}$ and that $C$ is contractible. Moreover, this induces a contraction map $g: X \rightarrow Y$ to a $\mathbb{Q}$-factorial surface $Y$, and the irrationality of $X$ shows that $\pi$ factors through $Y$. Let $g_{*} D=D_{Y}$, and let $g_{*}(\Delta)=\Delta_{Y}$. Then, we have $K_{X}+\Delta=g^{*}\left(K_{Y}+\Delta_{Y}\right)+a C$ for some nonnegative rational number $a$. Therefore, it is easy to see that $Y$ has all the assumptions of $X$.

STEP 5. We reduce the proof to the case where $K_{X}+\Delta$ is not nef. In particular, there is at least one $\left(K_{X}+\Delta\right)$-negative extremal ray.

If $K_{X}+\Delta$ is nef, then $D=D-\left(K_{X}+\Delta\right)+\left(K_{X}+\Delta\right)$ is nef and big, and this is what we want to show. Thus, we may assume that $K_{X}+\Delta$ is not nef.

STEP 6. We reduce the proof to the case where $D \equiv 0$.

The nefness of $D$ and $\kappa(X, D)=-\infty$ show that $D^{2}=0$. Since $D$ and $D-\left(K_{X}+\Delta\right)$ are nef, we have $\left(D-\left(K_{X}+\Delta\right)\right) \cdot D=-\left(K_{X}+\Delta\right) \cdot D \geq 0$. We consider the two cases $-\left(K_{X}+\Delta\right) \cdot D=0$ and $-\left(K_{X}+\Delta\right) \cdot D>0$. If $-\left(K_{X}+\Delta\right) \cdot D=0$, then we obtain $D \equiv 0$ by the bigness of $D-\left(K_{X}+\Delta\right)$. This is what we want to show. If $-\left(K_{X}+\Delta\right) \cdot D>0$, then we have $K_{X}$. $D<0$. Two conditions $K_{X} \cdot D<0$ and $D^{2}=0$ mean that $\kappa(X, D)=1$ by resolution and the Riemann-Roch theorem. This case is excluded.

SteP 7. By Steps 4 and 6 , there exists no curve $C$ with $C^{2}<0$. By Step 5 and the classification of extremal rays, we have $\rho(X) \leq 2$. Since there is a surjection $X \rightarrow Z$ to a curve $Z$, we have $\rho(X) \neq 1$. Thus, we obtain $\rho(X)=2$. Here, $-\left(K_{X}+\Delta\right)$ is ample because $-\left(K_{X}+\Delta\right)$ is nef and big and because of Step 4. Moreover, by Step 4, there are two extremal rays inducing the structure of the Mori fiber space to a curve. By Proposition 3.15, every extremal ray is generated by a rational curve. This contradicts the irrationality of $Z$. 
This completes the proof.

Using the nonvanishing theorem, we obtain the following base-point-free theorem.

TheOREM A.2. Let $X$ be a projective normal $\mathbb{Q}$-factorial surface, and let $\Delta$ be a $\mathbb{Q}$-boundary. Let $D$ be a nef Cartier divisor. Assume that $D$ $\left(K_{X}+\Delta\right)$ is nef and big and that $\left(D-\left(K_{X}+\Delta\right)\right) \cdot C>0$ for every curve $C \subset \operatorname{Supp}\llcorner\Delta\lrcorner$. Then $D$ is semiample.

Proof. By Theorem A.1, we may assume that $\kappa(X, D) \geq 0$. But by Proposition 3.23, we may assume that $\kappa(X, D)=0$ or 2 . By the same argument as Step 4 in the proof of Theorem A.1, we may assume that there is no curve $C$ in $X$ with $D \cdot C=0$ and $C^{2}<0$. Thus, if $\kappa(X, D)=2$, then $D$ is ample. This is what we want to show. Hence, the remaining case is $\kappa(X, D)=0$. We have linear equivalence to the effective divisor $n D \sim \sum d_{i} D_{i}$. Assume that $\sum d_{i} D_{i} \neq 0$, and let us get a contradiction. Since $D^{2}=0$ and $D$ is nef, we have $D \cdot D_{i}=0$ for all $i$. Moreover, we get $D_{i}^{2} \geq 0$ by the above reduction. Then, we obtain $D_{i}^{2}=D_{i} \cdot D=0$. Since $D-\left(K_{X}+\Delta\right)$ is nef and big, we have

$$
\left(D-\left(K_{X}+\Delta\right)\right) \cdot D_{i}=-\left(K_{X}+\Delta\right) \cdot D_{i}>0 \text {. }
$$

This means that $K_{X} \cdot D_{i}<0$. Then $D_{i}^{2}=0$ and $K_{X} \cdot D_{i}<0$ show that $\kappa\left(X, D_{i}\right)=1$ by taking a resolution and applying the Riemann-Roch theorem. This contradicts $\kappa(X, D)=0$.

The following example demonstrates that the base-point-free theorem does not hold only under the boundary condition.

EXAMPLE A.3. If $k \neq \overline{\mathbb{F}}_{p}$, then there exist a smooth projective surface $X$ over $k$, an elliptic curve $C$ in $X$, and a divisor $D$ such that $K_{X}+C=0$ and the divisor $D=D-\left(K_{X}+C\right)$ is nef and big but not semiample.

Construction. Let $X_{0}:=\mathbb{P}^{2}$, and let $C_{0}$ be an elliptic curve in $X_{0}$. Let $P_{1}, \ldots, P_{10}$ be 10 points which are linearly independent. Blow up these 10 points. We obtain the surface $X$, and let $C$ be the proper transform of $C_{0}$. Then $K_{X}+C=0$ and $C$ is not contractible by Answer 4.2 and its construction. On the other hand, take an ample divisor $H$, and let $D$ be the divisor $D:=H+q C$ with $(H+q C) \cdot C=0$. It is easy to check that $D$ is nef and big. Because $C$ is not contractible, $D$ is not semiample.

We can also prove a base-point-free theorem under the following assumption. 
TheOREm A.4. Let $X$ be a projective normal $\mathbb{Q}$-factorial surface, and let $\Delta$ be a $\mathbb{Q}$-boundary. Let $D$ be a nef Cartier divisor. Assume that $D-\left(K_{X}+\right.$ $\Delta)$ is semiample. Then $D$ is semiample.

Proof. Set $\kappa:=\kappa\left(X, D-\left(K_{X}+\Delta\right)\right)$. There are three cases: (0) $\kappa=0$, (1) $\kappa=1$, and $(2) \kappa=2$.

(0) Assume that $\kappa=0$. By the semiampleness, we obtain $D-\left(K_{X}+\right.$ $\Delta) \sim_{\mathbb{Q}} 0$. Thus, we can apply the abundance theorem to $D$. Then we obtain the desired result.

(1) Assume that $\kappa=1$. By the semiampleness, the complete linear system $\left|n\left(D-\left(K_{X}+\Delta\right)\right)\right|$ induces a morphism $\sigma: X \rightarrow B$ to a smooth projective curve. By Proposition 5.8, we can find a boundary

$$
\Delta^{\prime} \sim_{\mathbb{Q}} D-\left(K_{X}+\Delta\right)
$$

such that $\Delta+\Delta^{\prime}$ is a $\mathbb{Q}$-boundary. Thus, we can apply the abundance theorem to $K_{X}+\Delta+\Delta^{\prime}$.

(2) Assume that $\kappa=2$. The complete linear system $\left|n\left(D-\left(K_{X}+\Delta\right)\right)\right|$ induces a birational morphism $f: X \rightarrow Y$ to a normal projective surface. Since $n\left(D-\left(K_{X}+\Delta\right)\right)=f^{*}\left(H_{Y}\right), H_{Y}$ is an very ample line bundle on $Y$. By Bertini's theorem, we can find a member $G \in\left|H_{Y}\right|$ such that

$$
\Delta+\frac{1}{n} f^{*}(G)
$$

is a boundary. Thus, we can apply the abundance theorem.

\section{Appendix B. Rational singularities}

In this section, we consider the relation between the minimal model program and the rational singularities.

Definition B.1. Let $X$ be a normal surface, and let $f: Y \rightarrow X$ be a resolution of singularities. We say that $X$ has at worst rational singularities if $R^{1} f_{*} \mathcal{O}_{Y}=0$. This property is independent of the choice of resolutions of singularities.

If $X$ is a normal surface whose singularities are at worst rational, then $X$ is $\mathbb{Q}$-factorial by [L, Proposition 17.1]. Let us give an alternative proof of this result. 
Proposition B.2. Let $X$ be a normal surface. If $X$ has at worst rational singularities, then $X$ is $\mathbb{Q}$-factorial.

Proof. Note that, if $g: Z \rightarrow X$ is a proper birational morphism, and if $E$ is a $g$-exceptional curve, then $E \simeq \mathbb{P}^{1}$.

We may assume that $X$ is affine. Thus, we may assume that $X$ is projective. Let $f: Y \rightarrow X$ be the minimal resolution. Let $E$ be an $f$-exceptional curve. By Proposition 3.24, we can contract $E$, and we obtain

$$
f: Y \rightarrow Y^{\prime} \stackrel{f^{\prime}}{\rightarrow} X
$$

By Propositions 3.25 and $3.26, X^{\prime}$ is $\mathbb{Q}$-factorial. Assume that $f^{\prime}$ is not an isomorphism. Then we can take an $f^{\prime}$-exceptional curve $E^{\prime}$. By the same argument, we can contract $E^{\prime}$ to a $\mathbb{Q}$-factorial surface. Repeat the same procedure. Then, we see that $X$ is $\mathbb{Q}$-factorial.

The Kodaira vanishing theorem does not hold in positive characteristic. But we obtain the following relative vanishing theorem.

THEOREM B.3. Let $f: X \rightarrow Y$ be a proper birational morphism from a smooth surface $X$ to a normal surface $Y$. Let $L$ be a line bundle on $X$ such that

$$
L \equiv{ }_{f} K_{X}+E+N,
$$

where $E$ is an effective $f$-exceptional $\mathbb{R}$-boundary and $N$ is an $f$-nef $\mathbb{R}$ divisor. If $E_{i} \cdot N>0$ for every curve $E_{i}$ with $E_{i} \subset\llcorner E\lrcorner$, then $R^{1} f_{*}(L)=0$.

Proof. For a proof, see [KoK, Section 2.2].

In this paper, we often use the contraction of $\mathbb{P}^{1}$. For example, the minimal model program of Theorem 3.27 is the composition of the contractions of $C \simeq \mathbb{P}^{1}$ with $\left(K_{X}+C\right) \cdot C<0$. The following theorem shows that the $R^{1}$ of such contractions vanishes.

TheOREM B.4. Let $g: Y \rightarrow Z$ be a proper birational morphism between normal surfaces such that $C:=\operatorname{Ex}(g)$ is an irreducible curve. If $\left(K_{Y}+C\right)$. $C<0$, then $R^{1} g_{*}\left(\mathcal{O}_{Y}\right)=0$.

Proof. Let $f: X \rightarrow Y$ be the minimal resolution of $Y$, and let $C_{X}$ be the proper transform of $C$. Set $K_{X}+C_{X}+\Delta_{X}=f^{*}\left(K_{Y}+C\right)$. Then we have

$$
\left.{ }_{-\llcorner} \Delta_{X}\right\lrcorner=K_{X}+\left(\left\{\Delta_{X}\right\}+C_{X}\right)-f^{*}\left(K_{Y}+C\right) .
$$


We apply Theorem B.3 and obtain

$$
R^{1}(g \circ f)_{*} \mathcal{O}_{X}\left(-\left\llcorner\Delta_{X}\right\lrcorner\right)=0
$$

by $-f^{*}\left(K_{Y}+C\right) \cdot C_{X}>0$.

If $\left\llcorner\Delta_{X}\right\lrcorner=0$, then we obtain

$$
R^{1} g_{*}\left(\mathcal{O}_{Y}\right)=R^{1} g_{*}\left(f_{*} \mathcal{O}_{X}\right) \subset R^{1}(g \circ f)_{*}\left(\mathcal{O}_{X}\right)=0
$$

by the Grothendieck-Leray spectral sequence. Thus, we may assume that $\left\llcorner\Delta_{X}\right\lrcorner \neq 0$. Since

$$
0 \rightarrow \mathcal{O}_{X}\left(-\left\llcorner\Delta_{X}\right\lrcorner\right) \rightarrow \mathcal{O}_{X} \rightarrow \mathcal{O}_{\left\llcorner\Delta_{X}\right\lrcorner} \rightarrow 0
$$

we obtain

$$
\begin{gathered}
0 \rightarrow f_{*} \mathcal{O}_{X}\left(-\left\llcorner\Delta_{X}\right\lrcorner\right) \rightarrow \mathcal{O}_{Y} \rightarrow \mathcal{C} \rightarrow 0, \\
\mathcal{C} \subset f_{*} \mathcal{O}_{\left\llcorner\Delta_{X}\right\lrcorner},
\end{gathered}
$$

where $\mathcal{C}$ is the cokernel of $f_{*} \mathcal{O}_{X}\left(-\left\llcorner\Delta_{X}\right\lrcorner\right) \rightarrow \mathcal{O}_{Y}$. Since $f_{*} \mathcal{O}_{\left\llcorner\Delta_{X}\right\lrcorner}$ is a skyscraper sheaf, so is $\mathcal{C}$. Thus, we obtain

$$
R^{1} g_{*}\left(f_{*} \mathcal{O}_{X}\left(-\left\llcorner\Delta_{X}\right\lrcorner\right)\right) \rightarrow R^{1} g_{*}\left(\mathcal{O}_{Y}\right) \rightarrow R^{1} g_{*}(\mathcal{C})=0 .
$$

By the Grothendieck-Leray spectral sequence, we obtain

$$
R^{1} g_{*}\left(f_{*} \mathcal{O}_{X}\left(-\left\llcorner\Delta_{X}\right\lrcorner\right)\right) \subset R^{1}(g \circ f)_{*} \mathcal{O}_{X}\left(-\left\llcorner\Delta_{X}\right\lrcorner\right)=0 .
$$

Therefore, we have $R^{1} g_{*}\left(\mathcal{O}_{Y}\right)=0$.

As corollaries, we obtain the results on minimal models and canonical models for surfaces with rational singularities.

Corollary B.5. Let $\pi: X \rightarrow S$ be a projective morphism from a normal surface $X$ to a variety $S$. Let $\Delta$ be an $\mathbb{R}$-boundary. Assume that $X$ has at worst rational singularities. Then, the following assertions hold.

(1) The surface $X$ is $\mathbb{Q}$-factorial. In particular, by Theorem 6.5, we can run a $\left(K_{X}+\Delta\right)$-minimal model program over $S$

$$
\begin{aligned}
& (X, \Delta)=:\left(X_{0}, \Delta_{0}\right) \stackrel{\phi_{0}}{\rightarrow}\left(X_{1}, \Delta_{1}\right) \stackrel{\phi_{1}}{\rightarrow} \cdots \stackrel{\phi_{s-1}}{\rightarrow}\left(X_{s}, \Delta_{s}\right), \\
& \text { where }\left(\phi_{i-1}\right)_{*}\left(\Delta_{i-1}\right)=: \Delta_{i} .
\end{aligned}
$$


(2) Each $X_{i}$ has at worst rational singularities.

Proof. Assertion (1) follows from Proposition B.2. Each extremal contraction in a minimal model program of $(X, \Delta)$ satisfies the condition of Theorem B.4. This implies (2).

Corollary B.6. Let $\pi: X \rightarrow S$ be a projective morphism from a normal surface $X$ to a variety $S$. Let $\Delta$ be an $\mathbb{R}$-divisor such that $0 \leq \Delta<1$. If $X$ has at worst rational singularities, and if $K_{X}+\Delta$ is $\pi$-big, then the canonical model of $(X, \Delta)$ over $S$ has at worst rational singularities.

Proof. By Corollary B.5 we may assume that $K_{X}+\Delta$ is $\pi$-nef and $\pi$-big. If $\left(K_{X}+\Delta\right) \cdot C=0$ for some curve $C$ such that $\pi(C)$ is one point, then $\left(K_{X}+C\right) \cdot C<0$ because $0 \leq \Delta<1$. Therefore, we can contract this curve $C$, and $C$ satisfies the condition of Theorem B.4. Repeat this procedure and obtain the required assertion.

Acknowledgments. The author would like to thank Osamu Fujino and Kenji Matsuki for many comments and discussions. He also thanks Lucian Bădescu for many valuable comments and Atsushi Moriwaki for warm encouragement. Thanks are due the referees for many valuable comments and suggestions. The author's work was partially supported by the Japan Society for the Promotion of Science Fellowships for Young Scientists.

\section{REFERENCES}

[A] M. Artin, Some numerical criteria for contractability of curves on algebraic surfaces, Amer. J. Math. 84 (1962), 485-496. MR 0146182.

[Bă1] L. Bădescu, Algebraic Surfaces, Springer, New York, 2001. MR 1805816. DOI 10.1007/978-1-4757-3512-3.

[Bă2] , "On some contractibility criteria of curves on surfaces" in Sitzungsberichte der Berliner Mathematischen Gesellschaft, Berliner Math. Gesellschaft, Berlin, 2001, 41-51. MR 2091877.

[Bi] C. Birkar, On existence of log minimal models, II, J. Reine Angew. Math. 658 (2011), 99-113. MR 2831514. DOI 10.1515/CRELLE.2011.062.

[BM1] E. Bombieri and D. Mumford, "Enriques' classification of surfaces in char $p$, II" in Complex Analysis and Algebraic Geometry, Iwanami Shoten, Tokyo, 1977, $23-42$. MR 0491719.

[BM2] - Enriques' classification of surfaces in char p, III, Invent. Math. 35 (1976), 197-232. MR 0491720.

[CMM] P. Cascini, J. McKernan, and M. Mustaţă, The augmented base locus in positive characteristic, Proc. Edinb. Math. Soc. (2) 57 (2014), 79-87. MR 3165013. DOI 10.1017/S0013091513000916. 
[FJ] G. Frey and M. Jarden, Approximation theory and the rank of abelian varieties over large algebraic fields, Proc. Lond. Math. Soc. (3) 28 (1974), 112-128. MR 0337997.

[F1] O. Fujino, Fundamental theorems for the log minimal model program, Publ. Res. Inst. Math. Sci. 47 (2011), 727-789. MR 2832805. DOI 10.2977/PRIMS/50.

[F2] - Minimal model theory for log surfaces, Publ. Res. Inst. Math. Sci. 48 (2012), 339-371. MR 2928144. DOI 10.2977/PRIMS/71.

[FT] O. Fujino and H. Tanaka, On log surfaces, Proc. Japan Acad. Ser. A Math. Sci. 88 (2012), 109-114. MR 2989060. DOI 10.3792/pjaa.88.109.

[Fu] T. Fujita, Fractionally logarithmic canonical rings of algebraic surfaces, J. Fac. Sci. Univ. Tokyo Sect. IA Math. 30 (1984), 685-696. MR 0731524.

[H] R. Hartshorne, Algebraic Geometry, Grad. Texts in Math. 52, Springer, New York, 1977. MR 0463157.

[K] Y. Kawamata, Semistable minimal models of threefolds in positive or mixed characteristic, J. Algebraic Geom. 3 (1994), 463-491. MR 1269717.

[KMM] Y. Kawamata, K. Matsuda, and K. Matsuki, "Introduction to the minimal model problem" in Algebraic Geometry (Sendai, 1985), Adv. Stud. Pure Math. 10, North-Holland, Amsterdam, 1987, 283-360. MR 0946243.

[Ke1] S. Keel, Basepoint freeness for nef and big line bundles in positive characteristic, Ann. of Math. (2) 149 (1999), 253-286. MR 1680559. DOI 10.2307/121025.

[Ke2] - Polarized pushouts over finite fields, Comm. Algebra 31 (2003), 39553982. MR 2007391. DOI 10.1081/AGB-120022449.

[Ko1] J. Kollár, Extremal rays on smooth threefolds, Ann. Sci. Éc. Norm. Supér. (4) 24 (1991), 339-361. MR 1100994.

[Ko2] - Rational Curves on Algebraic Varieties, Ergeb. Math. Grenzgeb. (3) 32, Springer, Berlin, 1996. MR 1440180. DOI 10.1007/978-3-662-03276-3.

[KoK] J. Kollár and S. Kovács, Birational geometry of log surfaces, preprint.

[KoM] J. Kollár and S. Mori, Birational Geometry of Algebraic Varieties, Cambridge Tracts in Math. 134, Cambridge University Press, Cambridge, 1998. MR 1658959. DOI 10.1017/CBO9780511662560.

[L] J. Lipman, Rational singularities, with applications to algebraic surfaces and unique factorization, Publ. Math. Inst. Hautes Études Sci. 36 (1969), 195-279. MR 0276239.

[M] V. Maşek, Kodaira-Iitaka and numerical dimensions of algebraic surfaces over the algebraic closure of a finite field, Rev. Roumaine Math. Pures Appl. 38 (1993), 679-685. MR 1263211.

[Ma] H. Matsumura, Commutative Algebra, Benjamin, New York, 1970. MR 0266911.

[Mi] M. Miyanishi, Noncomplete Algebraic Surfaces, Lecture Notes in Math. 857, Springer, Berlin, 1981. MR 0635930.

[Mo1] S. Mori, Projective manifolds with ample tangent bundles, Ann. of Math. (2) 110 (1979), 593-606. MR 0554387. DOI 10.2307/1971241.

[Mo2] , Threefolds whose canonical bundles are not numerically effective, Ann. of Math. (2) 116 (1982), 133-176. MR 0662120. DOI 10.2307/2007050.

[Mu1] D. Mumford, The topology of normal singularities of an algebraic surface and a criterion for simplicity, Publ. Math. Inst. Hautes Études Sci. 9 (1961), 5-22. MR 0153682.

[Mu2] , "Enriques' classification of surfaces in char $p$, I" in Global Analysis (Papers in Honor of K. Kodaira), Univ. Tokyo Press, Tokyo, 1969, 325-339. MR 0254053. 
[R] M. Raynaud, "Contre-exemple au 'vanishing theorem' en caractéristique $p>0$ " in C. P. Ramanujam-A Tribute, Tata Inst. Fund. Res. Stud. Math. 8, Springer, Berlin, 1978, 273-278. MR 0541027.

[S] F. Sakai, "Classification of normal surfaces" in Algebraic Geometry (Brunswick, Maine, 1985), Proc. Sympos. Pure Math. 46, Pt. 1, Amer. Math. Soc., Providence, 1987, 451-465. MR 0927967.

[T] H. Tanaka, The X-method for klt surfaces in positive characteristic, preprint, 2012.

[To] B. Totaro, Moving codimension-one subvarieties over finite fields, Amer. J. Math. 131 (2009), 1815-1833. MR 2567508. DOI 10.1353/ajm.0.0088.

Department of Mathematics

Graduate School of Science

Kobe University

Kobe 657-8501

Japan

tanakahi@math.kobe-u.ac.jp 